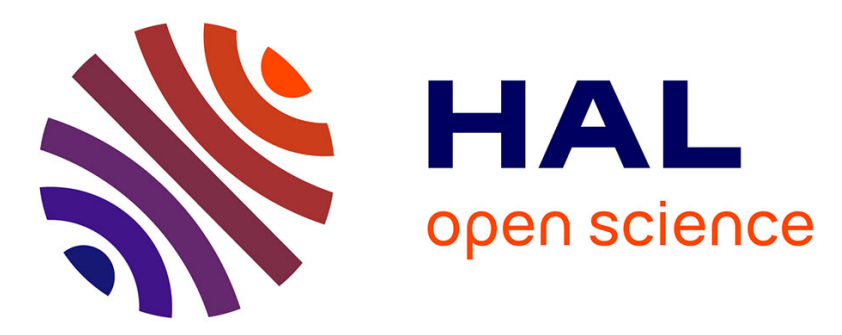

\title{
Generalized Impedance Boundary Conditions and Shape Derivatives for 3D Helmholtz Problems
}

Djalil Kateb, Frédérique Le Louër

\section{To cite this version:}

Djalil Kateb, Frédérique Le Louër. Generalized Impedance Boundary Conditions and Shape Derivatives for 3D Helmholtz Problems. Mathematical Models and Methods in Applied Sciences, 2016, 10.1142/S0218202516500500 . hal-01267024v2

\section{HAL Id: hal-01267024 \\ https://hal.science/hal-01267024v2}

Submitted on 11 Feb 2016

HAL is a multi-disciplinary open access archive for the deposit and dissemination of scientific research documents, whether they are published or not. The documents may come from teaching and research institutions in France or abroad, or from public or private research centers.
L'archive ouverte pluridisciplinaire HAL, est destinée au dépôt et à la diffusion de documents scientifiques de niveau recherche, publiés ou non, émanant des établissements d'enseignement et de recherche français ou étrangers, des laboratoires publics ou privés. 


\title{
Generalized Impedance Boundary Conditions and Shape Derivatives for 3D Helmholtz Problems
}

\author{
Djalil Kateb*1 and Frédérique Le Louër ${ }^{\dagger 1}$ \\ ${ }^{1}$ Sorbonne Universités, Université de technologie de Compiègne, LMAC EA2222 Laboratoire de Mathématiques \\ Appliquées de Compiègne - CS 60319 - 60203 Compiègne cedex, France
}

\begin{abstract}
This paper is concerned with the shape sensitivity analysis of the solution to the Helmholtz transmission problem for three dimensional sound-soft or sound-hard obstacles coated by a thin layer. This problem can be asymptotically approached by exterior problems with an improved condition on the exterior boundary of the coated obstacle, called Generalised Impedance Boundary Condition (GIBC). Using a series expansion of the Laplacian operator in the neighborhood of the exterior boundary, we retrieve the first order GIBCs characterizing the presence of an interior thin layer with either a constant or a variable thickness. The first shape derivative of the solution to the original Helmholtz transmission problem solves a new thin layer transmission problem with non vanishing jumps across the exterior and the interior boundary of the thin layer. In the special case of thin layers with a constant thickness, we show that we can interchange the first order differentiation with respect to the shape of the exterior boundary and the asymptotic approximation of the solution. Numerical experiments are presented to highlight the various theoretical results.
\end{abstract}

\section{Introduction}

This paper is devoted to the shape sensitivity analysis of the solution to time-harmonic acoustic scattering problems in the special case where the scattering object is a three-dimensional sound-soft or a sound-hard obstacle coated by a thin layer whose width $\varepsilon$ tends to zero. It is well known that the use of boundary and finite elements methods for solving this scattering problems fail since some numerical instabilities arise. Indeed, we face two kind of scalings : a big scale for the exterior of the obstacle and a very small one which corresponds to the thin layer. To avoid the phenomenon, we are led to approximate the original model by a new exterior boundary value problem with high order boundary conditions in terms of surface derivatives, called generalized impedance boundary conditions (GIBC). The exact solution is given through an asymptotic expansion in terms of the thickness parameter $\varepsilon$ where each coefficient function is the solution of a boundary value problem set on a geometry independent on $\varepsilon$. In practice, we are only interested by a finite number of terms in the asymptotic expansion. The GIBC satisfied by the approximate solution leads to an error estimate up to $O\left(\varepsilon^{N+1}\right)$, where $N$ is the order of truncation in the asymptotic expansion of the exact solution. These conditions have been first derived by Bendali and Lemrabet in [4] in the case of thin layer with a constant thickness and more recently they were generalised to the $2 \mathrm{D}$ case of thin layer with a variable thickness in 3 .

The work finds its motivation in the recent study of inverse scattering problems (see [7, 8, 9, 11]) or shape optimization problems (see [15]). The authors take the approximation of order 1 of the original problem and present a theoretical analysis based on the shape derivative of the approximate solution. Our natural question is the following : what happens if we compute first the shape derivative of the original problem (with the coated context) and then take the corresponding GIBC of order 1. The purpose of the paper is to give here a general result about the norm of the difference of the shape derivatives for an approximation of order $N$. We show that the error is up to $O\left(\varepsilon^{N+1}\right)$.

Let consider a simply connected bounded domain $\Omega$ in $\mathbb{R}^{3}$, with a closed orientable boundary $\Gamma$, as smooth as we need, representing a sound-soft or a sound-hard scatterrer $\Omega^{\varepsilon}$ coated by a thin layer denoted

*djalil.kateb@utc.fr

${ }^{\dagger}$ frederique.le-louer@utc.fr 
$\Omega_{i n t}^{\varepsilon}$. Let $\varepsilon>0$ and $h$ be a positive smooth function defined on $\Gamma$. The thin layer with variable thickness surrounding the acoustic object is defined by:

$$
\Omega_{\text {int }}^{\varepsilon}=\{\boldsymbol{x}+\delta h(\boldsymbol{x}) \boldsymbol{n}(\boldsymbol{x}) \mid \boldsymbol{x} \in \Gamma \text { and }-\varepsilon<\delta<0\} .
$$

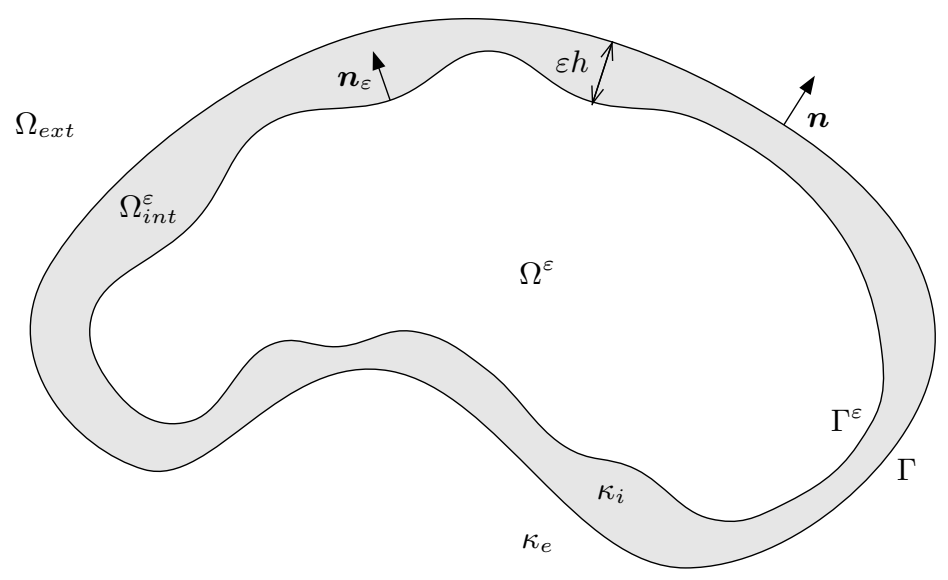

We set $\Gamma^{\varepsilon}=\partial \Omega^{\varepsilon}$ so that we have $\Omega=\Omega_{\text {int }}^{\varepsilon} \cup \Gamma^{\varepsilon} \cup \Omega^{\varepsilon}$. We denote by $\boldsymbol{n}$ and $\boldsymbol{n}_{\varepsilon}$ the outward unit normal vectors to $\Gamma$ and $\Gamma^{\varepsilon}$, respectively, and by $\Omega_{e x t}=\mathbb{R}^{3} \backslash \Omega$ the unbounded exterior domain. Throughout the paper we denote by $H^{t}\left(\Omega_{i n t}^{\varepsilon}\right), H_{l o c}^{t}\left(\overline{\Omega_{e x t}}\right)$ and $H^{t}(\Gamma)$ the standard (local in the case of the exterior domain) complex valued, Hilbertian Sobolev space of order $t \in \mathbb{R}$ defined on $\Omega_{i n t}^{\varepsilon}, \overline{\Omega_{e x t}}, \Gamma$ and $\Gamma^{\varepsilon}$ respectively (with the convention $H^{0}=L^{2}$.) The exterior wavenumber $\kappa_{e}$, the interior wavenumber $\kappa_{i}$ and the density ratio $\rho$ are given positive constants. We are concerned with the following transmission problem : Given any densities $f_{\text {ext }} \in H^{\frac{1}{2}}(\Gamma)$ and $g_{\text {ext }} \in H^{-\frac{1}{2}}(\Gamma)$, find the solution $\left(u_{\text {int }}^{\varepsilon}, u_{\text {ext }}^{\varepsilon}\right) \in H^{1}\left(\Omega_{\text {int }}^{\varepsilon}\right) \times H_{\text {loc }}^{1}\left(\Omega_{\text {ext }}\right)$ satisfying

$$
\left\{\begin{array}{cccl}
\Delta u_{i n t}^{\varepsilon}+\kappa_{i}^{2} u_{i n t}^{\varepsilon} & = & 0 & \text { in } \Omega_{i n t}^{\varepsilon} \\
\Delta u_{\text {ext }}^{\varepsilon}+\kappa_{e}^{2} u_{\text {ext }}^{\varepsilon} & = & 0 & \text { in } \Omega_{e x t} \\
u_{i n t}^{\varepsilon}-u_{\text {ext }}^{\varepsilon} & = & f_{\text {ext }} & \text { on } \Gamma \\
\rho \partial_{\boldsymbol{n}} u_{i n t}^{\varepsilon}-\partial_{\boldsymbol{n}} u_{\text {ext }}^{\varepsilon} & = & g_{\text {ext }} & \text { on } \Gamma
\end{array}\right.
$$

and either a Dirichlet boundary condition on $\Gamma^{\varepsilon}$

$$
u_{i n t}^{\varepsilon}=f_{i n t}^{\varepsilon},
$$

or a Neumann boundary condition on $\Gamma^{\varepsilon}$

$$
\partial_{\boldsymbol{n}_{\varepsilon}} u_{i n t}^{\varepsilon}=\boldsymbol{n}_{\varepsilon} \cdot\left(\nabla u_{i n t}^{\varepsilon}\right)_{\mid \Gamma^{\varepsilon}}=g_{i n t}^{\varepsilon} .
$$

To ensure the uniqueness of the solution to either the problem 1.1$]-1.2$ or $1.1-1.3$, the scattered field $u_{e x t}^{\varepsilon}$ is assumed to solve the Sommerfield radiation condition $\lim _{|\boldsymbol{x}| \rightarrow+\infty}|\boldsymbol{x}|\left|\partial_{\boldsymbol{n}} u(\boldsymbol{x})-i \kappa u(\boldsymbol{x})\right|=0$ uniformly in all directions $\boldsymbol{x} /|\boldsymbol{x}|$. Following the proof of Theorem 2.1 in [25], one can prove that the thin-layer transmission problem has at most one solution. Existence of a solution can be proved using boundary integral equation methods 25, 34]. More details can be found in the Appendix. The radiation condition implies that the scattered field $u_{e x t}$ has an asymptotic behavior of the form $u_{e x t}^{\varepsilon}(\boldsymbol{x})=\frac{e^{i \kappa|\boldsymbol{x}|}}{|\boldsymbol{x}|} u_{\infty}^{\varepsilon}(\hat{\boldsymbol{x}})+O\left(\frac{1}{|\boldsymbol{x}|}\right),|\boldsymbol{x}| \rightarrow \infty$, uniformly in all directions $\hat{\boldsymbol{x}}=\frac{\boldsymbol{x}}{|\boldsymbol{x}|}$. The far-field pattern $u_{\infty}^{\varepsilon}$ is a scalar function defined on the unit sphere $\mathbb{S}^{2}$ of $\mathbb{R}^{3}$ and is always analytic.

The scattering problem of time-harmonic waves by the coated obstacle $\Omega$ leads to special cases of the above transmission problems where the given densities $f_{\text {ext }}$ and $g_{\text {ext }}$ are the boundary data of an incident plane wave $u^{i n c}(\boldsymbol{x})=e^{i \kappa_{e} \boldsymbol{d} \cdot \boldsymbol{x}}, \boldsymbol{d} \in \mathbb{S}^{2}$. The total displacement field $u_{e x t}^{\varepsilon}+u^{i n c}$ is then given by the superposition of the incident field $u^{i n c}$, which is an entire solution of the Helmholtz equation, and the scattered field $u_{\text {ext }}^{\varepsilon}$, which solves the Helmholtz equation in $\Omega_{e x t}$ and the Sommerfield radiation condition. In this cases, we assume $f_{i n t}^{\varepsilon}=0$ and $g_{i n t}^{\varepsilon}=0$. In Section 3 , for small positive real values of $\varepsilon$, we approach the solution $u_{e x t}^{\varepsilon}$ of (1.1) by the solution $v_{[N]}^{\varepsilon}$ of some exterior boundary value problems of the form

$$
\left\{\begin{array}{clll}
\Delta v_{[N]}^{\varepsilon}+\kappa_{e}^{2} v_{[N]}^{\varepsilon} & = & 0 & \text { in } \Omega_{e x t} \\
C\left(\varepsilon, \partial_{\boldsymbol{n}}\left(v_{[N]}^{\varepsilon}+u^{i n c}\right),\left(v_{[N]}^{\varepsilon}+u^{i n c}\right)\right) & = & 0 & \text { on } \Gamma \\
\lim _{|\boldsymbol{x}| \rightarrow+\infty}|\boldsymbol{x}|\left|\partial_{\boldsymbol{n}} v_{[N]}^{\varepsilon}(\boldsymbol{x})-i \kappa v_{[N]}^{\varepsilon}(\boldsymbol{x})\right| & = & 0
\end{array}\right.
$$


where the linear condition on the boundary $C\left(\varepsilon, \partial_{\boldsymbol{n}}\left(v_{[N]}^{\varepsilon}+u^{i n c}\right),\left(v_{[N]}^{\varepsilon}+u^{i n c}\right)\right)=0$ is the so-called Generalised Impedance Boundary Condition (GIBC). To construct the GIBCs, we extend to $\mathbb{R}^{3}$ the approach developed in [3] to model the 2D case of thin layers with variable thickness. The technique originates from the PhD thesis 33 and is based on an asymptotic expansion of the Helmholtz equation in $\Omega_{i n t}^{\varepsilon}$ in terms of $\varepsilon$ and surface derivatives on $\Gamma$ presented in Section 2 We introduce a rapid variable $S$ and thanks to the transformation of the thin layer into a band of thickness $h$ we get $u_{i n t}^{\varepsilon}$ as a solution to a Sturm-Liouville type problem of variable $S$. Once the differential equation is solved, then thanks to boundary conditions and jump condition, we get the corresponding boundary condition corresponding to the exterior domain. The existence and uniqueness of a solution to these problems can be found in [12] for $N=1,2$. This approach leads first to estimate $\left\|u_{e x t}^{\varepsilon}-v_{[N]}^{\varepsilon}\right\|_{H^{\frac{1}{2}}(\Gamma)}=O\left(\varepsilon^{N+1}\right)$ and we deduce $\left\|u_{e x t}^{\varepsilon}-v_{[N]}^{\varepsilon}\right\|_{H^{1}\left(\Omega_{e x t} \cap B_{R}\right)}=O\left(\varepsilon^{N+1}\right)$ for every ball $B_{R}$ of radius $R$ and $\left\|u_{\infty}^{\varepsilon}-v_{\infty,[N]}^{\varepsilon}\right\|_{L^{2}\left(\mathbb{S}^{2}\right)}=O\left(\varepsilon^{N+1}\right)$ where $v_{\infty,[N]}^{\varepsilon}$ is the far-field pattern of the approximate solution $v_{[N]}^{\varepsilon}$.

Then, assuming the thin layer having a constant thickness, we analyze the dependence of the solution, or equivalently its far-field pattern, to the transmission problem 1.1 with respect to the shape of the exterior boundary $\Gamma$. The first shape derivative $\dot{u}_{\text {ext }}^{\varepsilon}$ solve the transmission problem (1.1) with non vanishing jumps accross the exterior and the interior boundaries. On one hand, the shape derivative $\dot{u}_{\text {ext }}^{\varepsilon}$ is approached in Section 4 by the solution $w_{[N]}^{\varepsilon}$ of some exterior boundary value problems of the form

$$
\left\{\begin{array}{clll}
\Delta w_{[N]}^{\varepsilon}+\kappa_{e}^{2} w_{[N]}^{\varepsilon} & = & 0 & \text { in } \Omega_{e x t} \\
C\left(\varepsilon, \partial_{\boldsymbol{n}} w_{[N]}^{\varepsilon}, w_{[N]}^{\varepsilon}\right) & = & F_{[N]}^{\varepsilon, 1} \quad \text { on } \Gamma \\
\lim _{|\boldsymbol{x}| \rightarrow+\infty}|\boldsymbol{x}|\left|\partial_{\boldsymbol{n}} w_{[N]}^{\varepsilon}(\boldsymbol{x})-i \kappa w_{[N]}^{\varepsilon}(\boldsymbol{x})\right| & = & 0,
\end{array}\right.
$$

where the right-hand side $F_{[N]}^{\varepsilon, 1}$ can be expressed in terms of the boundary data of the exterior total field $v_{[N]}^{\varepsilon}+u^{i n c}$. In this case we naturally obtain $\left\|\dot{u}_{\infty}^{\varepsilon}-w_{\infty,[N]}^{\varepsilon}\right\|_{L^{2}\left(\mathbb{S}^{2}\right)}=O\left(\varepsilon^{N+1}\right)$ where $w_{\infty,[N]}^{\varepsilon}$ is the far-field pattern of the approximate derivative $w_{[N]}^{\varepsilon}$. On the other hand, we provide in Section 5 the characterisation of the first shape derivative $\dot{v}_{[N]}^{\varepsilon}$ of the solution $v_{[N]}^{\varepsilon}$ to the exterior problem 1.4 of the form

$$
\left\{\begin{array}{clll}
\Delta \dot{v}_{[N]}^{\varepsilon}+\kappa_{e}^{2} \dot{v}_{[N]}^{\varepsilon} & = & 0 & \text { in } \Omega_{e x t} \\
C\left(\varepsilon, \partial_{\boldsymbol{n}} \dot{v}_{[N]}^{\varepsilon}, \dot{v}_{[N]}^{\varepsilon}\right) & = & F_{[N]}^{\varepsilon, 2} & \text { on } \Gamma \\
\lim _{|\boldsymbol{x}| \rightarrow+\infty}|\boldsymbol{x}|\left|\partial_{\boldsymbol{n}} \dot{v}_{[N]}^{\varepsilon}(\boldsymbol{x})-i \kappa \dot{v}_{[N]}^{\varepsilon}(\boldsymbol{x})\right| & = & 0,
\end{array}\right.
$$

where the right-hand side $F_{[N]}^{\varepsilon, 2}$ can be expressed in terms of the boundary data of the exterior total field $v_{[N]}^{\varepsilon}+u^{i n c}$. In Section 6, we prove for $N=0,1,2$ that the two approaches are equivalent, which means $\left\|\dot{v}_{[N]}^{\varepsilon}-w_{[N]}^{\varepsilon}\right\|_{H^{\frac{1}{2}(\Gamma)}}=O\left(\varepsilon^{N+1}\right)$ and $\left\|\dot{v}_{\infty,[N]}^{\varepsilon}-w_{\infty,[N]}^{\varepsilon}\right\|_{L^{2}\left(\mathbb{S}^{2}\right)}=O\left(\varepsilon^{N+1}\right)$ where $\dot{v}_{\infty,[N]}^{\varepsilon}$ is the far-field pattern of the derivative $\dot{v}_{[N]}^{\varepsilon}$. The various theoretical results are illustrated by some numerical experiments in Section 7. The transmission problem and the exterior boundary value problems are solved using boundary integral equation methods [13, 34, (see the Appendix) and the high order spectral method [18. Finally, we draw concluding remarks and we discuss possible research lines in Section 8

\section{Elementary differential geometry and asymptotic expansions}

In this section, we derive the asymptotic expansion of the Laplacian operator in the neighborhood of $\Gamma$ using the high-order material derivatives of some surface differential operators and Taylor-Young expansions. We use the surface differential operators: The tangential gradient $\nabla_{\Gamma}$, the surface divergence $\operatorname{div}_{\Gamma}$ and the scalar Laplace-Beltrami operator $\Delta_{\Gamma}$. For their definitions we refer to Nedelec's book [27] (pp. 68-75). We use the notations of [27] and quote some usefull results from [27] (pp. 67-78) and [1].

Since $\Gamma$ is a smooth closed orientable boundary, there exists a tubular neighbourhood ${\stackrel{\circ}{S_{0}}}_{s_{0}}$ of $\Gamma$ in which any point $\boldsymbol{y}$ admits the unique expansion

$$
\boldsymbol{y}=\boldsymbol{x}+\operatorname{sn}(\boldsymbol{x}) \text {, with } \boldsymbol{x} \in \Gamma \text {, and } s \in]-s_{0} ; s_{0}\left[\text { with } s_{0}>0 .\right.
$$

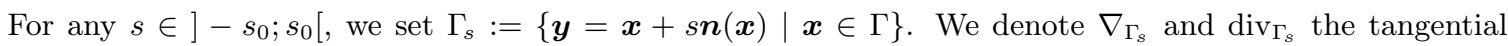
gradient and the surface divergence on $\Gamma_{s}$, respectively, and we denote by $\boldsymbol{n}_{s}$ the outward unit normal vector to $\Gamma_{s}$. For any scalar function $u$ and vector function $\boldsymbol{w}$ defined in ${\stackrel{\circ}{\Gamma_{s}}}_{s_{0}}$, the following expansions hold on $\Gamma_{s}$ :

$$
\nabla u=\nabla_{\Gamma_{s}} u+\boldsymbol{n}_{s} \partial_{s} u,
$$


and

$$
\operatorname{div} \boldsymbol{w}=\operatorname{div}_{\Gamma_{s}} \boldsymbol{w}+\left(\boldsymbol{n}_{s} \cdot \partial_{s} \boldsymbol{w}\right) .
$$

We denote by $\tau_{s}$ the transformation that maps the restriction $u_{\mid \Gamma_{s}}$ of $u$ to $\Gamma_{s}$ to the function defined on $\Gamma$ by $\left(\tau_{s} u_{\mid \Gamma_{s}}\right)(\boldsymbol{x})=u_{\mid \Gamma_{s}}(\boldsymbol{x}+s \boldsymbol{n}(\boldsymbol{x}))$. Setting $\left(\tau_{s} u_{\mid \Gamma_{s}}\right)(\boldsymbol{x})=\bar{u}(\boldsymbol{x}, s)$, we define an isomorphism between ${\stackrel{\circ}{\Gamma_{s}}}_{s_{0}}$ and $\Gamma \times]-s_{0} ; s_{0}\left[\right.$. The outward unit normal vector $\boldsymbol{n}_{s}$ to the boundary $\Gamma_{s}$ satisfies $\boldsymbol{n}_{s}=\tau_{s}^{-1} \boldsymbol{n}$. Using this change of coordinate system we can write for $\boldsymbol{y} \in \Gamma_{s}$ :

$$
(\nabla u)(\boldsymbol{y})=\left(\tau_{s} \nabla u\right)(\boldsymbol{x})=\tau_{s} \nabla_{\Gamma_{s}} \tau_{s}^{-1} \bar{u}(\boldsymbol{x}, s)+\boldsymbol{n} \partial_{s} \bar{u}(\boldsymbol{x}, s),
$$

and

$$
(\operatorname{div} \boldsymbol{w})(\boldsymbol{y})=\tau_{s}(\operatorname{div} \boldsymbol{w})(\boldsymbol{x})=\tau_{s} \operatorname{div}_{\Gamma_{s}} \tau_{s}^{-1} \overline{\boldsymbol{w}}(\boldsymbol{x}, s)+\boldsymbol{n} \cdot \partial_{s} \overline{\boldsymbol{w}}(\boldsymbol{x}, s) .
$$

The material derivatives of the surface differential operators has been analysed in [14, Section 5] and we obtain the following result.

Proposition 2.1. The functions defined by $s \in]-s_{0} ; s_{0}\left[\mapsto \tau_{s} \nabla_{\Gamma_{s}} \tau_{s}^{-1} \in \mathscr{L}\left(\mathscr{C}^{1}(\Gamma), \mathscr{C}^{0}\left(\Gamma, \mathbb{R}^{3}\right)\right)\right.$ and $s \in$ ]$-s_{0} ; s_{0}\left[\mapsto \tau_{s} \operatorname{div}_{\Gamma_{s}} \tau_{s}^{-1} \in \mathscr{L}\left(\mathscr{C}^{1}\left(\Gamma, \mathbb{R}^{3}\right), \mathscr{C}^{0}(\Gamma)\right)\right.$ are infinitely differentiable and we have for any $u_{0} \in \mathscr{C}^{1}(\Gamma)$ and $\boldsymbol{w}_{0} \in \mathscr{C}^{1}\left(\Gamma, \mathbb{R}^{3}\right)$ :

$$
\partial_{s}\left(\tau_{s} \nabla_{\Gamma_{s}} \tau_{s}^{-1}\right) u_{0}=-\tau_{s} \mathcal{R}_{s} \nabla_{\Gamma_{s}} \tau_{s}^{-1} u_{0}
$$

and

$$
\partial_{s}\left(\tau_{s} \operatorname{div}_{\Gamma_{s}} \tau_{s}^{-1}\right) \boldsymbol{w}_{0}=-\tau_{s} \operatorname{div}_{\Gamma_{s}} \mathcal{R}_{s} \tau_{s}^{-1} \boldsymbol{w}_{0}+\tau_{s} \nabla_{\Gamma_{s}} \mathcal{H}_{s} \cdot \boldsymbol{w}_{0}-\operatorname{Trace}\left[\tau_{s} \mathcal{R}_{s}^{2}\right]\left(\boldsymbol{n} \cdot \boldsymbol{w}_{0}\right)
$$

where $\mathcal{R}_{s}=\nabla_{\Gamma_{s}} \boldsymbol{n}$ and $\mathcal{H}_{s}=\operatorname{Trace}\left[\mathcal{R}_{s}\right]$.

The first order material derivatives corresponds to the commutators given in 27. Eqs. (2.5.228) and (2.5.229)]. To obtain the high order derivatives, it suffices to use the chain rule since we have [27, Eq $(2.5 .154)$ and $(2.5 .155)]$

$$
\partial_{s}\left(\tau_{s} \mathcal{R}_{s}\right)=-\tau_{s} \mathcal{R}_{s}^{2} \text { and } \partial_{s}\left(\tau_{s} \mathcal{H}_{s}\right)=-\operatorname{Trace}\left[\tau_{s} \mathcal{R}_{s}^{2}\right]
$$

Further, we will use the gaussian curvature denoted by $\mathcal{G}_{s}$ which satisfies

$$
\operatorname{Trace}\left[\mathcal{R}_{s}^{2}\right]+2 \mathcal{G}_{s}=\mathcal{H}_{s}^{2} .
$$

and if we set $\Pi_{3}=\mathrm{I}_{3}-\boldsymbol{n} \otimes \boldsymbol{n}$, then the Cayley Hamilton's theorem implies

$$
\mathcal{R}_{s}^{2}-\mathcal{H}_{s} \mathcal{R}_{s}+\mathcal{G}_{s} \Pi_{3}=0
$$

Using the Taylor-Young formula in the neighbourhood of $s=0$ and 2.1, we can expand the gradient operator in the coordinate system $(\boldsymbol{x}, s) \in \Gamma \times]-s_{0} ; s_{0}[$ and we obtain for any $N \in \mathbb{N}$

$$
\begin{aligned}
(\nabla u)(\boldsymbol{x}+s \boldsymbol{n}(\boldsymbol{x}))= & \boldsymbol{n}(\boldsymbol{x}) \partial_{s} \bar{u}(\boldsymbol{x}, s)+\nabla_{\Gamma} \bar{u}(\boldsymbol{x}, s) \\
& +\sum_{\ell=0}^{N} s^{\ell} \frac{1}{\ell !} \partial_{s}^{\ell}\left(\tau_{s} \nabla_{\Gamma_{s}} \tau_{s}^{-1}\right)_{\mid s=0} \bar{u}(\boldsymbol{x}, s)+O\left(s^{N+1}\right),
\end{aligned}
$$

with $\frac{1}{\ell !} \partial_{s}^{\ell}\left(\tau_{s} \nabla_{\Gamma_{s}} \tau_{s}^{-1}\right)_{\mid s=0}=(-1)^{\ell} \mathcal{R}^{\ell} \nabla_{\Gamma}$. In the same way, we write

$$
\begin{aligned}
(\operatorname{div} \boldsymbol{w})(\boldsymbol{x}+s \boldsymbol{n}(\boldsymbol{x}))= & \boldsymbol{n}(\boldsymbol{x}) \cdot \partial_{s} \overline{\boldsymbol{w}}(\boldsymbol{x}, s)+\operatorname{div}_{\Gamma} \overline{\boldsymbol{w}}(\boldsymbol{x}, s) \\
& +\sum_{\ell=1}^{N} s^{\ell} \frac{1}{\ell !} \partial_{s}^{\ell}\left(\tau_{s} \operatorname{div}_{\Gamma_{s}} \tau_{s}^{-1}\right)_{\mid s=0} \overline{\boldsymbol{w}}(\boldsymbol{x}, s)+O\left(s^{N+1}\right),
\end{aligned}
$$

with

$$
\begin{aligned}
\partial_{s}\left(\tau_{s} \operatorname{div}_{\Gamma_{s}} \tau_{s}^{-1}\right)_{\mid s=0} \overline{\boldsymbol{w}} & =-\operatorname{div}_{\Gamma} \mathcal{R} \overline{\boldsymbol{w}}+\nabla_{\Gamma} \mathcal{H} \cdot \overline{\boldsymbol{w}}-\operatorname{Trace}\left[\mathcal{R}^{2}\right](\boldsymbol{n} \cdot \overline{\boldsymbol{w}}) \\
& =-\left(\operatorname{div}_{\Gamma}(\mathcal{R}-\mathcal{H}) \Pi_{3} \overline{\boldsymbol{w}}+\mathcal{H} \operatorname{div}_{\Gamma} \Pi_{3} \overline{\boldsymbol{w}}+\left(\mathcal{H}^{2}-2 \mathcal{G}\right)(\boldsymbol{n} \cdot \overline{\boldsymbol{w}})\right),
\end{aligned}
$$

where $\Pi_{3}=\mathrm{I}_{3}-\boldsymbol{n} \otimes \boldsymbol{n}$ and using the chain rules we obtain the following high order terms

$$
\begin{aligned}
\frac{1}{2 !} \partial_{s}^{2}\left(\tau_{s} \operatorname{div}_{\Gamma_{s}} \tau_{s}^{-1}\right)_{\mid s=0} \overline{\boldsymbol{w}}= & \mathcal{H} \operatorname{div}_{\Gamma}(\mathcal{R}-\mathcal{H}) \Pi_{3} \overline{\boldsymbol{w}}+\left(\mathcal{H}^{2}-\mathcal{G}\right) \operatorname{div}_{\Gamma} \Pi_{3} \overline{\boldsymbol{w}} \\
& +\left(\mathcal{H}^{3}-3 \mathcal{H} \mathcal{G}\right)(\boldsymbol{n} \cdot \overline{\boldsymbol{w}})
\end{aligned}
$$

and

$$
\begin{aligned}
\frac{1}{3 !} \partial_{s}^{3}\left(\tau_{s} \operatorname{div}_{\Gamma_{s}} \tau_{s}^{-1}\right)_{\mid s=0} \overline{\boldsymbol{w}}= & -\left[\left(\mathcal{H}^{2}-\mathcal{G}\right) \operatorname{div}_{\Gamma}(\mathcal{R}-\mathcal{H}) \Pi_{3} \overline{\boldsymbol{w}}+\left(\mathcal{H}^{3}-2 \mathcal{G H}\right) \operatorname{div}_{\Gamma} \Pi_{3} \overline{\boldsymbol{w}}\right] \\
& -\operatorname{Trace}\left[\mathcal{R}^{4}\right](\boldsymbol{n} \cdot \overline{\boldsymbol{w}})
\end{aligned}
$$


The formula 2.5 is in accordance with the formula (2.5.182) in 27]

$$
\tau_{s} \nabla_{\Gamma_{s}} \tau_{s}^{-1} \bar{u}(\boldsymbol{x}, s)=(\mathrm{I}+s \mathcal{R}(\boldsymbol{x}))^{-1} \nabla_{\Gamma} \bar{u}(\boldsymbol{x}, s) .
$$

Indeed, the Neumann series of $(\mathrm{I}+s \mathcal{R}(\boldsymbol{x}))^{-1}$ yields the infinite series given in 2.5 . We deduce that the gradient operator is equal to its Taylor series in the tubular $\stackrel{\circ}{\Gamma}_{s_{0}}$. Since we have $\operatorname{div} \boldsymbol{w}=\operatorname{Trace}[\nabla \boldsymbol{w}]$, we also deduce that the divergence operator is equal to its Taylor series in $\stackrel{\circ}{\Gamma}_{s_{0}}$. However, the high-order terms are easier to obtain by computing the material derivatives than taking the trace of $\left[\mathcal{R}^{\ell} \nabla_{\Gamma} \boldsymbol{w}\right]$ for any $\ell \in \mathbb{N}$.

Assuming $\forall x \in \Gamma, 0<\varepsilon h(\boldsymbol{x})<s_{0}$, then we use the change of variable $s=-\varepsilon S$ with $S \in[0 ; h(\boldsymbol{x})]$. We set $\bar{u}(\boldsymbol{x}, s)=\bar{u}(\boldsymbol{x},-\varepsilon S)=\mathbf{U}(\boldsymbol{x}, S)$ and we have

$$
\partial_{s} \bar{u}(\boldsymbol{x}, s)=-\frac{1}{\varepsilon} \partial_{S} \mathbf{U}(\boldsymbol{x}, S) .
$$

Combining 2.5 and $(2.6)$, we obtain the asymptotic expansion of the Laplacian $\Delta=\operatorname{div} \nabla$

$$
\Delta=\frac{1}{\varepsilon^{2}}\left(\partial_{S}^{2}+\sum_{\ell=1}^{N} \varepsilon^{\ell} \Lambda_{\ell}+O\left(\varepsilon^{N+1}\right)\right)
$$

where

$$
\begin{gathered}
\Lambda_{1}=-\mathcal{H} \partial_{S}, \Lambda_{2}=\Delta_{\Gamma}-S\left(\mathcal{H}^{2}-2 \mathcal{G}\right) \partial_{S} \\
\Lambda_{3}=S\left(\operatorname{div}_{\Gamma}(2 \mathcal{R}-\mathcal{H}) \nabla_{\Gamma}+\mathcal{H} \Delta_{\Gamma}\right)-S^{2}\left(\mathcal{H}^{3}-3 \mathcal{H G}\right) \partial_{S}, \\
\Lambda_{4}=S^{2}\left(\operatorname{div}_{\Gamma}\left(2 \mathcal{R}^{2}-\mathcal{H} \mathcal{R}\right) \nabla_{\Gamma}+\mathcal{H} \operatorname{div}_{\Gamma}(2 \mathcal{R}-\mathcal{H}) \nabla_{\Gamma}+\left(\mathcal{H}^{2}-\mathcal{G}\right) \Delta_{\Gamma}\right)-S^{3} \operatorname{Trace}\left[\mathcal{R}^{4}\right] \partial_{S} .
\end{gathered}
$$

The following proposition gives an expression of the outward unit normal vector to the interior boundary $\Gamma^{\varepsilon}=\{\boldsymbol{y}=\boldsymbol{x}-\varepsilon h(\boldsymbol{x}) \boldsymbol{n}(\boldsymbol{x}) \mid \boldsymbol{x} \in \Gamma\}$ for any function $h$.

Proposition 2.2. The outward unit normal vector to $\Gamma^{\varepsilon}$ is given by

$$
\boldsymbol{n}_{\varepsilon}=\frac{\left(1-\varepsilon h \mathcal{H}+\varepsilon^{2} h^{2} \mathcal{G}\right) \boldsymbol{n}+\varepsilon\left(\mathrm{I}_{3}+\varepsilon h(\mathcal{R}-\mathcal{H})\right) \nabla_{\Gamma} h}{\sqrt{\left(1-\varepsilon h \mathcal{H}+\varepsilon^{2} h^{2} \mathcal{G}\right)^{2}+\varepsilon^{2}\left\|\left(\mathrm{I}_{3}+\varepsilon h(\mathcal{R}-\mathcal{H})\right) \nabla_{\Gamma} h\right\|^{2}}} .
$$

Proof. Assume that the tangent plane to $\Gamma$ at the point $\boldsymbol{x}$ is generated by the unit vectors $\boldsymbol{e}_{1}(\boldsymbol{x})$ and $\boldsymbol{e}_{2}(\boldsymbol{x})$ such that the outward unit normal vector to $\Gamma$ is defined by $\boldsymbol{n}=\boldsymbol{e}_{1} \times \boldsymbol{e}_{2}$. The cotangent vectors are given by $\boldsymbol{e}^{1}=\boldsymbol{e}_{2} \times \boldsymbol{n}$ and $\boldsymbol{e}^{2}=\boldsymbol{n} \times \boldsymbol{e}_{1}$. We have $\boldsymbol{e}_{i} \cdot \boldsymbol{e}^{j}=\delta_{i}^{j}$ where $\delta_{i}^{j}$ is the kronecker symbol. The tangent plane to $\Gamma^{\varepsilon}$ at the point $\boldsymbol{y}=\boldsymbol{x}-\varepsilon h(\boldsymbol{x}) \boldsymbol{n}(\boldsymbol{x})$ is generated by the vectors $\boldsymbol{e}_{1}(\boldsymbol{x}, \varepsilon)=\mathrm{D}[\mathrm{I}-\varepsilon h(\boldsymbol{x}) \boldsymbol{n}(\boldsymbol{x})] \boldsymbol{e}_{1}(\boldsymbol{x})$ and $\mathrm{D}[\mathrm{I}-\varepsilon h(\boldsymbol{x}) \boldsymbol{n}(\boldsymbol{x})] \boldsymbol{e}_{2}(\boldsymbol{x})$ and the outward unit normal vector to $\Gamma^{\varepsilon}$ is given by

$$
\boldsymbol{n}_{\varepsilon}(\boldsymbol{y})=\frac{\boldsymbol{e}_{1}(\boldsymbol{x}, \varepsilon) \times \boldsymbol{e}_{2}(\boldsymbol{x}, \varepsilon)}{\left\|\boldsymbol{e}_{1}(\boldsymbol{x}, \varepsilon) \times \boldsymbol{e}_{2}(\boldsymbol{x}, \varepsilon)\right\|} .
$$

It remains to compute $N_{h}^{\varepsilon}(\boldsymbol{x})=\boldsymbol{e}_{1}(\boldsymbol{x}, \varepsilon) \times \boldsymbol{e}_{2}(\boldsymbol{x}, \varepsilon)$. We have

$$
\begin{aligned}
N_{h}^{\varepsilon}= & \boldsymbol{e}_{1} \times \boldsymbol{e}_{2}-\varepsilon\left([\mathrm{D}(h \boldsymbol{n})] \boldsymbol{e}_{1} \times \boldsymbol{e}_{2}+\boldsymbol{e}_{1} \times[\mathrm{D}(h \boldsymbol{n})] \boldsymbol{e}_{2}\right)+\varepsilon^{2}\left([\mathrm{D}(h \boldsymbol{n})] \boldsymbol{e}_{1} \times[\mathrm{D}(h \boldsymbol{n})] \boldsymbol{e}_{2}\right) \\
= & \boldsymbol{n}-\varepsilon h\left(\mathcal{R} \boldsymbol{e}_{1} \times \boldsymbol{e}_{2}+\boldsymbol{e}_{1} \times \mathcal{R} \boldsymbol{e}_{2}\right)-\varepsilon\left(\left(\nabla_{\Gamma} h \cdot \boldsymbol{e}_{1}\right) \boldsymbol{n} \times \boldsymbol{e}_{2}+\left(\nabla_{\Gamma} h \cdot \boldsymbol{e}_{2}\right) \boldsymbol{e}_{1} \times \boldsymbol{n}\right) \\
& +\varepsilon^{2} h^{2} \mathcal{R} \boldsymbol{e}_{1} \times \mathcal{R} \boldsymbol{e}_{2}+\varepsilon^{2} h\left(\left(\nabla_{\Gamma} h \cdot \boldsymbol{e}_{1}\right) \boldsymbol{n} \times \mathcal{R} \boldsymbol{e}_{2}+\left(\nabla_{\Gamma} h \cdot \boldsymbol{e}_{2}\right) \mathcal{R} \boldsymbol{e}_{1} \times \boldsymbol{n}\right) .
\end{aligned}
$$

To conclude we use the following equalities

$$
\begin{gathered}
\mathcal{R} \boldsymbol{e}_{1} \times \boldsymbol{e}_{2}+\boldsymbol{e}_{1} \times \mathcal{R} \boldsymbol{e}_{2}=(\mathcal{H}-\mathcal{R}) \boldsymbol{e}_{1} \times \boldsymbol{e}_{2}=(\mathcal{H}-\mathcal{R}) \boldsymbol{n}=\mathcal{H} \boldsymbol{n}, \\
\left(\nabla_{\Gamma} h \cdot \boldsymbol{e}_{1}\right) \boldsymbol{n} \times \boldsymbol{e}_{2}+\left(\nabla_{\Gamma} h \cdot \boldsymbol{e}_{2}\right) \boldsymbol{e}_{1} \times \boldsymbol{n}=-\left(\nabla_{\Gamma} h \cdot \boldsymbol{e}_{1}\right) \boldsymbol{e}^{1}-\left(\nabla_{\Gamma} h \cdot \boldsymbol{e}_{2}\right) \boldsymbol{e}^{2}=-\nabla_{\Gamma} h, \\
\mathcal{R} \boldsymbol{e}_{1} \times \mathcal{R} \boldsymbol{e}_{2}=\operatorname{cof}[\mathcal{R}]\left(\boldsymbol{e}_{1} \times \boldsymbol{e}_{2}\right)=\mathcal{G} \boldsymbol{n}, \\
\boldsymbol{n} \times \mathcal{R} \boldsymbol{e}_{2}=\boldsymbol{n} \times \mathcal{R} \boldsymbol{e}_{2}+\mathcal{R} \boldsymbol{n} \times \boldsymbol{e}_{2}=-(\mathcal{H}-\mathcal{R}) \boldsymbol{e}^{1}, \\
\mathcal{R} \boldsymbol{e}_{1} \times \boldsymbol{n}=\mathcal{R} \boldsymbol{e}_{1} \times \boldsymbol{n}+\boldsymbol{e}_{1} \times \mathcal{R} \boldsymbol{n}=-(\mathcal{H}-\mathcal{R}) \boldsymbol{e}^{2} .
\end{gathered}
$$




\section{Construction of the GIBCs}

The construction of the GIBC is based on the assumption that the interior and exterior fields admits the following expansion when $\varepsilon$ tends zero :

$$
\begin{aligned}
u_{i n t}^{\varepsilon}(\boldsymbol{y})=\mathbf{U}_{i n t}^{\varepsilon}(\boldsymbol{x}, S) & =\sum_{\ell \geq 0} \varepsilon^{n} \mathbf{U}_{i n t}^{\ell}(\boldsymbol{x}, S) \text { in } \Gamma \times[0, h(\boldsymbol{x})], \\
u_{e x t}^{\varepsilon}(\boldsymbol{y}) & =\sum_{\ell \geq 0} \varepsilon^{n} u_{e x t}^{\ell}(\boldsymbol{y}) \text { in } \Omega_{e x t} .
\end{aligned}
$$

The problem 1.1 can be rewritten as follows:

$$
\left\{\begin{aligned}
\sum_{\ell \geq 0} \varepsilon^{\ell}\left(\Delta u_{e x t}^{\ell}+\kappa_{e}^{2} u_{e x t}^{\ell}\right) & = & & \text { in } \Omega_{e x t} \\
\sum_{\ell \geq 0} \varepsilon^{\ell} \partial_{S}^{2} \mathbf{U}_{i n t}^{\ell} & = & -\sum_{\ell \geq 1} \varepsilon^{\ell} \Lambda_{1} \mathbf{U}_{i n t}^{\ell-1}-\sum_{\ell \geq 2} \varepsilon^{\ell}\left(\Lambda_{2}+\kappa_{i}^{2} h^{2} \mathrm{I}\right) \mathbf{U}_{i n t}^{\ell-2} & \\
& -\sum_{k \geq 3} \sum_{\ell \geq k} \varepsilon^{\ell} \Lambda_{\ell} \mathbf{U}_{i n t}^{\ell-k} & & \text { in } \Gamma \times(0, h) \\
\sum_{\ell \geq 0} \varepsilon^{n} \mathbf{U}_{i n t}^{\ell}(\boldsymbol{x}, 0) & = & u^{i n c}(\boldsymbol{x})+\sum_{\ell \geq 0} \varepsilon^{\ell} u_{e x t}^{\ell}(\boldsymbol{x}) & \text { on } \Gamma \times\{0\} \\
\sum_{\ell \geq 0} \varepsilon^{\ell} \partial_{S} \mathbf{U}_{i n t}^{\ell}(\boldsymbol{x}, 0) & =-\frac{1}{\rho}\left(\varepsilon \partial_{\boldsymbol{n}} u^{i n c}(\boldsymbol{x})+\sum_{\ell \geq 1} \varepsilon^{\ell} \partial_{\boldsymbol{n}} u_{e x t}^{\ell-1}(\boldsymbol{x})\right) & & \text { on } \Gamma \times\{0\},
\end{aligned}\right.
$$

and the interior field satisfies either a Dirichlet boundary condition on $\Gamma \times\{h\}$

$$
\sum_{\ell \geq 0} \varepsilon^{\ell} \mathbf{U}_{i n t}^{\ell}=0
$$

or a Neumann boundary condition on $\Gamma \times\{h\}$ that can be rewritten using Proposition 2.2 as follows

$$
\begin{aligned}
\sum_{\ell \geq 0} \varepsilon^{\ell} \partial_{S} \mathbf{U}_{i n t}^{\ell}= & h \mathcal{H} \sum_{\ell \geq 1} \varepsilon^{\ell} \partial_{S} \mathbf{U}_{i n t}^{\ell-1} \\
& -h^{2} \mathcal{G} \sum_{\ell \geq 2} \varepsilon^{\ell} \partial_{S} \mathbf{U}_{i n t}^{\ell-2}+\sum_{\ell \geq 2} \varepsilon^{\ell} \nabla_{\Gamma} h \cdot \nabla_{\Gamma} \mathbf{U}_{i n t}^{\ell-2} \\
& +\sum_{k \geq 3} h^{k-2} \sum_{\ell \geq k} \varepsilon^{\ell} \nabla_{\Gamma} h \cdot(2 \mathcal{R}-\mathcal{H}) \mathcal{R}^{k-3} \nabla_{\Gamma} \mathbf{U}_{i n t}^{\ell-k}
\end{aligned}
$$

We identify the right and left hand sides of each equations in 3.1) according to the power $\ell \geq 0$ of $\varepsilon$ and we solve iteratively the new systems - that can be split into two systems of unknowns $\mathbf{U}_{i n t}^{\ell}$ and $u_{\text {ext }}^{\bar{\ell}}$ respectively - to compute first $\mathbf{U}_{i n t}^{\ell}$ and then recover the boundary condition satisfied by $u_{\text {ext }}^{\ell}$. From these results we deduce the GIBC satisfied by $v_{[N]}^{\varepsilon}$, which is an approximation of $\sum_{\ell=0}^{N} \varepsilon^{\ell} u_{e x t}^{\ell}$ up to $O\left(\varepsilon^{N+1}\right)$. The final results are stated in the following two propositions. We obtain similar results than in the $2 \mathrm{D}$ case 3 .

Proposition 3.1. The GIBCs modeling sound-soft obstacles coated by thin layers with a variable thickness are given for $N=0,1,2,3$ by

$$
\left(v_{[N]}^{\varepsilon h}+u^{i n c}\right)+B^{\varepsilon h, N} \partial_{\boldsymbol{n}}\left(v_{[N]}^{\varepsilon h}+u^{i n c}\right)=0
$$

where

$$
\begin{aligned}
B^{\varepsilon h, 0} & =0, \quad B^{\varepsilon h, 1}=-\frac{1}{\rho}(\varepsilon h) \mathrm{I}, \quad B^{\varepsilon h, 2}=-\frac{1}{\rho}(\varepsilon h)\left(1+\frac{(\varepsilon h)}{2} \mathcal{H}\right) \mathrm{I} \quad \text { and } \\
B^{\varepsilon h, 3} & =-\frac{1}{\rho} \varepsilon h\left(\mathrm{I}+\frac{\varepsilon h}{2} \mathcal{H} \mathrm{I}-\frac{(\varepsilon h)^{2}}{6} \Delta_{\Gamma}+\left[\frac{\varepsilon h}{2} \Delta_{\Gamma}+\frac{\varepsilon h}{3}\left(\kappa_{i}^{2}+\mathcal{H}^{2}-\mathcal{G}\right) \mathrm{I}\right] \varepsilon h\right) .
\end{aligned}
$$

Proof. Collecting the equations when $\ell=0$, we obtain the two systems

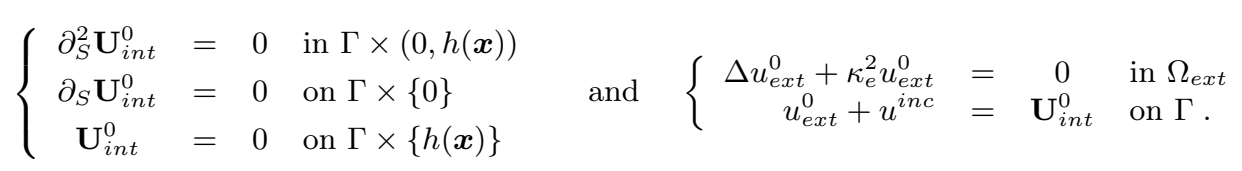


The first equation implies that $\mathbf{U}_{i n t}^{0}(\boldsymbol{x}, S)$ is a polynomial function of degre 1 in the variable $S$. The second equation implies that the leading coefficient is 0 and the third equation gives the constant term. We conclude

$$
\mathbf{U}_{\text {int }}^{0}(\boldsymbol{x}, S)=0 \text {. }
$$

In this case we approach $u_{e x t}^{\varepsilon}$ by the function $v_{[0]}^{\varepsilon}=u_{0}^{e x t}$ and then $u_{e x t}^{\varepsilon}-v_{[0]}^{\varepsilon}=O(\varepsilon)$. When $\ell=1$, we obtain the two systems

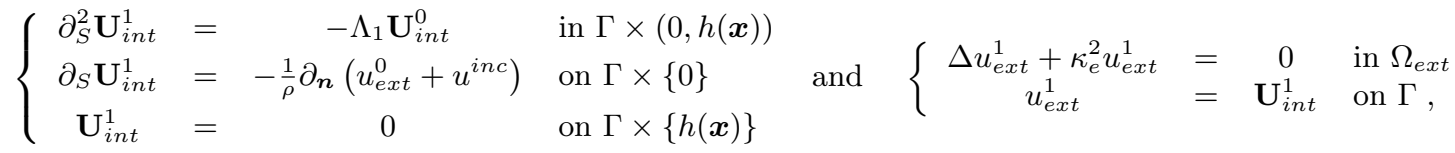

We conclude with similar arguments that

$$
\mathbf{U}_{i n t}^{1}(\cdot, S)=-(S-h(\boldsymbol{x})) \frac{1}{\rho} \partial_{\boldsymbol{n}}\left(u_{e x t}^{0}+u^{i n c}\right) .
$$

We compute $u^{i n c}+\sum_{\ell=0}^{1} \varepsilon^{\ell} u_{e x t}^{\ell}=\frac{1}{\rho} \varepsilon h \partial_{n}\left(u_{e x t}^{0}+u^{i n c}\right)$ on $\Gamma$. In this case we approach the solution $u_{e x t}^{\varepsilon}$ by the function $v_{[1]}^{\varepsilon}$ that satisfies the Helmholtz equation and the boundary condition $\left(u^{i n c}+v_{[1]}^{\varepsilon}\right)=$ $\varepsilon h \frac{1}{\rho} \partial_{n}\left(u^{i n c}+v_{[1]}^{\varepsilon}\right)$ and we get $u_{e x t}^{\varepsilon}-v_{[1]}^{\varepsilon}=O\left(\varepsilon^{2}\right)$. When $\ell=2$, we obtain the two systems

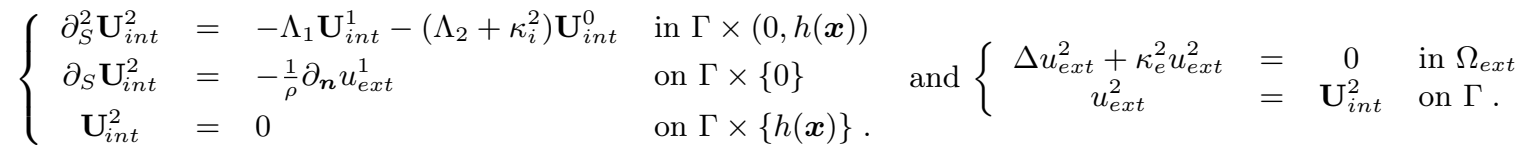

We compute

and we conclude

$$
\partial_{S}^{2} \mathbf{U}_{i n t}^{2}=-\mathcal{H} \frac{1}{\rho} \partial_{n}\left(u_{e x t}^{0}+u^{i n c}\right)
$$

$$
\mathbf{U}_{\text {int }}^{2}(\cdot, S)=-\left(\frac{S^{2}-h^{2}(x)}{2}\right) \mathcal{H} \rho^{-1} \partial_{\boldsymbol{n}}\left(u_{\text {ext }}^{0}+u^{i n c}\right)-(S-h(x)) \rho^{-1} \partial_{\boldsymbol{n}} u_{\text {ext }}^{1} .
$$

We compute $u^{i n c}+\sum_{\ell=0}^{2} \varepsilon^{\ell} u_{e x t}^{\ell}=\frac{1}{2}(\varepsilon h)^{2} \mathcal{H} \frac{1}{\rho} \partial_{\boldsymbol{n}}\left(u_{e x t}^{0}+u^{i n c}\right)+\varepsilon h \frac{1}{\rho} \partial_{\boldsymbol{n}}\left(u^{i n c}+\sum_{\ell=0}^{1} \varepsilon^{\ell} u_{e x t}^{\ell}\right)$ on $\Gamma$. In this case we approach the solution $u_{e x t}^{\varepsilon}$ by the function $v_{[2]}^{\varepsilon}$ that satisfies the Helmholtz equation and the boundary condition $u^{i n c}+v_{[2]}^{\varepsilon}=(\varepsilon h)\left(1+\frac{\varepsilon h}{2} \mathcal{H}\right) \frac{1}{\rho} \partial_{\boldsymbol{n}}\left(u^{i n c}+v_{[2]}^{\varepsilon}\right)$ and we get $u_{e x t}^{\varepsilon}-v_{[2]}^{\varepsilon}=O\left(\varepsilon^{3}\right)$. When $\ell=3$, we obtain the two systems

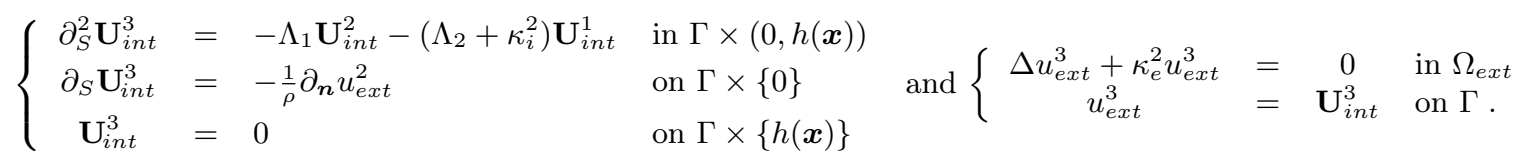

We compute

$$
\partial_{S}^{2} \mathbf{U}_{i n t}^{3}=-S\left(2 \mathcal{H}^{2}-2 \mathcal{G}\right) \frac{1}{\rho} \partial_{\boldsymbol{n}}\left(u_{\text {ext }}^{0}+u^{i n c}\right)-\mathcal{H} \frac{1}{\rho} \partial_{\boldsymbol{n}} u_{\text {ext }}^{1}+\left(\Delta_{\Gamma}+\kappa_{i}^{2}\right)(S-h(\boldsymbol{x})) \frac{1}{\rho} \partial_{\boldsymbol{n}}\left(u_{\text {ext }}^{0}+u^{i n c}\right),
$$

and we conclude

$$
\begin{aligned}
\mathbf{U}_{\text {int }}^{3}(\cdot, S)= & -\left(\frac{S^{3}-h^{3}(x)}{6}\right)\left[\left(2 \mathcal{H}^{2}-2 \mathcal{G}\right) \rho^{-1} \partial_{\boldsymbol{n}}\left(u_{\text {ext }}^{0}+u^{i n c}\right)-\left(\Delta_{\Gamma}+\kappa_{i}^{2}\right) \rho^{-1} \partial_{\boldsymbol{n}}\left(u_{\text {ext }}^{0}+u^{i n c}\right)\right] \\
& -\left(\frac{S^{2}-h^{2}(x)}{2}\right)\left[\mathcal{H} \rho^{-1} \partial_{\boldsymbol{n}} u_{\text {ext } \mid \Gamma}^{1}+\left(\Delta_{\Gamma}+\kappa_{i}^{2}\right) h \rho^{-1} \partial_{\boldsymbol{n}}\left(u_{\text {ext }}^{0}+u^{i n c}\right)\right] \\
& -(S-h(x)) \rho^{-1} \partial_{\boldsymbol{n}} u_{\text {ext }}^{2} .
\end{aligned}
$$

We compute $u^{i n c}+\sum_{\ell=0}^{3} \varepsilon^{\ell} u_{e x t}^{\ell}=(\varepsilon h)^{2}\left[\frac{1}{3}(\varepsilon h)\left(\kappa_{i}^{2}+\mathcal{H}^{2}-\mathcal{G}\right) \mathrm{I}+\frac{1}{2}(\varepsilon h) \Delta_{\Gamma}-\frac{1}{6} \Delta_{\Gamma}(\varepsilon h)\right] \frac{1}{\rho} \partial_{n}\left(u_{e x t}^{0}+u^{i n c}\right)+$ $\frac{1}{2}(\varepsilon h)^{2} \mathcal{H} \frac{1}{\rho} \partial_{\boldsymbol{n}}\left(u^{i n c}+\sum_{\ell=0}^{1} \varepsilon^{\ell} u_{e x t}^{\ell}\right)+\varepsilon h \frac{1}{\rho} \partial_{\boldsymbol{n}}\left(u^{i n c}+\sum_{\ell=0}^{2} \varepsilon^{\ell} u_{\text {ext }}^{\ell}\right)$ on $\Gamma$. In this case we approach the solution $u_{\text {ext }}^{\varepsilon}$ by the function $v_{[3]}^{\varepsilon}$ that satisfies the Helmholtz equation and the boundary condition $u^{i n c}+v_{[3]}^{\varepsilon}=$ $\left[\varepsilon h\left(1+\frac{\varepsilon h}{2} \mathcal{H}+\frac{(\varepsilon h)^{2}}{3}\left(\kappa_{i}^{2}+\mathcal{H}^{2}-\mathcal{G}\right)\right) \mathrm{I}+\frac{(\varepsilon h)^{3}}{2} \Delta_{\Gamma}-\frac{(\varepsilon h)^{2}}{6} \Delta_{\Gamma}(\varepsilon h)\right] \frac{1}{\rho} \partial_{\boldsymbol{n}}\left(u^{i n c}+v_{[3]}^{\varepsilon}\right)$ and we get $u_{\text {ext }}^{\varepsilon}-v_{[3]}^{\varepsilon}=$ $O\left(\varepsilon^{4}\right)$. 
Proposition 3.2. The GIBCs modeling sound-hard obstacles coated by thin layers with a variable thikness are given for $N=0,1,2$ by

$$
\partial_{\boldsymbol{n}}\left(v_{[N]}^{\varepsilon h}+u^{i n c}\right)+B^{\varepsilon h, N}\left(v_{[N]}^{\varepsilon h}+u^{i n c}\right)=0
$$

where

$$
\begin{gathered}
B^{\varepsilon h, 0}=0, \quad B^{\varepsilon h, 1}=\rho\left[\operatorname{div}_{\Gamma}(\varepsilon h) \nabla_{\Gamma}+(\varepsilon h) \kappa_{i}^{2} \mathrm{I}\right], \\
B^{\varepsilon h, 2}=\rho\left[\operatorname{div}_{\Gamma}(\varepsilon h)\left(1+(\varepsilon h)\left(\mathcal{R}-\frac{1}{2} \mathcal{H}\right)\right) \nabla_{\Gamma}+(\varepsilon h)\left(1-\frac{1}{2}(\varepsilon h) \mathcal{H}\right) \kappa_{i}^{2} \mathrm{I}\right], \\
B^{\varepsilon h, 3}=\operatorname{div}_{\Gamma} \varepsilon h\left(1+\frac{\varepsilon h}{2}(2 \mathcal{R}-\mathcal{H})+\frac{(\varepsilon h)^{2}}{3}\left(2 \mathcal{R}^{2}-\mathcal{H} \mathcal{R}\right)\right) \nabla_{\Gamma}+\varepsilon h\left(1-\frac{\varepsilon h}{2} \mathcal{H}+\frac{(\varepsilon h)^{2}}{3} \mathcal{G}\right) \kappa_{i}^{2} \mathrm{I} \\
-\frac{(\varepsilon h)^{3}}{6}\left[\Delta_{\Gamma}+\kappa_{i}^{2} \mathrm{I}\right]^{2}+\frac{(\varepsilon h)^{2}}{2}\left[\Delta_{\Gamma}+\kappa_{i}^{2} \mathrm{I}\right]\left[\operatorname{div}_{\Gamma}(\varepsilon h) \nabla_{\Gamma}+\kappa_{i}^{2}(\varepsilon h) \mathrm{I}\right] \\
+\frac{1}{2} \nabla_{\Gamma}(\varepsilon h) \cdot \nabla_{\Gamma}\left[\operatorname{div}_{\Gamma}(\varepsilon h)^{2} \nabla_{\Gamma}+\kappa_{i}^{2}(\varepsilon h)^{2} \mathrm{I}\right] .
\end{gathered}
$$

Proof. The rank $\ell=0$ allows us to compute $\mathbf{U}_{i n t}^{0}$ only. We obtain the system

$$
\left\{\begin{array}{ccccc}
\partial_{S}^{2} \mathbf{U}_{i n t}^{0} & = & 0 & & \text { in } \Gamma \times(0, h(\boldsymbol{x})) \\
\partial_{S} \mathbf{U}_{i n t}^{0} & = & 0 & & \text { on } \Gamma \times\{h(\boldsymbol{x})\} \\
\mathbf{U}_{i n t}^{0} & = & \left(u^{i n c}+u_{e x t}^{0}\right) & & \text { on } \Gamma \times\{0\}
\end{array}\right.
$$

The first equation implies that $\mathbf{U}_{e x t}^{0}(\boldsymbol{x}, S)$ is a polynomial function of degre 1 in the variable $S$. The second equation implies that the leading coefficient is 0 and the third equation gives the constant term. We conclude that

$$
\mathbf{U}_{i n t}^{0}(\cdot, S)=\left(u^{i n c}+u_{e x t}^{0}\right) .
$$

When $\ell=1$, we obtain the two systems

$$
\left\{\begin{array} { c c c l } 
{ \partial _ { S } ^ { 2 } \mathbf { U } _ { i n t } ^ { 1 } = } & { - \Lambda _ { 1 } \mathbf { U } _ { i n t } ^ { 0 } = 0 } & { \text { in } \Gamma \times ( 0 , h ( \boldsymbol { x } ) ) } \\
{ \partial _ { S } \mathbf { U } _ { i n t } ^ { 1 } = } & { \mathcal { H } \partial _ { S } \mathbf { U } _ { i n t } ^ { 0 } = 0 } & { \text { on } \Gamma \times \{ h ( \boldsymbol { x } ) \} } \\
{ \mathbf { U } _ { i n t } ^ { 1 } } & { = } & { u _ { e x t } ^ { 1 } } & { \text { on } \Gamma \times \{ 0 \} . }
\end{array} \text { and } \left\{\begin{array}{ccc}
\Delta u_{\text {ext }}^{0}+\kappa_{e}^{2} u_{\text {ext }}^{0}= & 0 & \text { in } \Omega_{\text {ext }} \\
\partial_{\boldsymbol{n}}\left(u^{i n c}+u_{e x t}^{0}\right)=-\rho \partial_{S} \mathbf{U}_{i n t}^{1} & \text { on } \Gamma .
\end{array}\right.\right.
$$

We conclude with similar arguments that

$$
\mathbf{U}_{\text {int }}^{1}(\cdot, S)=u_{\text {ext }}^{1} .
$$

We compute $\partial_{\boldsymbol{n}}\left(u^{i n c}+u_{e x t}^{0}\right)=0$ on $\Gamma$. In this case we approach $u_{e x t}^{\varepsilon}$ by the function $v_{[0]}^{\varepsilon}=u_{0}^{e x t}$ and then $\partial_{n}\left(u_{e x t}^{\varepsilon}-v_{[0]}^{\varepsilon}\right)=O(\varepsilon)$. When $\ell=2$, we obtain the two systems

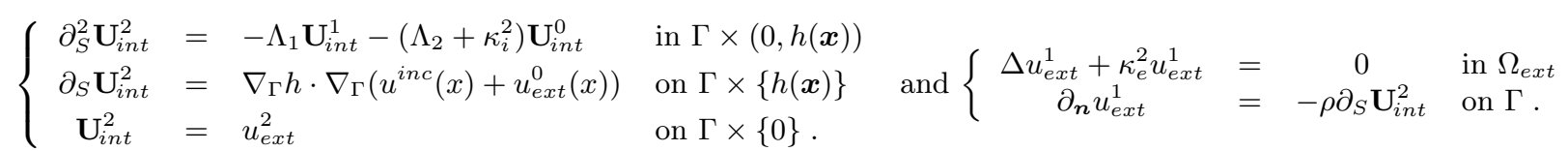

We compute

and we conclude

$$
\partial_{S}^{2} \mathbf{U}_{i n t}^{2}=-\left(\Delta_{\Gamma}+\kappa_{i}^{2}\right)\left(u^{i n c}+u_{e x t}^{0}\right)
$$

$$
\mathbf{U}_{i n t}^{2}(\cdot, S)=-\left(\frac{S^{2}}{2}-S h(x)\right)\left(\Delta_{\Gamma}+\kappa_{i}^{2}\right)\left(u^{i n c}+u_{e x t}^{0}\right)+S \nabla_{\Gamma} h \cdot \nabla_{\Gamma}\left(u^{i n c}+u_{e x t}^{0}\right)+u_{e x t}^{2},
$$

We compute $\partial_{\boldsymbol{n}}\left(u^{i n c}+\sum_{\ell=0}^{1} \varepsilon^{\ell} u_{e x t}^{\ell}\right)=-\rho\left(\operatorname{div}_{\Gamma}(\varepsilon h) \nabla_{\Gamma}+(\varepsilon h) \kappa_{i}^{2}\right)\left(u^{i n c}+u_{e x t}^{0}\right)$ on $\Gamma$. In this case we approach the solution $u_{\text {ext }}^{\varepsilon}$ by the function $v_{[1]}^{\varepsilon}$ that satisfies the Helmholtz equation and the boundary condition $\partial_{\boldsymbol{n}}\left(u^{i n c}+v_{[1]}^{\varepsilon}\right)=-\rho\left(\operatorname{div}_{\Gamma}(\varepsilon h) \nabla_{\Gamma}+(\varepsilon h) \kappa_{i}^{2}\right)\left(u^{i n c}+v_{[1]}^{\varepsilon}\right)$ and we get $\partial_{\boldsymbol{n}}\left(u_{e x t}^{\varepsilon}-v_{[1]}^{\varepsilon}\right)=O\left(\varepsilon^{2}\right)$. When $\ell=3$, we obtain the two systems

$$
\left\{\begin{array}{rlrl}
\partial_{S}^{2} \mathbf{U}_{i n t}^{3}= & -\Lambda_{1} \mathbf{U}_{i n t}^{2}-\left(\Lambda_{2}+\kappa_{i}^{2}\right) \mathbf{U}_{i n t}^{1}-\Lambda_{3} \mathbf{U}_{i n t}^{0} & & \text { in } \Gamma \times(0, h(\boldsymbol{x})) \\
\partial_{S} \mathbf{U}_{i n t}^{3}= & h \mathcal{H} \partial_{S} \mathbf{U}_{i n t}^{2}-h^{2} \mathcal{G} \partial_{S} \mathbf{U}_{i n t}^{1}+\nabla_{\Gamma} h \cdot \nabla_{\Gamma} \mathbf{U}_{i n t}^{1} & \\
& +h \nabla_{\Gamma} h \cdot(2 \mathcal{R}-\mathcal{H}) \nabla_{\Gamma} \mathbf{U}_{i n t}^{0} & & \text { on } \Gamma \times\{h(\boldsymbol{x})\} \\
\mathbf{U}_{i n t}^{3}= & u_{e x t}^{3} & & \text { on } \Gamma \times\{0\}
\end{array}\right.
$$


and

We compute

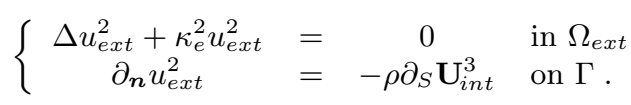

$$
\begin{aligned}
\partial_{S}^{2} \mathbf{U}_{i n t}^{3}(\cdot, S)= & -(S-h(x)) \mathcal{H}\left(\Delta_{\Gamma}+\kappa_{i}^{2}\right)\left(u^{i n c}+u_{\text {ext }}^{0}\right)+\mathcal{H} \nabla_{\Gamma} h \cdot \nabla_{\Gamma}\left(u^{i n c}+u_{\text {ext }}^{0}\right) \\
& -\left(\Delta_{\Gamma}+\kappa_{i}^{2}\right) u_{\text {ext } \mid \Gamma}^{1}-S\left(\operatorname{div}_{\Gamma}(2 \mathcal{R}-\mathcal{H}) \nabla_{\Gamma}+\mathcal{H} \Delta_{\Gamma}\right)\left(u^{i n c}+u_{\text {ext }}^{0}\right)
\end{aligned}
$$

and

$$
\partial_{S} \mathbf{U}_{i n t}^{3}(\cdot, h(x))=h \mathcal{H} \nabla_{\Gamma} h \cdot \nabla_{\Gamma}\left(u^{i n c}+u_{\text {ext }}^{0}\right)+\nabla_{\Gamma} h \cdot \nabla_{\Gamma} u_{\text {ext } \mid \Gamma}^{1}+h \nabla_{\Gamma} h \cdot(2 \mathcal{R}-\mathcal{H}) \nabla_{\Gamma}\left(u^{i n c}+u_{\text {ext }}^{0}\right) .
$$

We conclude

$$
\begin{aligned}
\mathbf{U}_{i n t}^{3}(\cdot, S)= & -\left(\frac{S^{3}}{6}-\frac{S h^{2}(x)}{2}\right)\left[\mathcal{H}\left(\Delta_{\Gamma}+\kappa_{i}^{2}\right)\left(u^{i n c}+u_{e x t}^{0}\right)+\left(\operatorname{div}_{\Gamma}(2 \mathcal{R}-\mathcal{H}) \nabla_{\Gamma}+\mathcal{H} \Delta_{\Gamma}\right)\left(u^{i n c}+u_{\text {ext }}^{0}\right)\right] \\
& +\left(\frac{S^{2}}{2}-S h(x)\right)\left[h \mathcal{H}\left(\Delta_{\Gamma}+\kappa_{i}^{2}\right)\left(u^{i n c}+u_{e x t}^{0}\right)+\mathcal{H} \nabla_{\Gamma} h \cdot \nabla_{\Gamma}\left(u^{i n c}+u_{\text {ext }}^{0}\right)-\left(\Delta_{\Gamma}+\kappa_{i}^{2}\right) u_{\text {ext } \mid \Gamma}^{1}\right] \\
& +S\left[\nabla_{\Gamma} h \cdot \nabla_{\Gamma} u_{\text {ext } \mid \Gamma}^{1}+h \nabla_{\Gamma} h \cdot 2 \mathcal{R} \nabla_{\Gamma}\left(u^{i n c}+u_{\text {ext }}^{0}\right)\right]+u_{\text {ext }}^{3} .
\end{aligned}
$$

We compute $\partial_{\boldsymbol{n}}\left(u^{i n c}+\sum_{\ell=0}^{2} \varepsilon^{\ell} u_{\text {ext }}^{\ell}\right)=-\frac{1}{2} \rho\left[\operatorname{div}_{\Gamma}(\varepsilon h)^{2}(2 \mathcal{R}-\mathcal{H} \mathrm{I}) \nabla_{\Gamma}-(\varepsilon h)^{2} \mathcal{H} \kappa_{i}^{2} \mathrm{I}\right]\left(u_{\text {ext }}^{0}+u^{i n c}\right)$

$-\rho\left(\operatorname{div}_{\Gamma}(\varepsilon h) \nabla_{\Gamma}+(\varepsilon h) \kappa_{i}^{2}\right)\left(u^{i n c}+\sum_{\ell=0}^{1} \varepsilon^{\ell} u_{e x t}^{\ell}\right)$. In this case we approach the solution $u_{e x t}^{\varepsilon}$ by the function $v_{[2]}^{\varepsilon}$ that satisfies the Helmholtz equation and the boundary condition

$\partial_{\boldsymbol{n}}\left(u^{i n c}+v_{[2]}^{\varepsilon}\right)=-\rho\left(\varepsilon\left[\Delta_{\Gamma}+\kappa_{i}^{2} \mathrm{I}\right]-\frac{1}{2} \varepsilon^{2}\left[\operatorname{div}_{\Gamma}(2 \mathcal{R}-\mathcal{H} \mathrm{I}) \nabla_{\Gamma}-\mathcal{H} \kappa_{i}^{2} \mathrm{I}\right]\right)\left(u^{i n c}+v_{[2]}^{\varepsilon}\right)$ and we get $\partial_{\boldsymbol{n}}\left(u_{\text {ext }}^{\varepsilon}-\right.$ $\left.v_{[2]}^{\varepsilon}\right)=O\left(\varepsilon^{3}\right)$.

When $\ell=4$, we obtain the two systems

$$
\left\{\begin{array}{rlrl}
\partial_{S}^{2} \mathbf{U}_{i n t}^{4}= & -\Lambda_{1} \mathbf{U}_{i n t}^{3}-\left(\Lambda_{2}+\kappa_{i}^{2}\right) \mathbf{U}_{i n t}^{2}-\Lambda_{3} \mathbf{U}_{i n t}^{1}-\Lambda_{4} \mathbf{U}_{i n t}^{0} & \text { in } \Gamma \times(0, h) \\
\partial_{S} \mathbf{U}_{i n t}^{4}= & h \mathcal{H} \partial_{S} \mathbf{U}_{i n t}^{3}-h^{2} \mathcal{G} \partial_{S} \mathbf{U}_{i n t}^{2}+\nabla_{\Gamma} h \cdot \nabla_{\Gamma} \mathbf{U}_{i n t}^{2} & \\
& +h \nabla_{\Gamma} h \cdot(2 \mathcal{R}-\mathcal{H}) \nabla_{\Gamma} \mathbf{U}_{i n t}^{1}+h^{2} \nabla_{\Gamma} h \cdot\left(2 \mathcal{R}^{2}-\mathcal{H R}\right) \nabla_{\Gamma} \mathbf{U}_{i n t}^{0} & & \text { on } \Gamma \times\{h\} \\
\mathbf{U}_{i n t}^{4}= & u_{e x t}^{4} & \text { on } \Gamma \times\{0\} .
\end{array}\right.
$$

and

We obtain

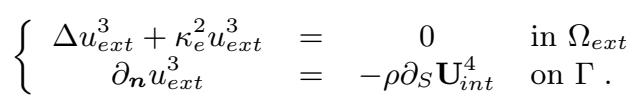

$$
\begin{aligned}
& \partial_{S} \mathbf{U}_{i n t}^{4}(\cdot, S) \\
& =-\mathcal{H}\left(\frac{1}{6}\left(S^{3}-h^{3}\right)-\frac{1}{2}(S-h) h^{2}\right)\left[\mathcal{H}\left(\Delta_{\Gamma}+\kappa_{i}^{2}\right)\left(u^{i n c}+u_{\text {ext }}^{0}\right)+\left(\operatorname{div}_{\Gamma}(2 \mathcal{R}-\mathcal{H}) \nabla_{\Gamma}+\mathcal{H} \Delta_{\Gamma}\right)\left(u^{i n c}+u_{\text {ext }}^{0}\right)\right] \\
& +\mathcal{H} \frac{1}{2}(S-h)^{2}\left[h \mathcal{H}\left(\Delta_{\Gamma}+\kappa_{i}^{2}\right)\left(u^{i n c}+u_{e x t}^{0}\right)+\mathcal{H} \nabla_{\Gamma} h \cdot \nabla_{\Gamma}\left(u^{i n c}+u_{e x t}^{0}\right)-\left(\Delta_{\Gamma}+\kappa_{i}^{2}\right) u_{e x t \mid \Gamma}^{1}\right] \\
& +(S-h) \mathcal{H}\left[\nabla_{\Gamma} h \cdot \nabla_{\Gamma} u_{e x t \mid \Gamma}^{1}+h \nabla_{\Gamma} h \cdot 2 \mathcal{R} \nabla_{\Gamma}\left(u^{i n c}+u_{e x t}^{0}\right)\right]-(S-h)\left(\Delta_{\Gamma}+\kappa_{i}^{2}\right) u_{e x t}^{2} \\
& +\frac{\left(S^{3}-h^{3}\right)}{6}\left(\Delta_{\Gamma}+\kappa_{i}^{2}\right)\left(\Delta_{\Gamma}+\kappa_{i}^{2}\right)\left(u^{i n c}+u_{e x t}^{0}\right)-\frac{1}{2}\left(S^{2}-h^{2}\right)\left(\operatorname{div}_{\Gamma}(2 \mathcal{R}-\mathcal{H}) \nabla_{\Gamma}+\mathcal{H} \Delta_{\Gamma}\right) u_{e x t \mid \Gamma}^{1} \\
& -\frac{\left(S^{2}-h^{2}\right)}{2}\left(\Delta_{\Gamma}+\kappa_{i}^{2}\right)\left[h\left(\Delta_{\Gamma}+\kappa_{i}^{2}\right)\left(u^{i n c}+u_{e x t}^{0}\right)+\nabla_{\Gamma} h \cdot \nabla_{\Gamma}\left(u^{i n c}+u_{e x t}^{0}\right)\right] \\
& -\left(\mathcal{H}^{2}-2 \mathcal{G}\right)\left[\left(\frac{1}{3}\left(S^{3}-h^{3}\right)-\frac{1}{2}\left(S^{2}-h^{2}\right) h\right)\left(\Delta_{\Gamma}+\kappa_{i}^{2}\right)\left(u^{i n c}+u_{e x t}^{0}\right)-\frac{1}{2}\left(S^{2}-h^{2}\right) \nabla_{\Gamma} h \cdot \nabla_{\Gamma}\left(u^{i n c}+u_{\text {ext }}^{0}\right)\right] \\
& -\frac{1}{3}\left(S^{3}-h^{3}\right)\left(\operatorname{div}_{\Gamma}\left(2 \mathcal{R}^{2}-\mathcal{H} \mathcal{R}\right) \nabla_{\Gamma}+\mathcal{H} \operatorname{div}_{\Gamma}(2 \mathcal{R}-\mathcal{H}) \nabla_{\Gamma}+\left(\mathcal{H}^{2}-\mathcal{G}\right) \Delta_{\Gamma}\right)\left(u^{i n c}+u_{\text {ext }}^{0}\right) \\
& +h \mathcal{H}\left[\nabla_{\Gamma} h \cdot \nabla_{\Gamma} u_{e x t \mid \Gamma}^{1}+h \nabla_{\Gamma} h \cdot 2 \mathcal{R} \nabla_{\Gamma}\left(u^{i n c}+u_{\text {ext }}^{0}\right)\right]-h^{2} \mathcal{G} \nabla_{\Gamma} h \cdot \nabla_{\Gamma}\left(u^{i n c}+u_{\text {ext }}^{0}\right) \\
& +\nabla_{\Gamma} h \cdot \nabla_{\Gamma}\left[\frac{1}{2} h^{2}\left(\Delta_{\Gamma}+\kappa_{i}^{2}\right)\left(u^{i n c}+u_{e x t}^{0}\right)+h \nabla_{\Gamma} h \cdot \nabla_{\Gamma}\left(u^{i n c}+u_{e x t}^{0}\right)+u_{e x t}^{2}\right] \\
& +h \nabla_{\Gamma} h \cdot(2 \mathcal{R}-\mathcal{H}) \nabla_{\Gamma} u_{\text {ext }}^{1}+h^{2} \nabla_{\Gamma} h \cdot\left(2 \mathcal{R}^{2}-\mathcal{H} \mathcal{R}\right) \nabla_{\Gamma}\left(u^{i n c}+u_{\text {ext }}^{0}\right) .
\end{aligned}
$$


We compute $\partial_{n} u_{e x t}^{3}=-\rho \partial_{S} \mathbf{U}_{i n t}^{4}(\cdot, 0)$ and obtain on $\Gamma$

$$
\begin{aligned}
\partial_{\boldsymbol{n}} u_{\text {ext }}^{3}= & -\rho\left(\operatorname{div}_{\Gamma} h \nabla_{\Gamma}+h \kappa_{i}^{2}\right) u_{\text {ext }}^{2}-\rho\left[\operatorname{div}_{\Gamma} h^{2}\left(\mathcal{R}-\frac{1}{2} \mathcal{H}\right) \nabla_{\Gamma}-\frac{1}{2} h^{2} \mathcal{H} \kappa_{i}^{2}\right] u_{\text {ext }}^{1} \\
& -\frac{\rho}{3} \operatorname{div}_{\Gamma} h^{3}\left(2 \mathcal{R}^{2}-\mathcal{H} \mathcal{R}\right) \nabla_{\Gamma}\left(u^{i n c}+u_{\text {ext }}^{0}\right)-\frac{\rho}{3} h^{3} \mathcal{G} \kappa_{i}^{2}\left(u^{i n c}+u_{\text {ext }}^{0}\right) \\
& +\frac{\rho h^{3}}{6}\left[\Delta_{\Gamma}+\kappa_{i}^{2}\right]^{2}\left(u^{i n c}+u_{\text {ext }}^{0}\right)-\frac{\rho h^{2}}{2}\left[\Delta_{\Gamma}+\kappa_{i}^{2}\right]\left[\operatorname{div}_{\Gamma} h \nabla_{\Gamma}+\kappa_{i}^{2} h\right]\left(u^{i n c}+u_{\text {ext }}^{0}\right) \\
& -\frac{\rho}{2} \nabla_{\Gamma} h \cdot \nabla_{\Gamma}\left[\operatorname{div}_{\Gamma} h^{2} \nabla_{\Gamma}+\kappa_{i}^{2} h^{2}\right]\left(u^{i n c}+u_{e x t}^{0}\right) .
\end{aligned}
$$

We construct the approximate solution $v_{[3]}^{\varepsilon}$ in the same way as previously and we get $\partial_{\boldsymbol{n}}\left(u_{e x t}^{\varepsilon}-v_{[3]}^{\varepsilon}\right)=$ $O\left(\varepsilon^{4}\right)$.

\section{Construction of GIBCs for the shape derivatives}

In the remaining of the paper, we assume that the layer $\Omega_{i n t}^{\varepsilon}$ has a constant thickness $\varepsilon>0$ which means $\forall \boldsymbol{x} \in \Gamma, h(\boldsymbol{x})=1$. This section is devoted to the shape derivative analysis of the solution to the thin layer transmission problem. In paragraph 4.1 we give a characterisation of the shape derivative as a solution to a new transmission problem with non vanishing jumps. In paragraph 4.2 we construct the GIBC statisfied by the approximate shape derivative $w_{[N]}^{\varepsilon}$ for $N=0,1,2$.

\subsection{Characterization of the shape derivative}

From now on, we choose a fixed reference domain $\Omega$ with a closed and orientable boundary $\Gamma$ of class $\mathscr{C}^{k}$, with $k$ as great as we need, and we consider variations generated by transformations of the form $\boldsymbol{x} \mapsto \boldsymbol{x}+\boldsymbol{\theta}(\boldsymbol{x})$ of point $\boldsymbol{x}$ in the space $\mathbb{R}^{3}$, where $\boldsymbol{\theta}$ is a smooth vector function defined in a neighborhood of $\Gamma$. The functions $\boldsymbol{\theta}$ are assumed to be sufficiently small elements in an open subset $\mathcal{O}$ of the Banach space $\mathscr{C}^{k}\left(\Gamma, \mathbb{R}^{3}\right)$ in order that $(\mathrm{I}+\boldsymbol{\theta})$ is a diffeomorphism from $\Gamma$ to $\Gamma_{\boldsymbol{\theta}}:=\left\{\boldsymbol{x}_{\boldsymbol{\theta}}=\boldsymbol{x}+\boldsymbol{\theta}(\boldsymbol{x}) ; \boldsymbol{x} \in \Gamma\right\}$. By $\boldsymbol{n}_{\boldsymbol{\theta}}$ we denote the outward unit normal vector to $\Gamma_{\boldsymbol{\theta}}$ and we set

$$
\Gamma_{\boldsymbol{\theta}}^{\varepsilon}:=\left\{\boldsymbol{y}_{\boldsymbol{\theta}}=\boldsymbol{x}_{\boldsymbol{\theta}}-\varepsilon \boldsymbol{n}_{\boldsymbol{\theta}}\left(\boldsymbol{x}_{\boldsymbol{\theta}}\right) \mid \boldsymbol{x}_{\boldsymbol{\theta}} \in \Gamma_{\boldsymbol{\theta}}\right\} .
$$

The transformation $\tau_{\varepsilon}^{-1}$ maps the restriction $\boldsymbol{\theta}_{\mid \Gamma}$ of $\boldsymbol{\theta}$ to $\Gamma$ to the function defined on $\Gamma^{\varepsilon}$ by $\left(\tau_{\varepsilon}^{-1} \boldsymbol{\theta}_{\mid \Gamma}\right)(\boldsymbol{x}+$ $\varepsilon \boldsymbol{n}(\boldsymbol{x}))=\boldsymbol{\theta}_{\mid \Gamma}(\boldsymbol{x})$. We have $\Gamma_{\boldsymbol{\theta}}=(\mathrm{I}+\boldsymbol{\theta}) \Gamma$ but in general $\Gamma_{\boldsymbol{\theta}}^{\varepsilon} \neq\left(\mathrm{I}+\tau_{\varepsilon}^{-1} \boldsymbol{\theta}\right) \Gamma^{\varepsilon}$. Indeed, let $\boldsymbol{y}_{\boldsymbol{\theta}} \in \Gamma_{\boldsymbol{\theta}}^{\varepsilon}$ and $\boldsymbol{y} \in \Gamma^{\varepsilon}$, then

$$
\begin{aligned}
\boldsymbol{y}_{\boldsymbol{\theta}}-\boldsymbol{y}=\boldsymbol{x}_{\boldsymbol{\theta}}-\varepsilon \boldsymbol{n}_{\boldsymbol{\theta}}\left(\boldsymbol{x}_{\boldsymbol{\theta}}\right)-(\boldsymbol{x}-\varepsilon \boldsymbol{n}(\boldsymbol{x})) & =\boldsymbol{\theta}(\boldsymbol{x})-\varepsilon\left(\boldsymbol{n}_{\boldsymbol{\theta}}(\boldsymbol{x}+\boldsymbol{\theta}(\boldsymbol{x}))-\boldsymbol{n}(\boldsymbol{x})\right) \\
& =\boldsymbol{\theta}(\boldsymbol{x})+\varepsilon\left[\nabla_{\Gamma} \boldsymbol{\theta}(\boldsymbol{x})\right] \boldsymbol{n}(\boldsymbol{x})+O\left(\|\boldsymbol{\theta}\|_{\mathscr{C}}^{2}\right) .
\end{aligned}
$$

The following theorem is a direct consequence of the Theorem 4.2 in [24] (see also [1, 20, 21]).

Theorem 4.1. Assume that $\left(u_{i n t}^{\varepsilon, \boldsymbol{\theta}}, u_{\text {ext }}^{\varepsilon, \boldsymbol{\theta}}\right)$ is the solution to the transmission problem (1.1) where $f_{\text {ext }}$ and $g_{\text {ext }}$ are the boundary data of $u^{i n c}(\boldsymbol{x})=e^{i \kappa_{e} \boldsymbol{d} \cdot \boldsymbol{x}}$ on $\Gamma_{\boldsymbol{\theta}}$ and $f_{\text {int }}=0=g_{\text {int }}$ on $\Gamma_{\boldsymbol{\theta}}^{\varepsilon}$. Then the mappings $\boldsymbol{\theta} \mapsto u_{\text {int }}^{\varepsilon, \boldsymbol{\theta}}$ and $\boldsymbol{\theta} \mapsto u_{\text {ext }}^{\varepsilon, \boldsymbol{\theta}}$ are Fréchet differentiable at $\boldsymbol{\theta}=0$ and the derivative $\left(\dot{u}_{\text {int }}^{\varepsilon}, \dot{u}_{\text {ext }}^{\varepsilon}\right)$ in the direction $\boldsymbol{\theta} \in \mathscr{C}^{k}\left(\Gamma, \mathbb{R}^{3}\right)$ is the radiating solution to the transmission problem

$$
\left\{\begin{array}{clll}
\Delta \dot{u}_{i n t}^{\varepsilon}+\kappa_{i}^{2} \dot{u}_{\text {int }}^{\varepsilon} & =0 & & \text { in } \Omega_{\text {int }}^{\varepsilon} \\
\Delta \dot{u}_{\text {ext }}^{\varepsilon}+\kappa_{e}^{2} \dot{u}_{\text {ext }}^{\varepsilon} & =0 & & \text { in } \Omega_{\text {ext }} \\
\rho \partial_{\boldsymbol{n}} \dot{u}_{\text {int }}^{\varepsilon}-\partial_{\boldsymbol{n}} \dot{u}_{\text {ext }}^{\varepsilon} & =\left[(\rho-1) \operatorname{div}_{\Gamma}(\boldsymbol{\theta} \cdot \boldsymbol{n}) \nabla_{\Gamma}+(\boldsymbol{\theta} \cdot \boldsymbol{n})\left(\rho \kappa_{i}^{2}-\kappa_{e}^{2}\right)\right]\left(u^{i n c}+u_{\text {ext }}^{\varepsilon}\right) & & \text { on } \Gamma \\
\dot{u}_{i n t}^{\varepsilon}-\dot{u}_{\text {ext }}^{\varepsilon} & =-(\boldsymbol{\theta} \cdot \boldsymbol{n})\left(\frac{1}{\rho}-1\right) \partial_{\boldsymbol{n}}\left(u^{i n c}+u_{\text {ext }}^{\varepsilon}\right) & & \text { on } \Gamma,
\end{array}\right.
$$

with either a non vanishing Dirichlet boundary condition on $\Gamma^{\varepsilon}$

$$
\dot{u}_{i n t}^{\varepsilon}=-\left(\tau_{\varepsilon}^{-1}(\boldsymbol{\theta} \cdot \boldsymbol{n})\right) \partial_{\boldsymbol{n}^{\varepsilon}} u_{i n t}^{\varepsilon},
$$

or a non vanishing Neumann boundary condition on $\Gamma^{\varepsilon}$

$$
\partial_{\boldsymbol{n}^{\varepsilon}} \dot{u}_{i n t}^{\varepsilon}=\left[\operatorname{div}_{\Gamma^{\varepsilon}}\left(\tau_{\varepsilon}^{-1}(\boldsymbol{\theta} \cdot \boldsymbol{n})\right) \nabla_{\Gamma^{\varepsilon}}+\left(\tau_{\varepsilon}^{-1}(\boldsymbol{\theta} \cdot \boldsymbol{n})\right) \kappa_{i}^{2} \mathrm{I}\right] u_{i n t}^{\varepsilon} .
$$

Proof. Among all the already existing techniques to prove the Fréchet differentiability of the solution we can consider the boundary integral equation approach. Using the results detailed in the Appendix and the material derivative analysis of boundary integral operators presented in [14, 31, 30, we deduce the Fréchet differentiability of the solution. It remains to compute the boundary condition satisfied by the solution. The boundary conditions fullfilled by the derivative on $\Gamma$ are given in Theorem 4.2 in [24]. It remains to compute the boundary data of the derivative on $\Gamma^{\varepsilon}$. 
- In the case of a Dirichlet boundary condition on $\Gamma^{\varepsilon}$, we have

$$
u_{\text {int }}^{\varepsilon, \boldsymbol{\theta}}\left(\boldsymbol{x}+\boldsymbol{\theta}(\boldsymbol{x})-\varepsilon \boldsymbol{n}_{\boldsymbol{\theta}}(\boldsymbol{x}+\boldsymbol{\theta}(\boldsymbol{x}))=0, \quad \text { for all } \boldsymbol{x} \in \Gamma \text { and for all } \boldsymbol{\theta} \in \mathcal{O} .\right.
$$

The material derivative of the normal vector is given in [14, Lemma 4.3$]$ by $\partial_{\boldsymbol{\xi}}\left\{\boldsymbol{n}_{\boldsymbol{\xi}} \circ(\mathrm{I}+\boldsymbol{\xi})\right\}_{\mid \boldsymbol{\xi}=0} \boldsymbol{\theta}=-\left[\nabla_{\Gamma} \boldsymbol{\theta}\right] \boldsymbol{n}$. By differentiation with respect to the boundary parametrization, we have

$$
\begin{aligned}
0= & \dot{u}_{i n t}^{\varepsilon}(\boldsymbol{x}+\varepsilon \boldsymbol{n}(\boldsymbol{x}))+\left(\boldsymbol{\theta}(\boldsymbol{x})+\varepsilon\left[\tau_{\varepsilon} \nabla_{\Gamma^{\varepsilon}} \tau_{\varepsilon}^{-1} \boldsymbol{\theta}(\boldsymbol{x})\right] \boldsymbol{n}(\boldsymbol{x})\right) \cdot\left(\nabla u_{i n t}^{\varepsilon}\right)_{\Gamma^{\varepsilon}}(\boldsymbol{x}+\varepsilon \boldsymbol{n}(\boldsymbol{x})) \\
= & \dot{u}_{i n t}^{\varepsilon}(\boldsymbol{x}+\varepsilon \boldsymbol{n}(\boldsymbol{x}))+(\boldsymbol{\theta}(\boldsymbol{x}) \cdot \boldsymbol{n}(\boldsymbol{x})) \partial_{\boldsymbol{n}^{\varepsilon}} u_{i n t}^{\varepsilon}(\boldsymbol{x}+\varepsilon \boldsymbol{n}(\boldsymbol{x})) \\
& +\left(\boldsymbol{\theta}(\boldsymbol{x})+\varepsilon\left[\tau_{\varepsilon} \nabla_{\Gamma^{\varepsilon}} \tau_{\varepsilon}^{-1} \boldsymbol{\theta}(\boldsymbol{x})\right] \boldsymbol{n}(\boldsymbol{x})\right) \cdot\left(\nabla_{\Gamma^{\varepsilon}} u_{i n t}^{\varepsilon}\right)(\boldsymbol{x}+\varepsilon \boldsymbol{n}(\boldsymbol{x})) \\
= & \dot{u}_{i n t}^{\varepsilon}(\boldsymbol{x}+\varepsilon \boldsymbol{n}(\boldsymbol{x}))+(\boldsymbol{\theta}(\boldsymbol{x}) \cdot \boldsymbol{n}(\boldsymbol{x})) \partial_{\boldsymbol{n}^{\varepsilon}} u_{i n t}^{\varepsilon}(\boldsymbol{x}+\varepsilon \boldsymbol{n}(\boldsymbol{x}))
\end{aligned}
$$

since $u_{i n t}^{\varepsilon}(\boldsymbol{x}+\varepsilon \boldsymbol{n}(\boldsymbol{x}))=0$ for all $\boldsymbol{x} \in \Gamma$.

- In the case of a Neumann boundary condition on $\Gamma^{\varepsilon}$, we have

$$
\partial_{\boldsymbol{n}_{\boldsymbol{\theta}}^{\varepsilon}} u_{i n t}^{\varepsilon, \boldsymbol{\theta}}\left(\boldsymbol{x}+\boldsymbol{\theta}(\boldsymbol{x})-\varepsilon \boldsymbol{n}_{\boldsymbol{\theta}}(\boldsymbol{x}+\boldsymbol{\theta}(\boldsymbol{x}))=0, \text { for all } \boldsymbol{x} \in \Gamma \text { and for all } \boldsymbol{\theta} \in \mathcal{O} .\right.
$$

By differentiation with respect to the boundary parametrization, we have

$$
\begin{aligned}
0= & \partial_{\boldsymbol{n}^{\varepsilon}} \dot{u}_{i n t}^{\varepsilon}(\boldsymbol{x}+\varepsilon \boldsymbol{n}(\boldsymbol{x}))-\left[\tau_{\varepsilon} \nabla_{\Gamma^{\varepsilon}} \tau_{\varepsilon}^{-1}\left(\boldsymbol{\theta}(\boldsymbol{x})+\varepsilon\left[\tau_{\varepsilon} \nabla_{\Gamma^{\varepsilon}} \tau_{\varepsilon}^{-1} \boldsymbol{\theta}(\boldsymbol{x})\right] \boldsymbol{n}(\boldsymbol{x})\right)\right] \boldsymbol{n}(\boldsymbol{x}) \cdot\left(\left.\nabla u_{i n t}^{\varepsilon}\right|_{\Gamma^{\varepsilon}}(\boldsymbol{x}+\varepsilon \boldsymbol{n}(\boldsymbol{x}))\right. \\
& +\boldsymbol{n}(\boldsymbol{x}) \cdot\left[\left(\boldsymbol{\theta}(\boldsymbol{x})+\varepsilon\left[\tau_{\varepsilon} \nabla_{\Gamma^{\varepsilon}} \tau_{\varepsilon}^{-1} \boldsymbol{\theta}(\boldsymbol{x})\right] \boldsymbol{n}(\boldsymbol{x})\right) \cdot \nabla\left(\nabla u_{i n t}^{\varepsilon}\right)_{\Gamma^{\varepsilon}}(\boldsymbol{x}+\varepsilon \boldsymbol{n}(\boldsymbol{x}))\right] \\
= & \partial_{\boldsymbol{n}^{\varepsilon}} \dot{u}_{i n t}^{\varepsilon}(\boldsymbol{x}+\varepsilon \boldsymbol{n}(\boldsymbol{x}))-\left[\tau_{\varepsilon} \nabla_{\Gamma^{\varepsilon}} \tau_{\varepsilon}^{-1}\left(\boldsymbol{\theta}(\boldsymbol{x})+\varepsilon\left[\tau_{\varepsilon} \nabla_{\Gamma^{\varepsilon}} \tau_{\varepsilon}^{-1} \boldsymbol{\theta}(\boldsymbol{x})\right] \boldsymbol{n}(\boldsymbol{x})\right)\right] \boldsymbol{n}(\boldsymbol{x}) \cdot\left(\nabla_{\Gamma^{\varepsilon}} u_{i n t}^{\varepsilon}\right)_{\Gamma^{\varepsilon}}(\boldsymbol{x}+\varepsilon \boldsymbol{n}(\boldsymbol{x})) \\
& +(\boldsymbol{\theta}(\boldsymbol{x}) \cdot \boldsymbol{n}(\boldsymbol{x}))\left(\left.\boldsymbol{n}(\boldsymbol{x}) \cdot \partial_{\boldsymbol{n}^{\varepsilon}} \nabla u_{i n t}^{\varepsilon}\right|_{\Gamma^{\varepsilon}}(\boldsymbol{x}+\varepsilon \boldsymbol{n}(\boldsymbol{x}))\right. \\
& +\boldsymbol{n}(\boldsymbol{x}) \cdot\left[\left(\boldsymbol{\theta}(\boldsymbol{x})+\varepsilon\left[\tau_{\varepsilon} \nabla_{\Gamma^{\varepsilon}} \tau_{\varepsilon}^{-1} \boldsymbol{\theta}(\boldsymbol{x})\right] \boldsymbol{n}(\boldsymbol{x})\right) \cdot \nabla_{\Gamma^{\varepsilon}}\left(\left.\nabla u_{i n t}^{\varepsilon}\right|_{\Gamma^{\varepsilon}}(\boldsymbol{x}+\varepsilon \boldsymbol{n}(\boldsymbol{x}))\right]\right. \\
= & \partial_{\boldsymbol{n}^{\varepsilon}} u_{i n t}^{\varepsilon}(\boldsymbol{x}+\varepsilon \boldsymbol{n}(\boldsymbol{x}))-\left[\tau_{\varepsilon} \nabla_{\Gamma^{\varepsilon}} \tau_{\varepsilon}^{-1}\left(\boldsymbol{\theta}(\boldsymbol{x})+\varepsilon\left[\tau_{\varepsilon} \nabla_{\Gamma^{\varepsilon}} \tau_{\varepsilon}^{-1} \boldsymbol{\theta}(\boldsymbol{x})\right] \boldsymbol{n}(\boldsymbol{x})\right)\right] \boldsymbol{n}(\boldsymbol{x}) \cdot\left(\nabla_{\Gamma^{\varepsilon}} u_{i n t}^{\varepsilon}\right)_{\Gamma^{\varepsilon}}(\boldsymbol{x}+\varepsilon \boldsymbol{n}(\boldsymbol{x})) \\
& +\left.(\boldsymbol{\theta}(\boldsymbol{x}) \cdot \boldsymbol{n}(\boldsymbol{x}))\left(-\kappa_{i}^{2} u_{i n t}^{\varepsilon}-\Delta_{\Gamma^{\varepsilon}} u_{i n t}^{\varepsilon}\right)\right|_{\Gamma^{\varepsilon}}(\boldsymbol{x}+\varepsilon \boldsymbol{n}(\boldsymbol{x})) \\
& -\left(\left.\nabla_{\Gamma^{\varepsilon}}^{\varepsilon} u_{i n t}^{\varepsilon}\right|_{\Gamma^{\varepsilon}}(\boldsymbol{x}+\varepsilon \boldsymbol{n}(\boldsymbol{x})) \cdot\left[\left(\boldsymbol{\theta}(\boldsymbol{x})+\varepsilon\left[\tau_{\varepsilon} \nabla_{\Gamma^{\varepsilon}} \tau_{\varepsilon}^{-1} \boldsymbol{\theta}(\boldsymbol{x})\right] \boldsymbol{n}(\boldsymbol{x})\right) \cdot \nabla_{\Gamma^{\varepsilon}} \boldsymbol{n}(\boldsymbol{x})\right]\right. \\
= & \partial_{\boldsymbol{n}^{\varepsilon}} \dot{u}_{i n t}^{\varepsilon}(\boldsymbol{x}+\varepsilon \boldsymbol{n}(\boldsymbol{x}))-\left[\tau_{\varepsilon} \nabla_{\Gamma^{\varepsilon}} \tau_{\varepsilon}^{-1}(\boldsymbol{\theta}(\boldsymbol{x}) \cdot \boldsymbol{n}(\boldsymbol{x}))\right] \cdot\left(\nabla_{\Gamma^{\varepsilon}} u_{i n t}^{\varepsilon}\right)_{\Gamma^{\varepsilon}}(\boldsymbol{x}+\varepsilon \boldsymbol{n}(\boldsymbol{x})) \\
& +(\boldsymbol{\theta}(\boldsymbol{x}) \cdot \boldsymbol{n}(\boldsymbol{x}))\left(-\kappa_{i}^{2} u_{i n t}^{\varepsilon}-\left.\Delta_{\Gamma^{\varepsilon}} u_{i n t}^{\varepsilon}\right|_{\Gamma^{\varepsilon}}(\boldsymbol{x}+\varepsilon \boldsymbol{n}(\boldsymbol{x}))\right. \\
= & \partial_{\boldsymbol{n}^{\varepsilon}} \dot{u}_{i n t}^{\varepsilon}(\boldsymbol{x}+\varepsilon \boldsymbol{n}(\boldsymbol{x}))-\kappa_{i}^{2}(\boldsymbol{\theta}(\boldsymbol{x}) \cdot \boldsymbol{n}(\boldsymbol{x})) u_{i n t}^{\varepsilon}(\boldsymbol{x}+\varepsilon \boldsymbol{n}(\boldsymbol{x})) \\
& -\operatorname{div}_{\Gamma^{\varepsilon}} \tau_{\varepsilon}^{-1}(\boldsymbol{\theta} \cdot \boldsymbol{n}) \tau_{\varepsilon}\left(\left.\nabla_{\Gamma^{\varepsilon}} u_{i n t}^{\varepsilon}\right|_{\Gamma^{\varepsilon}}(\boldsymbol{x}+\varepsilon \boldsymbol{n}(\boldsymbol{x})) .\right.
\end{aligned}
$$

Now, we assume $\dot{u}_{i n t}^{\varepsilon}(\boldsymbol{y})=\dot{\mathbf{U}}_{i n t}^{\varepsilon}(\boldsymbol{x}, S)=\sum_{\ell \geq 0} \varepsilon^{\ell} \mathbf{U}_{i n t}^{\ell}(\boldsymbol{x}, S)$ in $\Gamma \times[0,1]$ and $\dot{u}_{\text {ext }}^{\varepsilon}(\boldsymbol{y})=\sum_{\ell \geq 0} \varepsilon^{\ell} u_{\text {ext }}^{\ell}(\boldsymbol{y})$ in $\Omega_{\text {ext }}$. If we use the asymptotic expansions of the gradient 2.5 and the divergence 2.6 we obtain

$$
\left(\tau_{\varepsilon} \operatorname{div}_{\Gamma^{\varepsilon}} \tau_{\varepsilon}^{-1}\right)(\boldsymbol{\theta} \cdot \boldsymbol{n})\left(\tau_{\varepsilon} \nabla_{\Gamma^{\varepsilon}} \tau_{\varepsilon}^{-1}\right)=\operatorname{div}_{\Gamma}(\boldsymbol{\theta} \cdot \boldsymbol{n}) \nabla_{\Gamma}+\sum_{k \geq 1} \varepsilon^{k} \mathcal{B}_{t}^{k}
$$

with

$$
\mathcal{B}_{\boldsymbol{t}}^{1}=\operatorname{div}_{\Gamma}(\boldsymbol{\theta} \cdot \boldsymbol{n})(2 \mathcal{R}-\mathcal{H}) \nabla_{\Gamma}+\mathcal{H} \operatorname{div}_{\Gamma}(\boldsymbol{\theta} \cdot \boldsymbol{n}) \nabla_{\Gamma}
$$

and

$$
\mathcal{B}_{\boldsymbol{t}}^{2}=\operatorname{div}_{\Gamma}(\boldsymbol{\theta} \cdot \boldsymbol{n})\left(2 \mathcal{R}^{2}-\mathcal{H} \mathcal{R}\right) \nabla_{\Gamma}+\mathcal{H} \operatorname{div}_{\Gamma}(\boldsymbol{\theta} \cdot \boldsymbol{n})(2 \mathcal{R}-\mathcal{H}) \nabla_{\Gamma}+\left(\mathcal{H}^{2}-\mathcal{G}\right) \operatorname{div}_{\Gamma}(\boldsymbol{\theta} \cdot \boldsymbol{n}) \nabla_{\Gamma}
$$


The transmission problem 4.1 can be rewritten as follows:

$$
\left\{\begin{array}{rlrl}
\sum_{\ell \geq 0} \varepsilon^{\ell}\left(\Delta \dot{u}_{e x t}^{\ell}+\kappa_{e}^{2} \dot{u}_{e x t}^{\ell}\right)= & 0 & \text { in } \Omega_{e x t} \\
\sum_{\ell \geq 0} \varepsilon^{\ell} \partial_{S}^{2} \mathbf{U}_{i n t}^{\ell} & = & & \\
& & -\sum_{\ell \geq 1} \varepsilon^{\ell} \Lambda_{1} \mathbf{U}_{i n t}^{\ell-1}-\sum_{\ell \geq 2} \varepsilon^{\ell} \Lambda_{k} \mathbf{U}_{i n t}^{\ell-k}\left(\Lambda_{2}+\kappa_{i}^{2}\right) \mathbf{U}_{i n t}^{\ell-2} & \text { in } \Gamma \times(0,1) \\
\sum_{\ell \geq 0} \varepsilon^{\ell} \mathbf{U}_{i n t}^{\ell} & & \sum_{\ell \geq 0} \varepsilon^{\ell} u_{e x t}^{\ell} \\
& -(\boldsymbol{\theta} \cdot \boldsymbol{n})\left(\frac{1}{\rho}-1\right)\left(\partial_{\boldsymbol{n}}\left(u_{e x t}^{0}+u^{i n c}\right)+\sum_{\ell \geq 1} \varepsilon^{\ell} \partial_{\boldsymbol{n}} u_{e x t}^{\ell}\right) & \text { on } \Gamma \times\{0\} \\
= & -\frac{1}{\rho} \sum_{\ell \geq 1} \varepsilon^{\ell} \partial_{\boldsymbol{n}} u_{\text {ext }}^{\ell-1} & \\
\sum_{\ell \geq 0} \varepsilon^{\ell} \partial_{S} \mathbf{U}_{i n t}^{\ell} & -\varepsilon\left[\left(1-\frac{1}{\rho}\right) \operatorname{div}_{\Gamma}(\boldsymbol{\theta} \cdot \boldsymbol{n}) \nabla_{\Gamma}+(\boldsymbol{\theta} \cdot \boldsymbol{n})\left(\kappa_{i}^{2}-\frac{1}{\rho} \kappa_{e}^{2}\right)\right]\left(u^{i n c}+u_{e x t}^{0}\right) & \\
& -\left[\left(1-\frac{1}{\rho}\right) \operatorname{div}_{\Gamma}(\boldsymbol{\theta} \cdot \boldsymbol{n}) \nabla_{\Gamma}+(\boldsymbol{\theta} \cdot \boldsymbol{n})\left(\kappa_{i}^{2}-\frac{1}{\rho} \kappa_{e}^{2}\right)\right] \sum_{\ell \geq 2} \varepsilon^{\ell} u_{e x t}^{\ell-1} & \text { on } \Gamma \times\{0\}
\end{array}\right.
$$

with either the Dirichlet condition on $\Gamma \times\{1\}$ that can be written

$$
\sum_{\ell \geq 1} \varepsilon^{\ell} \mathbf{U}_{i n t}^{\ell-1}(\cdot, 1)=(\boldsymbol{\theta} \cdot \boldsymbol{n}) \sum_{\ell \geq 0} \varepsilon^{\ell} \partial_{S} \mathbf{U}_{i n t}^{\ell}(\cdot, 1)
$$

or the Neumann condition on $\Gamma \times\{1\}$ that can be written

$$
\sum_{\ell \geq 0} \varepsilon^{\ell} \partial_{S} \mathbf{U}_{i n t}^{\ell}(\cdot, 1)=-\sum_{\ell \geq 1} \varepsilon^{\ell}\left[\operatorname{div}_{\Gamma}(\boldsymbol{\theta} \cdot \boldsymbol{n}) \nabla_{\Gamma}+(\boldsymbol{\theta} \cdot \boldsymbol{n}) \kappa_{i}^{2}\right] \mathbf{U}_{i n t}^{\ell-1}(\cdot, 1)-\sum_{\ell \geq 2} \varepsilon^{\ell} \mathcal{B}_{t}^{1} \mathbf{U}_{i n t}^{\ell-2}(\cdot, 1)+\cdots
$$

\subsection{Construction of the GIBC for the shape derivative}

The following two theorems give the GIBCs satisfied by the function $w_{[N]}^{\varepsilon}$, for $N=0,1,2$, which is an approximation of $\dot{u}_{e x t}^{\varepsilon}$ up to $O\left(\varepsilon^{N+1}\right)$.

Theorem 4.2. The GIBCs associated to the transmission problem characterising the first shape derivative of the solution in the Dirichlet case can be written for $N=0,1,2$ as follows

$$
w_{[N]}^{\varepsilon}+B^{\varepsilon, N} \partial_{\boldsymbol{n}} w_{[N]}^{\varepsilon}=S_{1}^{\varepsilon, N}\left(v_{[N]}^{\varepsilon}+u^{i n c}\right)+S_{2}^{\varepsilon, N} \partial_{\boldsymbol{n}}\left(v_{[N]}^{\varepsilon}+u^{i n c}\right)
$$

where

$$
\begin{gathered}
S_{1}^{\varepsilon, 0}=0 \quad \text { and } \quad S_{2}^{\varepsilon, 0}=-(\boldsymbol{\theta} \cdot \boldsymbol{n}) \mathrm{I} \\
S_{1}^{\varepsilon, 1}=\varepsilon\left[\left(1-\frac{1}{\rho}\right) \operatorname{div}_{\Gamma}(\boldsymbol{\theta} \cdot \boldsymbol{n}) \nabla_{\Gamma}+(\boldsymbol{\theta} \cdot \boldsymbol{n})\left(\kappa_{i}^{2}-\frac{1}{\rho} \kappa_{e}^{2}\right) \mathrm{I}\right] \quad \text { and } S_{2}^{\varepsilon, 1}=-(\boldsymbol{\theta} \cdot \boldsymbol{n})\left(1+\varepsilon \frac{1}{\rho} \mathcal{H}\right) \mathrm{I}
\end{gathered}
$$

and

$$
\begin{gathered}
S_{1}^{\varepsilon, 2}=\varepsilon\left(1+\frac{1}{2} \varepsilon \mathcal{H}\right)\left[\left(1-\frac{1}{\rho}\right) \operatorname{div}_{\Gamma}(\boldsymbol{\theta} \cdot \boldsymbol{n}) \nabla_{\Gamma}+(\boldsymbol{\theta} \cdot \boldsymbol{n})\left(\kappa_{i}^{2}-\frac{1}{\rho} \kappa_{e}^{2}\right) \mathrm{I}\right], \\
S_{2}^{\varepsilon, 2}=-(\boldsymbol{\theta} \cdot \boldsymbol{n})\left(1+\varepsilon \frac{1}{\rho} \mathcal{H}+\varepsilon^{2} \frac{1}{\rho}\left(\mathcal{H}^{2}-\mathcal{G}\right)\right) \mathrm{I}-\frac{1}{2 \rho} \varepsilon^{2}\left[\Delta_{\Gamma}+\kappa_{i}^{2} \mathrm{I}\right]((\boldsymbol{\theta} \cdot \boldsymbol{n}) \mathrm{I})-\frac{1}{2 \rho} \varepsilon^{2}(\boldsymbol{\theta} \cdot \boldsymbol{n})\left[\Delta_{\Gamma}+\kappa_{i}^{2} \mathrm{I}\right]
\end{gathered}
$$

Proof. When $\ell=0$, we obtain the following equations that can be split into two systems

$$
\left\{\begin{array}{rlll}
\partial_{S}^{2} \mathbf{U}_{i n t}^{0} & =0 & & \text { in } \Gamma \times(0,1) \\
\partial_{S} \mathbf{U}_{i n t}^{0} & =0 & & \text { on } \Gamma \times\{0\} \\
\mathbf{U}_{\text {int }}^{0} & =(\boldsymbol{\theta} \cdot \boldsymbol{n}) \partial_{S} \mathbf{U}_{\text {int }}^{1} & & \text { on } \Gamma \times\{1\}
\end{array}\right.
$$

and

We deduce

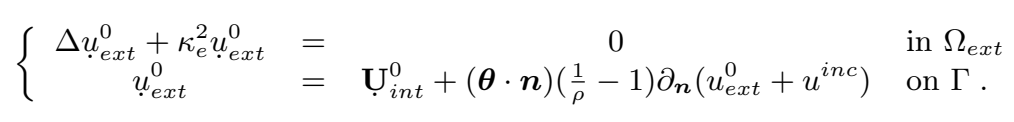

$$
\mathbf{U}_{i n t}^{0}(x, S)=-(\boldsymbol{\theta} \cdot \boldsymbol{n}) \frac{1}{\rho} \partial_{\boldsymbol{n}}\left(u_{\text {ext }}^{0}+u^{i n c}\right)
$$


and $u_{e x t}^{0}=-(\boldsymbol{\theta} \cdot \boldsymbol{n}) \partial_{\boldsymbol{n}}\left(u_{e x t}^{0}+u^{i n c}\right)$ on $\Gamma$. In this case we approach $\dot{u}_{e x t}^{\varepsilon}$ by the function $w_{[0]}^{\varepsilon}=u_{e x t}^{0}$ and then $\dot{u}_{e x t}^{\varepsilon}-w_{[0]}^{\varepsilon}=O(\varepsilon)$. When $\ell=1$, we obtain the two systems

$$
\left\{\begin{array}{rlrl}
\partial_{S}^{2} \mathbf{U}_{i n t}^{1}= & -\Lambda_{1} \mathbf{U}_{i n t}^{0}=0 & & \text { in } \Gamma \times(0,1) \\
\partial_{S} \mathbf{U}_{i n t}^{1}= & -\frac{1}{\rho} \partial_{\boldsymbol{n}} u_{\text {ext }}^{0} & \\
& -\left[\left(1-\frac{1}{\rho}\right) \operatorname{div}_{\Gamma}(\boldsymbol{\theta} \cdot \boldsymbol{n}) \nabla_{\Gamma}+(\boldsymbol{\theta} \cdot \boldsymbol{n})\left(\kappa_{i}^{2}-\frac{1}{\rho} \kappa_{e}^{2}\right)\right]\left(u_{e x t}^{0}+u^{i n c}\right) & & \text { on } \Gamma \times\{0\} \\
\mathbf{U}_{i n t}^{1}= & (\boldsymbol{\theta} \cdot \boldsymbol{n}) \partial_{S} \mathbf{U}_{i n t}^{2} & & \text { on } \Gamma \times\{1\} .
\end{array}\right.
$$

and

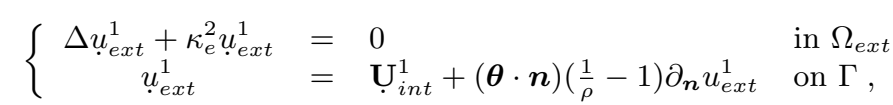

We compute $\mathbf{U}_{\text {int }}^{1}(\cdot, 1)=-(\boldsymbol{\theta} \cdot \boldsymbol{n}) \frac{1}{\rho}\left(\partial_{\boldsymbol{n}} u_{\text {ext }}^{1}+\mathcal{H} \partial_{\boldsymbol{n}}\left(u_{\text {ext }}^{0}+u^{i n c}\right)\right)$ and we deduce

$$
\begin{aligned}
\mathbf{U}_{i n t}^{1}(\cdot, S)= & -(S-1)\left[\frac{1}{\rho} \partial_{\boldsymbol{n}} u_{e x t \mid \Gamma}^{0}+\left[\left(1-\frac{1}{\rho}\right) \operatorname{div}_{\Gamma}(\boldsymbol{\theta} \cdot \boldsymbol{n}) \nabla_{\Gamma}+(\boldsymbol{\theta} \cdot \boldsymbol{n})\left(\kappa_{i}^{2}-\frac{1}{\rho} \kappa_{e}^{2}\right)\right]\left(u_{e x t}^{0}+u^{i n c}\right)\right] \\
& -(\boldsymbol{\theta} \cdot \boldsymbol{n}) \frac{1}{\rho}\left(\partial_{\boldsymbol{n}} u_{e x t}^{1}+\mathcal{H} \partial_{\boldsymbol{n}}\left(u_{e x t}^{0}+u^{i n c}\right)\right) .
\end{aligned}
$$

We have on $\Gamma$

$$
\begin{aligned}
u_{e x t}^{1}-\frac{1}{\rho} \partial_{\boldsymbol{n}} u_{e x t}^{0}= & -(\boldsymbol{\theta} \cdot \boldsymbol{n})\left(\partial_{\boldsymbol{n}} u_{e x t}^{1}+\mathcal{H} \frac{1}{\rho} \partial_{\boldsymbol{n}}\left(u_{e x t}^{0}+u^{i n c}\right)\right) \\
& +\left[\left(1-\frac{1}{\rho}\right) \operatorname{div}_{\Gamma}(\boldsymbol{\theta} \cdot \boldsymbol{n}) \nabla_{\Gamma}+(\boldsymbol{\theta} \cdot \boldsymbol{n})\left(\kappa_{i}^{2}-\frac{1}{\rho} \kappa_{e}^{2}\right)\right]\left(u_{e x t}^{0}+u^{i n c}\right) .
\end{aligned}
$$

and

$$
\begin{aligned}
\sum_{\ell=0}^{1} \varepsilon^{\ell} u_{e x t}^{\ell}-\frac{1}{\rho} \varepsilon \partial_{\boldsymbol{n}} u_{e x t}^{0}= & -(\boldsymbol{\theta} \cdot \boldsymbol{n})\left(\partial_{\boldsymbol{n}}\left(u^{i n c}+\sum_{\ell=0}^{1} \varepsilon^{\ell} u_{e x t}^{\ell}\right)+\mathcal{H} \frac{1}{\rho} \varepsilon \partial_{\boldsymbol{n}}\left(u_{e x t}^{0}+u^{i n c}\right)\right) \\
& +\varepsilon\left[\left(1-\frac{1}{\rho}\right) \operatorname{div}_{\Gamma}(\boldsymbol{\theta} \cdot \boldsymbol{n}) \nabla_{\Gamma}+(\boldsymbol{\theta} \cdot \boldsymbol{n})\left(\kappa_{i}^{2}-\frac{1}{\rho} \kappa_{e}^{2}\right)\right]\left(u_{e x t}^{0}+u^{i n c}\right) .
\end{aligned}
$$

In this case we approach $\dot{u}_{e x t}^{\varepsilon}$ by the function $w_{[1]}^{\varepsilon}$ which solves the Helmholtz equation and the boundary condition

$$
\begin{aligned}
w_{[1]}^{\varepsilon}+B^{\varepsilon, 1} \partial_{\boldsymbol{n}} w_{[1]}^{\varepsilon}= & -(\boldsymbol{\theta} \cdot \boldsymbol{n})\left(\partial_{\boldsymbol{n}}\left(u^{i n c}+v_{[1]}^{\varepsilon}\right)+\mathcal{H} \frac{1}{\rho} \varepsilon \partial_{\boldsymbol{n}}\left(v_{[1]}^{\varepsilon}+u^{i n c}\right)\right) \\
& +\varepsilon\left[\left(1-\frac{1}{\rho}\right) \operatorname{div}_{\Gamma}(\boldsymbol{\theta} \cdot \boldsymbol{n}) \nabla_{\Gamma}+(\boldsymbol{\theta} \cdot \boldsymbol{n})\left(\kappa_{i}^{2}-\frac{1}{\rho} \kappa_{e}^{2}\right)\right]\left(v_{[1]}^{\varepsilon}+u^{i n c}\right) .
\end{aligned}
$$

and then $\dot{u}_{e x t}^{\varepsilon}-w_{[1]}^{\varepsilon}=O\left(\varepsilon^{2}\right)$ on $\Gamma$.

When $\ell=2$, we obtain the two systems

$$
\left\{\begin{array}{rlrl}
\partial_{S}^{2} \mathbf{U}_{i n t}^{2}= & -\Lambda_{1} \mathbf{U}_{i n t}^{1}-\left(\Lambda_{2}+\kappa_{i}^{2}\right) \mathbf{U}_{i n t}^{0} & \text { in } \Gamma \times(0,1) \\
\partial_{S} \mathbf{U}_{i n t}^{2}= & -\frac{1}{\rho} \partial_{\boldsymbol{n}} u_{\text {ext }}^{1} & \\
& -\left[\left(1-\frac{1}{\rho}\right) \operatorname{div}_{\Gamma}(\boldsymbol{\theta} \cdot \boldsymbol{n}) \nabla_{\Gamma}+(\boldsymbol{\theta} \cdot \boldsymbol{n})\left(\kappa_{i}^{2}-\frac{1}{\rho} \kappa_{e}^{2}\right)\right] u_{\text {ext }}^{1} & & \text { on } \Gamma \times\{0\} \\
\mathbf{U}_{\text {int }}^{2}= & (\boldsymbol{\theta} \cdot \boldsymbol{n}) \partial_{S} \mathbf{U}_{\text {int }}^{3} & \text { on } \Gamma \times\{1\} .
\end{array}\right.
$$

and

We compute

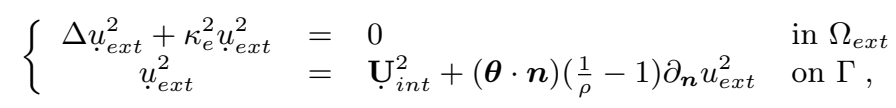

$$
\begin{aligned}
\partial_{S}^{2} \mathbf{U}_{i n t}^{2}(\cdot, S)= & -\mathcal{H}\left[\frac{1}{\rho} \partial_{\boldsymbol{n}} u_{\text {ext }}^{0}+\left[\left(1-\frac{1}{\rho}\right) \operatorname{div}_{\Gamma}(\boldsymbol{\theta} \cdot \boldsymbol{n}) \nabla_{\Gamma}+(\boldsymbol{\theta} \cdot \boldsymbol{n})\left(\kappa_{i}^{2}-\frac{1}{\rho} \kappa_{e}^{2}\right)\right]\left(u_{\text {ext }}^{0}+u^{i n c}\right)\right] \\
& +\left(\Delta_{\Gamma}+\kappa_{i}^{2}\right)(\boldsymbol{\theta} \cdot \boldsymbol{n}) \frac{1}{\rho} \partial_{\boldsymbol{n}}\left(u_{\text {ext }}^{0}+u^{i n c}\right) \\
\mathbf{U}_{i n t}^{2}(\cdot, 1)= & -\frac{1}{2}(\boldsymbol{\theta} \cdot \boldsymbol{n})\left[\left(2 \mathcal{H}^{2}-2 \mathcal{G}\right) \frac{1}{\rho} \partial_{\boldsymbol{n}}\left(u_{e x t}^{0}+u^{i n c}\right)+\left(\Delta_{\Gamma}+\kappa_{i}^{2}\right) \frac{1}{\rho} \partial_{\boldsymbol{n}}\left(u_{e x t}^{0}+u^{i n c}\right)\right] \\
& -(\boldsymbol{\theta} \cdot \boldsymbol{n}) \mathcal{H} \frac{1}{\rho} \partial_{\boldsymbol{n}} u_{\text {ext }}^{1}-(\boldsymbol{\theta} \cdot \boldsymbol{n}) \frac{1}{\rho} \partial_{\boldsymbol{n}} u_{\text {ext }}^{2} .
\end{aligned}
$$


and we conclude

$$
\begin{aligned}
\mathbf{U}_{i n t}^{2}(\cdot, S)= & -\left(\frac{S^{2}-1}{2}\right) \mathcal{H}\left[\frac{1}{\rho} \partial_{\boldsymbol{n}} u_{e x t}^{0}+\left[\left(1-\frac{1}{\rho}\right) \operatorname{div}_{\Gamma}(\boldsymbol{\theta} \cdot \boldsymbol{n}) \nabla_{\Gamma}+(\boldsymbol{\theta} \cdot \boldsymbol{n})\left(\kappa_{i}^{2}-\frac{1}{\rho} \kappa_{e}^{2}\right)\right]\left(u_{\text {ext }}^{0}+u^{i n c}\right)\right] \\
& +\left(\frac{S^{2}-1}{2}\right)\left(\Delta_{\Gamma}+\kappa_{i}^{2}\right)(\boldsymbol{\theta} \cdot \boldsymbol{n}) \frac{1}{\rho} \partial_{\boldsymbol{n}}\left(u_{e x t}^{0}+u^{i n c}\right) \\
& +(S-1)\left[-\frac{1}{\rho} \partial_{\boldsymbol{n}} u_{e x t}^{1}-\left[\left(1-\frac{1}{\rho}\right) \operatorname{div}_{\Gamma}(\boldsymbol{\theta} \cdot \boldsymbol{n}) \nabla_{\Gamma}+(\boldsymbol{\theta} \cdot \boldsymbol{n})\left(\kappa_{i}^{2}-\frac{1}{\rho} \kappa_{e}^{2}\right)\right] u_{e x t}^{1}\right] \\
& -\frac{1}{2}(\boldsymbol{\theta} \cdot \boldsymbol{n})\left[\left(2 \mathcal{H}^{2}-2 \mathcal{G}\right) \frac{1}{\rho} \partial_{\boldsymbol{n}}\left(u_{\text {ext }}^{0}+u^{i n c}\right)+\left(\Delta_{\Gamma}+\kappa_{i}^{2}\right) \frac{1}{\rho} \partial_{\boldsymbol{n}}\left(u_{e x t}^{0}+u^{i n c}\right)\right] \\
& -(\boldsymbol{\theta} \cdot \boldsymbol{n}) \mathcal{H} \frac{1}{\rho} \partial_{\boldsymbol{n}} u_{\text {ext }}^{1}-(\boldsymbol{\theta} \cdot \boldsymbol{n}) \frac{1}{\rho} \partial_{\boldsymbol{n}} u_{\text {ext }}^{2} .
\end{aligned}
$$

We have on $\Gamma$

$$
\begin{aligned}
& u_{\text {ext }}^{2}-\frac{1}{\rho} \partial_{\boldsymbol{n}} u_{\text {ext }}^{1}-\frac{1}{2} \frac{1}{\rho} \mathcal{H} \partial_{\boldsymbol{n}} u_{\text {ext }}^{0} \\
= & -(\boldsymbol{\theta} \cdot \boldsymbol{n})\left(\partial_{\boldsymbol{n}} u_{\text {ext }}^{2}+\mathcal{H} \frac{1}{\rho} \partial_{\boldsymbol{n}} u_{\text {ext }}^{1}\right) \\
& +\left[\left(1-\frac{1}{\rho}\right) \operatorname{div}_{\Gamma}(\boldsymbol{\theta} \cdot \boldsymbol{n}) \nabla_{\Gamma}+(\boldsymbol{\theta} \cdot \boldsymbol{n})\left(\kappa_{i}^{2}-\frac{1}{\rho} \kappa_{e}^{2}\right)\right] u_{\text {ext }}^{1} \\
& -\frac{1}{2}\left[\Delta_{\Gamma}+\kappa_{i}^{2}\right](\boldsymbol{\theta} \cdot \boldsymbol{n}) \frac{1}{\rho} \partial_{\boldsymbol{n}}\left(u_{\text {ext }}^{0}+u^{i n c}\right)-\frac{1}{2}(\boldsymbol{\theta} \cdot \boldsymbol{n})\left[\Delta_{\Gamma}+\kappa_{i}^{2}\right] \frac{1}{\rho} \partial_{\boldsymbol{n}}\left(u_{\text {ext }}^{0}+u^{i n c}\right) \\
& +\frac{1}{2} \mathcal{H}\left[\left(1-\frac{1}{\rho}\right) \operatorname{div}_{\Gamma}(\boldsymbol{\theta} \cdot \boldsymbol{n}) \nabla_{\Gamma}+(\boldsymbol{\theta} \cdot \boldsymbol{n})\left(\kappa_{i}^{2}-\frac{1}{\rho} \kappa_{e}^{2}\right)\right]\left(u_{\text {ext }}^{0}+u^{i n c}\right) \\
& -(\boldsymbol{\theta} \cdot \boldsymbol{n})\left(\mathcal{H}^{2}-\mathcal{G}\right) \frac{1}{\rho} \partial_{\boldsymbol{n}}\left(u_{\text {ext }}^{0}+u^{i n c}\right) \quad \text { on } \Gamma .
\end{aligned}
$$

and

$$
\begin{aligned}
& \sum_{\ell=0}^{2} \varepsilon^{\ell} u_{e x t}^{\ell}-\varepsilon \frac{1}{\rho} \partial_{\boldsymbol{n}} \sum_{\ell=0}^{2} \varepsilon^{\ell} u_{\text {ext }}^{\ell}-\frac{1}{2} \frac{1}{\rho} \mathcal{H} \partial_{\boldsymbol{n}} u_{\text {ext }}^{0} \\
= & -(\boldsymbol{\theta} \cdot \boldsymbol{n})\left(\partial_{\boldsymbol{n}}\left(u^{i n c}+\sum_{\ell=0}^{2} \varepsilon^{\ell} u_{e x t}^{\ell}\right)+\varepsilon \mathcal{H} \frac{1}{\rho} \partial_{\boldsymbol{n}}\left(u^{i n c}+\sum_{\ell=0}^{1} \varepsilon^{\ell} u_{e x t}^{\ell}\right)\right) \\
& +\varepsilon\left[\left(1-\frac{1}{\rho}\right) \operatorname{div}_{\Gamma}(\boldsymbol{\theta} \cdot \boldsymbol{n}) \nabla_{\Gamma}+(\boldsymbol{\theta} \cdot \boldsymbol{n})\left(\kappa_{i}^{2}-\frac{1}{\rho} \kappa_{e}^{2}\right)\right]\left(u^{i n c}+\sum_{\ell=0}^{1} \varepsilon^{\ell} u_{\text {ext }}^{\ell}\right) \\
& -\frac{1}{2} \varepsilon^{2}\left[\Delta_{\Gamma}+\kappa_{i}^{2}\right](\boldsymbol{\theta} \cdot \boldsymbol{n}) \frac{1}{\rho} \partial_{\boldsymbol{n}}\left(u_{\text {ext }}^{0}+u^{i n c}\right)-\frac{1}{2} \varepsilon^{2}(\boldsymbol{\theta} \cdot \boldsymbol{n})\left[\Delta_{\Gamma}+\kappa_{i}^{2}\right] \frac{1}{\rho} \partial_{\boldsymbol{n}}\left(u_{\text {ext }}^{0}+u^{i n c}\right) \\
& +\frac{1}{2} \varepsilon^{2} \mathcal{H}\left[\left(1-\frac{1}{\rho}\right) \operatorname{div} \Gamma(\boldsymbol{\theta} \cdot \boldsymbol{n}) \nabla_{\Gamma}+(\boldsymbol{\theta} \cdot \boldsymbol{n})\left(\kappa_{i}^{2}-\frac{1}{\rho} \kappa_{e}^{2}\right)\right]\left(u_{e x t}^{0}+u^{i n c}\right) \\
& -\varepsilon^{2}(\boldsymbol{\theta} \cdot \boldsymbol{n})\left(\mathcal{H}^{2}-\mathcal{G}\right) \frac{1}{\rho} \partial_{\boldsymbol{n}}\left(u_{\text {ext }}^{0}+u^{i n c}\right) \quad \text { on } \Gamma .
\end{aligned}
$$

In this case we approach $\dot{u}_{e x t}^{\varepsilon}$ by the function $w_{[2]}^{\varepsilon}$ which solves the Helmholtz equation and the boundary condition

$$
\begin{aligned}
w_{[2]}^{\varepsilon}+B^{\varepsilon, 2} \partial_{\boldsymbol{n}} w_{[2]}^{\varepsilon}= & -(\boldsymbol{\theta} \cdot \boldsymbol{n})\left(\partial_{\boldsymbol{n}}\left(u^{i n c}+v_{[2]}^{\varepsilon}\right)+\mathcal{H} \frac{1}{\rho} \varepsilon \partial_{\boldsymbol{n}}\left(v_{[2]}^{\varepsilon}+u^{i n c}\right)\right) \\
& +\varepsilon\left[\left(1-\frac{1}{\rho}\right) \operatorname{div}_{\Gamma}(\boldsymbol{\theta} \cdot \boldsymbol{n}) \nabla_{\Gamma}+(\boldsymbol{\theta} \cdot \boldsymbol{n})\left(\kappa_{i}^{2}-\frac{1}{\rho} \kappa_{e}^{2}\right)\right]\left(v_{[2]}^{\varepsilon}+u^{i n c}\right) \\
& -\frac{1}{2} \varepsilon^{2}\left[\Delta_{\Gamma}+\kappa_{i}^{2}\right](\boldsymbol{\theta} \cdot \boldsymbol{n}) \frac{1}{\rho} \partial_{\boldsymbol{n}}\left(u_{e x t}^{0}+u^{i n c}\right)-\frac{1}{2} \varepsilon^{2}(\boldsymbol{\theta} \cdot \boldsymbol{n})\left[\Delta_{\Gamma}+\kappa_{i}^{2}\right] \frac{1}{\rho} \partial_{\boldsymbol{n}}\left(v_{[2]}^{\varepsilon}+u^{i n c}\right) \\
& +\frac{1}{2} \varepsilon^{2} \mathcal{H}\left[\left(1-\frac{1}{\rho}\right) \operatorname{div}_{\Gamma}(\boldsymbol{\theta} \cdot \boldsymbol{n}) \nabla_{\Gamma}+(\boldsymbol{\theta} \cdot \boldsymbol{n})\left(\kappa_{i}^{2}-\frac{1}{\rho} \kappa_{e}^{2}\right)\right]\left(v_{[2]}^{\varepsilon}+u^{i n c}\right) \\
& -\varepsilon^{2}(\boldsymbol{\theta} \cdot \boldsymbol{n})\left(\mathcal{H}^{2}-\mathcal{G}\right) \frac{1}{\rho} \partial_{\boldsymbol{n}}\left(v_{[2]}^{\varepsilon}+u^{i n c}\right) \quad \text { on } \Gamma .
\end{aligned}
$$

and then $\dot{u}_{e x t}^{\varepsilon}-w_{[2]}^{\varepsilon}=O\left(\varepsilon^{3}\right)$ on $\Gamma$.

Theorem 4.3. The GIBCs associated to the transmission problem characterising the first shape derivative of the solution in the Neumann case can be written for $N=0,1,2$ as follows

$$
\partial_{\boldsymbol{n}} w_{[N]}^{\varepsilon}+B^{\varepsilon, N} w_{[N]}^{\varepsilon}=S_{1}^{\varepsilon, N}\left(v_{[N]}^{\varepsilon}+u^{i n c}\right)+S_{2}^{\varepsilon, N} \partial_{\boldsymbol{n}}\left(v_{[N]}^{\varepsilon}+u^{i n c}\right)
$$

where

$$
S_{1}^{\varepsilon, 0}=\left[\operatorname{div}_{\Gamma}(\boldsymbol{\theta} \cdot \boldsymbol{n}) \nabla_{\Gamma}+(\boldsymbol{\theta} \cdot \boldsymbol{n}) \kappa_{e}^{2} \mathrm{I}\right] \quad \text { and } \quad S_{2}^{\varepsilon, 0}=0,
$$

$S_{1}^{\varepsilon, 1}=\left[\operatorname{div}_{\Gamma}(\boldsymbol{\theta} \cdot \boldsymbol{n})(1+\rho \varepsilon(2 \mathcal{R}-\mathcal{H})) \nabla_{\Gamma}+(\boldsymbol{\theta} \cdot \boldsymbol{n}) \kappa_{e}^{2} \mathrm{I}\right]-(\boldsymbol{\theta} \cdot \boldsymbol{n}) \rho \varepsilon \mathcal{H} \kappa_{i}^{2} \mathrm{I}$ and $S_{2}^{\varepsilon, 1}=\varepsilon(1-\rho)\left[\Delta_{\Gamma}+\kappa_{i}^{2} \mathrm{I}\right]((\boldsymbol{\theta} \cdot \boldsymbol{n}) \mathrm{I})$ 
and

$$
\begin{gathered}
\left.S_{1}^{\varepsilon, 2}=\left[\operatorname{div}_{\Gamma}(\boldsymbol{\theta} \cdot \boldsymbol{n})\left(1+\rho \varepsilon(2 \mathcal{R}-\mathcal{H})+\rho \varepsilon^{2}\left(2 \mathcal{R}^{2}-\mathcal{H} \mathcal{R}\right]\right)\right) \nabla_{\Gamma}+(\boldsymbol{\theta} \cdot \boldsymbol{n}) \kappa_{e}^{2} \mathrm{I}\right]-(\boldsymbol{\theta} \cdot \boldsymbol{n}) \rho \varepsilon(\mathcal{H}-\varepsilon \mathcal{G}) \kappa_{i}^{2} \mathrm{I} \\
+\frac{\rho}{2} \varepsilon^{2}\left[\Delta_{\Gamma}+\kappa_{i}^{2} \mathrm{I}\right]\left[\operatorname{div}_{\Gamma}(\boldsymbol{\theta} \cdot \boldsymbol{n}) \nabla_{\Gamma}+(\boldsymbol{\theta} \cdot \boldsymbol{n}) \kappa_{i}^{2} \mathrm{I}\right]+\frac{\rho}{2} \varepsilon^{2}\left[\operatorname{div}_{\Gamma}(\boldsymbol{\theta} \cdot \boldsymbol{n}) \nabla_{\Gamma}+(\boldsymbol{\theta} \cdot \boldsymbol{n}) \kappa_{i}^{2} \mathrm{I}\right]\left[\Delta_{\Gamma}+\kappa_{i}^{2} \mathrm{I}\right], \\
S_{2}^{\varepsilon, 2}=\varepsilon(1-\rho)\left[\operatorname{div}_{\Gamma}\left(1+\varepsilon\left(\mathcal{R}-\frac{1}{2} \mathcal{H}\right)\right) \nabla_{\Gamma}+\left(1-\frac{1}{2} \varepsilon \mathcal{H}\right) \kappa_{i}^{2} \mathrm{I}\right]((\boldsymbol{\theta} \cdot \boldsymbol{n}) \mathrm{I}) .
\end{gathered}
$$

Proof. We detail the computations of the functions $u_{\text {ext }}^{\ell}$ only. The rank $\ell=0$ allows us to compute $\mathbf{U}_{\text {int }}^{0}$ only. We obtain the system

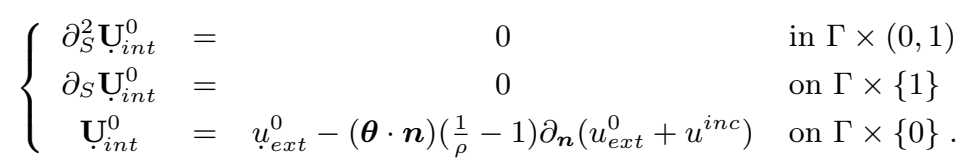

We conclude that

$$
\mathbf{U}_{i n t}^{0}(\cdot, S)=u_{e x t}^{0}-(\boldsymbol{\theta} \cdot \boldsymbol{n})\left(\frac{1}{\rho}-1\right) \partial_{\boldsymbol{n}}\left(u_{e x t}^{0}+u^{i n c}\right) .
$$

When $\ell=1$, we obtain the two systems

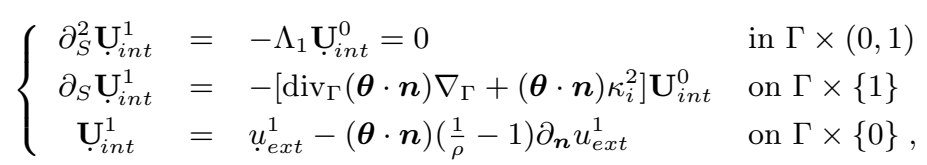

and

$$
\left\{\begin{array}{cccc}
\Delta u_{\text {ext }}^{1}+\kappa_{e}^{2} u_{\text {ext }}^{1} & = & 0 & \text { in } \Omega_{e x t} \\
\partial_{\boldsymbol{n}} u_{\text {ext }}^{1} & = & -\rho \partial_{S} \mathbf{U}_{\text {int }}^{1}+\left[(1-\rho) \operatorname{div}_{\Gamma}(\boldsymbol{\theta} \cdot \boldsymbol{n}) \nabla_{\Gamma}+(\boldsymbol{\theta} \cdot \boldsymbol{n})\left(\kappa_{e}^{2}-\rho \kappa_{i}^{2}\right)\right]\left(u_{e x t}^{0}+u^{i n c}\right) & \text { on } \Gamma .
\end{array}\right.
$$

We deduce

$$
\mathbf{U}_{i n t}^{1}(\cdot, S)=-S\left[\operatorname{div}_{\Gamma}(\boldsymbol{\theta} \cdot \boldsymbol{n}) \nabla_{\Gamma}+(\boldsymbol{\theta} \cdot \boldsymbol{n}) \kappa_{i}^{2}\right]\left(u_{e x t}^{0}+u^{i n c}\right)+u_{e x t}^{1}-(\boldsymbol{\theta} \cdot \boldsymbol{n})\left(\frac{1}{\rho}-1\right) \partial_{\boldsymbol{n}} u_{e x t}^{1} .
$$

and

$$
\partial_{\boldsymbol{n}} u_{\text {ext }}^{0}=\left[\operatorname{div}_{\Gamma}(\boldsymbol{\theta} \cdot \boldsymbol{n}) \nabla_{\Gamma}+(\boldsymbol{\theta} \cdot \boldsymbol{n}) \kappa_{e}^{2}\right]\left(u_{e x t}^{0}+u^{i n c}\right) \text { on } \Gamma .
$$

When $\ell=2$, we obtain the two systems

$$
\left\{\begin{array}{rlll}
\partial_{S}^{2} \mathbf{U}_{i n t}^{2}= & -\Lambda_{1} \mathbf{U}_{i n t}^{1}-\left(\Lambda_{2}+\kappa_{i}^{2}\right) \mathbf{U}_{i n t}^{0} & \text { in } \Gamma \times(0,1) \\
\partial_{S} \mathbf{U}_{i n t}^{2}= & -\left[\operatorname{div}_{\Gamma}(\boldsymbol{\theta} \cdot \boldsymbol{n}) \nabla_{\Gamma}+(\boldsymbol{\theta} \cdot \boldsymbol{n}) \kappa_{i}^{2}\right] \mathbf{U}_{i n t}^{1} & \\
& -\left[\operatorname{div}_{\Gamma}(\boldsymbol{\theta} \cdot \boldsymbol{n})(2 \mathcal{R}-\mathcal{H}) \nabla_{\Gamma}+\mathcal{H} \operatorname{div}_{\Gamma}(\boldsymbol{\theta} \cdot \boldsymbol{n}) \nabla_{\Gamma}\right] \mathbf{U}_{i n t}^{0} & & \text { on } \Gamma \times\{1\} \\
\mathbf{U}_{i n t}^{2}= & w_{e x t}^{2}-(\boldsymbol{\theta} \cdot \boldsymbol{n})\left(\frac{1}{\rho}-1\right) \partial_{\boldsymbol{n}} u_{e x t}^{2} & \text { on } \Gamma \times\{0\}
\end{array}\right.
$$

and

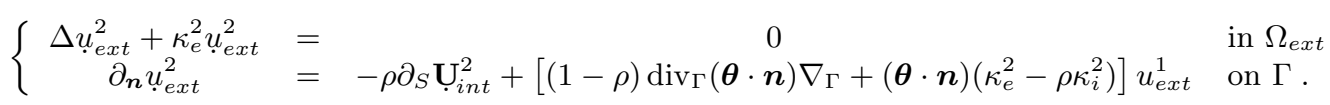

We compute

$$
\begin{aligned}
\partial_{S}^{2} \mathbf{U}_{i n t}^{2}(\cdot, S)= & -\mathcal{H}\left[\operatorname{div}_{\Gamma}(\boldsymbol{\theta} \cdot \boldsymbol{n}) \nabla_{\Gamma}+(\boldsymbol{\theta} \cdot \boldsymbol{n}) \kappa_{i}^{2}\right]\left(u_{e x t}^{0}+u^{i n c}\right) \\
& -\left(\Delta_{\Gamma}+\kappa_{i}^{2}\right)\left[u_{e x t}^{0}-(\boldsymbol{\theta} \cdot \boldsymbol{n})\left(\frac{1}{\rho}-1\right) \partial_{\boldsymbol{n}}\left(u_{e x t}^{0}+u^{i n c}\right)\right]
\end{aligned}
$$

and

$$
\begin{aligned}
\partial_{S} \mathbf{U}_{i n t}^{2}(\cdot, 1)= & -\left[\operatorname{div}_{\Gamma}(\boldsymbol{\theta} \cdot \boldsymbol{n}) \nabla_{\Gamma}+(\boldsymbol{\theta} \cdot \boldsymbol{n}) \kappa_{i}^{2}\right] u_{e x t}^{1} \\
& -\left[\operatorname{div}_{\Gamma}(\boldsymbol{\theta} \cdot \boldsymbol{n})(2 \mathcal{R}-\mathcal{H}) \nabla_{\Gamma}+\mathcal{H} \operatorname{div}_{\Gamma}(\boldsymbol{\theta} \cdot \boldsymbol{n}) \nabla_{\Gamma}\right]\left(u_{e x t}^{0}+u^{i n c}\right) .
\end{aligned}
$$

We deduce

$$
\begin{aligned}
\mathbf{U}_{i n t}^{2}(\cdot, S)= & -\left(\frac{S^{2}}{2}-S\right) \mathcal{H}\left[\operatorname{div}_{\Gamma}(\boldsymbol{\theta} \cdot \boldsymbol{n}) \nabla_{\Gamma}+(\boldsymbol{\theta} \cdot \boldsymbol{n}) \kappa_{i}^{2}\right]\left(u_{\text {ext }}^{0}+u^{i n c}\right) \\
& -\left(\frac{S^{2}}{2}-S\right)\left(\Delta_{\Gamma}+\kappa_{i}^{2}\right)\left(u_{\text {ext }}^{0}-(\boldsymbol{\theta} \cdot \boldsymbol{n})\left(\frac{1}{\rho}-1\right) \partial_{\boldsymbol{n}}\left(u_{\text {ext }}^{0}+u^{i n c}\right)\right) \\
& -S\left[\operatorname{div}_{\Gamma}(\boldsymbol{\theta} \cdot \boldsymbol{n}) \nabla_{\Gamma}+(\boldsymbol{\theta} \cdot \boldsymbol{n}) \kappa_{i}^{2}\right] u_{\text {ext }}^{1} \\
& -S\left[\operatorname{div}_{\Gamma}(\boldsymbol{\theta} \cdot \boldsymbol{n})(2 \mathcal{R}-\mathcal{H}) \nabla_{\Gamma}+\mathcal{H} \operatorname{div}_{\Gamma}(\boldsymbol{\theta} \cdot \boldsymbol{n}) \nabla_{\Gamma}\right]\left(u_{\text {ext }}^{0}+u^{i n c}\right) \\
& +u_{\text {ext }}^{2}(x)-(\boldsymbol{\theta} \cdot \boldsymbol{n})\left(\frac{1}{\rho}-1\right) \partial_{\boldsymbol{n}} u_{\text {ext }}^{2}
\end{aligned}
$$


and

$$
\begin{aligned}
\partial_{\boldsymbol{n}} u_{e x t}^{1}+\rho\left(\Delta_{\Gamma}+\kappa_{i}^{2}\right) u_{e x t \mid \Gamma}^{0}= & {\left[\operatorname{div}_{\Gamma}(\boldsymbol{\theta} \cdot \boldsymbol{n}) \nabla_{\Gamma}+(\boldsymbol{\theta} \cdot \boldsymbol{n}) \kappa_{e}^{2}\right] u_{e x t}^{1} } \\
& +\rho\left[\operatorname{div}_{\Gamma}(\boldsymbol{\theta} \cdot \boldsymbol{n})(2 \mathcal{R}-\mathcal{H}) \nabla_{\Gamma}-(\boldsymbol{\theta} \cdot \boldsymbol{n}) \mathcal{H} \kappa_{i}^{2}\right]\left(u_{e x t}^{0}+u^{i n c}\right) \\
& +\left(\Delta_{\Gamma}+\kappa_{i}^{2}\right)(\boldsymbol{\theta} \cdot \boldsymbol{n})(1-\rho) \partial_{\boldsymbol{n}}\left(u_{\text {ext }}^{0}+u^{i n c}\right)
\end{aligned}
$$

When $\ell=3$, we obtain the two systems

$$
\left\{\begin{array}{rlrl}
\partial_{S}^{2} \mathbf{U}_{\text {ext }}^{3}= & -\Lambda_{1} \mathbf{U}_{i n t}^{2}-\left(\Lambda_{2}+\kappa_{i}^{2}\right) \mathbf{U}_{i n t}^{1}(\cdot, S)-\Lambda_{3} \mathbf{U}_{i n t}^{0} & \text { in } \Gamma \times(0,1) \\
\partial_{S} \mathbf{U}_{i n t}^{3}= & -\left[\operatorname{div}_{\Gamma}(\boldsymbol{\theta} \cdot \boldsymbol{n}) \nabla_{\Gamma}+(\boldsymbol{\theta} \cdot \boldsymbol{n}) \kappa_{i}^{2}\right] \mathbf{U}_{\text {int }}^{2} \\
& -\left[\operatorname{div}_{\Gamma}(\boldsymbol{\theta} \cdot \boldsymbol{n})(2 \mathcal{R}-\mathcal{H}) \nabla_{\Gamma}+\mathcal{H} \operatorname{div}_{\Gamma}(\boldsymbol{\theta} \cdot \boldsymbol{n}) \nabla_{\Gamma}\right] \mathbf{U}_{i n t}^{1} & \\
& -\operatorname{div}_{\Gamma}(\boldsymbol{\theta} \cdot \boldsymbol{n})\left(2 \mathcal{R}^{2}-\mathcal{H} \mathcal{R}\right) \nabla_{\Gamma} \mathbf{U}_{i n t}^{0} & \\
& -\left[\mathcal{H} \operatorname{div}_{\Gamma}(\boldsymbol{\theta} \cdot \boldsymbol{n})(2 \mathcal{R}-\mathcal{H}) \nabla_{\Gamma}+\left(\mathcal{H}^{2}-\mathcal{G}\right) \operatorname{div}_{\Gamma}(\boldsymbol{\theta} \cdot \boldsymbol{n}) \nabla_{\Gamma}\right] \mathbf{U}_{i n t}^{0} & & \text { on } \Gamma \times\{1\} \\
\mathbf{U}_{\text {int }}^{3}= & u_{\text {ext }}^{3}-(\boldsymbol{\theta} \cdot \boldsymbol{n})\left(\frac{1}{\rho}-1\right) \partial_{\boldsymbol{n}} u_{\text {ext }}^{3} & \text { on } \Gamma \times\{0\},
\end{array}\right.
$$

and

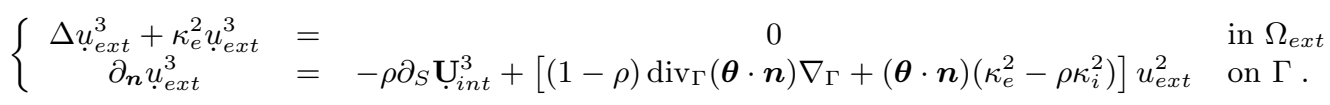

We deduce

$$
\begin{aligned}
\mathbf{U}_{i n t}^{3}(\cdot, S)= & -\left(\frac{S^{3}}{6}-\frac{S}{2}-\frac{S^{2}}{2}+S\right) \mathcal{H}\left[\mathcal{H}\left[\operatorname{div}_{\Gamma}(\boldsymbol{\theta} \cdot \boldsymbol{n}) \nabla_{\Gamma}+(\boldsymbol{\theta} \cdot \boldsymbol{n}) \kappa_{i}^{2}\right]\left(u_{e x t}^{0}+u^{i n c}\right)\right] \\
& -\left(\frac{S^{3}}{6}-\frac{S}{2}-\frac{S^{2}}{2}+S\right) \mathcal{H}\left[\left(\Delta_{\Gamma}+\kappa_{i}^{2}\right)\left(u_{\text {ext }}^{0}-(\boldsymbol{\theta} \cdot \boldsymbol{n})\left(\frac{1}{\rho}-1\right) \partial_{\boldsymbol{n}}\left(u_{e x t}^{0}+u^{i n c}\right)\right)\right] \\
& -\left(\frac{S^{2}}{2}-S\right) \mathcal{H}\left[\operatorname{div}_{\Gamma}(\boldsymbol{\theta} \cdot \boldsymbol{n}) \nabla_{\Gamma}+(\boldsymbol{\theta} \cdot \boldsymbol{n}) \kappa_{i}^{2}\right] u_{\text {ext }}^{1} \\
& -\left(\frac{S^{2}}{2}-S\right) \mathcal{H}\left[\operatorname{div}_{\Gamma}(\boldsymbol{\theta} \cdot \boldsymbol{n})(2 \mathcal{R}-\mathcal{H}) \nabla_{\Gamma}+\mathcal{H} \operatorname{div}_{\Gamma}(\boldsymbol{\theta} \cdot \boldsymbol{n}) \nabla_{\Gamma}\right]\left(u_{e x t}^{0}+u^{i n c}\right) \\
& +\left(\frac{S^{3}}{6}-\frac{S}{2}\right)\left(\Delta_{\Gamma}+\kappa_{i}^{2}\right)\left[\operatorname{div}_{\Gamma}(\boldsymbol{\theta} \cdot \boldsymbol{n}) \nabla_{\Gamma}+(\boldsymbol{\theta} \cdot \boldsymbol{n}) \kappa_{i}^{2}\right]\left(u_{e x t}^{0}+u^{i n c}\right) \\
& -\left(\frac{S^{2}}{2}-S\right)\left(\Delta_{\Gamma}+\kappa_{i}^{2}\right)\left[u_{e x t}^{1}(x)-(\boldsymbol{\theta} \cdot \boldsymbol{n})\left(\frac{1}{\rho}-1\right) \partial_{\boldsymbol{n}} u_{\text {ext }}^{1}\right] \\
& -\left(\frac{S^{3}}{6}-\frac{S}{2}\right)\left(\mathcal{H}^{2}-2 \mathcal{G}\right)\left[\operatorname{div}_{\Gamma}(\boldsymbol{\theta} \cdot \boldsymbol{n}) \nabla_{\Gamma}+(\boldsymbol{\theta} \cdot \boldsymbol{n}) \kappa_{i}^{2}\right]\left(u_{e x t}^{0}+u^{i n c}\right) \\
& -\left(\frac{S^{3}}{6}-\frac{S}{2}\right)\left(\operatorname{div}_{\Gamma}(2 \mathcal{R}-\mathcal{H}) \nabla_{\Gamma}+\mathcal{H} \Delta_{\Gamma}\right)\left[u_{\text {ext }}^{0}-(\boldsymbol{\theta} \cdot \boldsymbol{n})\left(\frac{1}{\rho}-1\right) \partial_{\boldsymbol{n}}\left(u_{\text {ext }}^{0}+u^{i n c}\right)\right] \\
& -S\left[\operatorname{div}_{\Gamma}(\boldsymbol{\theta} \cdot \boldsymbol{n}) \nabla_{\Gamma}+(\boldsymbol{\theta} \cdot \boldsymbol{n}) \kappa_{i}^{2}\right]\left(\frac{1}{2}\left(\Delta_{\Gamma}+\kappa_{i}^{2}\right)\left(u_{e x t}^{0}+u^{i n c}\right)+u_{e x t}^{2}\right) \\
& -S\left[\operatorname{div}_{\Gamma}(\boldsymbol{\theta} \cdot \boldsymbol{n})(2 \mathcal{R}-\mathcal{H}) \nabla_{\Gamma}+\mathcal{H} \operatorname{div}_{\Gamma}(\boldsymbol{\theta} \cdot \boldsymbol{n}) \nabla_{\Gamma}\right] u_{e x t}^{1}(x) \\
& -S \operatorname{div}_{\Gamma}(\boldsymbol{\theta} \cdot \boldsymbol{n})\left(2 \mathcal{R}^{2}-\mathcal{H} \mathcal{R}\right) \nabla_{\Gamma}\left(u_{\text {ext }}^{0}+u^{i n c}\right) \\
& -S\left[\mathcal{H} \operatorname{div}_{\Gamma}(\boldsymbol{\theta} \cdot \boldsymbol{n})(2 \mathcal{R}-\mathcal{H}) \nabla_{\Gamma}+\left(\mathcal{H}^{2}-\mathcal{G}\right) \operatorname{div}_{\Gamma}(\boldsymbol{\theta} \cdot \boldsymbol{n}) \nabla_{\Gamma}\right]\left(u_{e x t}^{0}+u^{i n c}\right) \\
& +u_{e x t}^{3}-(\boldsymbol{\theta} \cdot \boldsymbol{n})\left(\frac{1}{\rho}-1\right) \partial_{\boldsymbol{n}} u_{e x t}^{3} \cdot
\end{aligned}
$$

and

with

$$
\partial_{\boldsymbol{n}} u_{\text {ext }}^{2}+\rho\left(\Delta_{\Gamma}+\kappa_{i}^{2}\right) u_{\text {ext }}^{1}+\rho\left[\operatorname{div}_{\Gamma}\left(\mathcal{R}-\frac{1}{2} \mathcal{H}\right) \nabla_{\Gamma}-\frac{1}{2} \mathcal{H} \kappa_{i}^{2}\right] u_{\text {ext }}^{0}=f \quad \text { on } \Gamma
$$

$$
\begin{aligned}
f= & {\left[\operatorname{div}_{\Gamma}(\boldsymbol{\theta} \cdot \boldsymbol{n}) \nabla_{\Gamma}+(\boldsymbol{\theta} \cdot \boldsymbol{n}) \kappa_{e}^{2}\right] u_{e x t}^{2}+\rho\left[\operatorname{div}_{\Gamma}(\boldsymbol{\theta} \cdot \boldsymbol{n})(2 \mathcal{R}-\mathcal{H}) \nabla_{\Gamma}-(\boldsymbol{\theta} \cdot \boldsymbol{n}) \mathcal{H} \kappa_{i}^{2}\right] u_{e x t}^{1} } \\
& +\left(\Delta_{\Gamma}+\kappa_{i}^{2}\right)(\boldsymbol{\theta} \cdot \boldsymbol{n})(1-\rho) \partial_{\boldsymbol{n}} u_{\text {ext }}^{1}(x)+\rho \mathcal{G}(\boldsymbol{\theta} \cdot \boldsymbol{n}) \kappa_{i}^{2}\left(u_{e x t}^{0}+u^{i n c}\right) \\
& +\rho \operatorname{div}_{\Gamma}(\boldsymbol{\theta} \cdot \boldsymbol{n})\left(2 \mathcal{R}^{2}-\mathcal{H} \mathcal{R}\right) \nabla_{\Gamma}\left(u_{e x t}^{0}+u^{i n c}\right) \\
& +\left[\operatorname{div}_{\Gamma}\left(\mathcal{R}-\frac{1}{2} \mathcal{H}\right) \nabla_{\Gamma}-\frac{1}{2} \mathcal{H} \kappa_{i}^{2}\right](\boldsymbol{\theta} \cdot \boldsymbol{n})(1-\rho) \partial_{\boldsymbol{n}}\left(u_{e x t}^{0}+u^{i n c}\right) \\
& +\frac{1}{2} \rho\left(\Delta_{\Gamma}+\kappa_{i}^{2}\right)\left[\operatorname{div}_{\Gamma}(\boldsymbol{\theta} \cdot \boldsymbol{n}) \nabla_{\Gamma}+(\boldsymbol{\theta} \cdot \boldsymbol{n}) \kappa_{i}^{2}\right]\left(u_{e x t}^{0}+u^{i n c}\right) \\
& +\frac{1}{2} \rho\left[\operatorname{div}_{\Gamma}(\boldsymbol{\theta} \cdot \boldsymbol{n}) \nabla_{\Gamma}+(\boldsymbol{\theta} \cdot \boldsymbol{n}) \kappa_{i}^{2}\right]\left(\Delta_{\Gamma}+\kappa_{i}^{2}\right)\left(u_{e x t}^{0}+u^{i n c}\right)
\end{aligned}
$$




\section{Shape derivatives of the approximate solution}

This section is devoted to the shape derivative analysis of the approximate solution $v_{[N]}^{\varepsilon}$ of $u_{e x t}^{\varepsilon}$. It provides a second way to compute an approximation of the shape derivative of $u_{e x t}^{\varepsilon}$. It suffices to determine the exterior boundary value problem characterising the shape derivative $\dot{v}_{[N]}^{\varepsilon}$ of $v_{[N]}^{\varepsilon}$. Since $v_{[N]}^{\varepsilon} \simeq\left(u_{e x t}^{0}+u^{i n c}\right)+\sum_{\ell=1}^{N} \varepsilon^{\ell} u_{e x t}^{\ell}$, it is equivalent to determine, in a first step, the equations satisfied by every derivatives $\dot{u}_{e x t}^{\ell}$ of the functions occurring in the sum. In a second step, we obtain the equation satisfied by the desired approximation $\dot{v}_{[N]}^{\epsilon}$ for $N=0,1,2$ as in the proofs of Propositions 3.1 and 3.2. This is realized in the following two theorems.

Theorem 5.1. The boundary conditions characterising the first shape derivative of the approximate solution $v_{[N]}^{\varepsilon}$, for $N=0,1,2$, to the transmission problem with a Dirichlet condition on the interior boundary can be written as follows

where

$$
\dot{v}_{[N]}^{\varepsilon}+B^{\varepsilon, N} \partial_{\boldsymbol{n}} \dot{v}_{[N]}^{\varepsilon}=T_{1}^{\varepsilon, N}\left(v_{[N]}^{\varepsilon}+u^{i n c}\right)+T_{2}^{\varepsilon, N} \partial_{\boldsymbol{n}}\left(v_{[N]}^{\varepsilon}+u^{i n c}\right)
$$

$$
\begin{gathered}
T_{1}^{\varepsilon, 0}=0 \quad \text { and } \quad T_{2}^{\varepsilon, 0}=-(\boldsymbol{\theta} \cdot \boldsymbol{n}) \mathrm{I} \\
T_{1}^{\varepsilon, 1}=-\frac{1}{\rho} \varepsilon\left[\operatorname{div}_{\Gamma}(\boldsymbol{\theta} \cdot \boldsymbol{n}) \nabla_{\Gamma}+(\boldsymbol{\theta} \cdot \boldsymbol{n}) \kappa_{e}^{2} \mathrm{I}\right] \quad \text { and } \quad T_{2}^{\varepsilon, 1}=-(\boldsymbol{\theta} \cdot \boldsymbol{n})\left(1+\varepsilon \frac{1}{\rho} \mathcal{H}\right) \mathrm{I}
\end{gathered}
$$

and

$$
\begin{gathered}
T_{1}^{\varepsilon, 2}=-\frac{1}{\rho} \varepsilon\left(1+\frac{1}{2} \varepsilon \mathcal{H}\right)\left[\operatorname{div}_{\Gamma}(\boldsymbol{\theta} \cdot \boldsymbol{n}) \nabla_{\Gamma}+(\boldsymbol{\theta} \cdot \boldsymbol{n}) \kappa_{e}^{2} \mathrm{I}\right] \\
T_{2}^{\varepsilon, 2}=-(\boldsymbol{\theta} \cdot \boldsymbol{n})\left(1+\varepsilon \frac{1}{\rho} \mathcal{H}+\varepsilon^{2} \frac{1}{\rho}\left(\mathcal{H}^{2}-\mathcal{G}\right)\right) \mathrm{I}-\frac{1}{2 \rho} \varepsilon^{2}\left[\Delta_{\Gamma}(\boldsymbol{\theta} \cdot \boldsymbol{n})\right] \mathrm{I} .
\end{gathered}
$$

Proof. The result for the order $N=0$ is well-known [30, 22, 13]

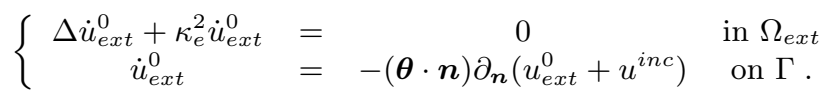

and using straightforward calculation we obtain the characterisation at the order $N=1$

$$
\Delta \dot{u}_{e x t}^{1}+\kappa_{e}^{2} \dot{u}_{e x t}^{1}=0, \quad \text { in } \Omega_{e x t}
$$

with, on $\Gamma$, the boundary condition

$$
\begin{aligned}
\dot{u}_{e x t}^{1}-\frac{1}{\rho} \partial_{\boldsymbol{n}} \dot{u}_{e x t}^{0}= & -(\boldsymbol{\theta} \cdot \boldsymbol{n})\left(\partial_{\boldsymbol{n}} u_{e x t}^{1}+\mathcal{H} \frac{1}{\rho} \partial_{\boldsymbol{n}}\left(u_{e x t}^{0}+u^{i n c}\right)\right) \\
& -\frac{1}{\rho}\left[\operatorname{div}_{\Gamma}(\boldsymbol{\theta} \cdot \boldsymbol{n}) \nabla_{\Gamma}+(\boldsymbol{\theta} \cdot \boldsymbol{n}) \kappa_{e}^{2} \mathrm{I}\right]\left(u_{e x t}^{0}+u^{i n c}\right) .
\end{aligned}
$$

Let us detail the computations for the order $N=2$ only. We know that the derivative $\dot{u}_{e x t}^{2}$ satisfies

$$
\Delta \dot{u}_{e x t}^{2}+\kappa_{e}^{2} \dot{u}_{e x t}^{2}=0, \text { in } \Omega_{e x t} .
$$

We essentially need to differentiate the boundary condition. We have for all $\boldsymbol{x} \in \Gamma$ and for all $\boldsymbol{\theta} \in \mathcal{O}$

$$
u_{e x t}^{2, \boldsymbol{\theta}}(\boldsymbol{x}+\boldsymbol{\theta}(\boldsymbol{x}))-\frac{1}{\rho} \partial_{\boldsymbol{n}_{\boldsymbol{\theta}}} u_{\text {ext }}^{1, \boldsymbol{\theta}}(\boldsymbol{x}+\boldsymbol{\theta}(\boldsymbol{x}))-\frac{1}{2} \frac{1}{\rho} \mathcal{H}_{\theta} \partial_{\boldsymbol{n}_{\boldsymbol{\theta}}}\left(u_{\text {ext }}^{0, \boldsymbol{\theta}}+u^{i n c}\right)(\boldsymbol{x}+\boldsymbol{\theta}(\boldsymbol{x}))=0 .
$$

We use $\dot{\mathcal{H}}=-\Delta_{\Gamma}(\theta \cdot \boldsymbol{n})=0[15$ and we obtain

$$
\begin{aligned}
0= & \dot{u}_{\text {ext }}^{2}+\boldsymbol{\theta} \cdot\left(\nabla u_{\text {ext }}^{2}\right)_{\Gamma}-\frac{1}{\rho} \partial_{\boldsymbol{n}} \dot{u}_{\text {ext }}^{1}+\frac{1}{\rho}\left[\nabla_{\Gamma} \boldsymbol{\theta} \cdot \boldsymbol{n}\right] \cdot \nabla_{\Gamma} u_{\text {ext }}^{1}-\frac{1}{\rho} \boldsymbol{\theta} \cdot \nabla\left(\partial_{\boldsymbol{n}} u_{\text {ext }}^{1}\right) \\
& -\frac{1}{2} \frac{1}{\rho} \mathcal{H} \partial_{\boldsymbol{n}} \dot{u}_{\text {ext }}^{0}+\frac{1}{2} \frac{1}{\rho} \Delta_{\Gamma}(\boldsymbol{\theta} \cdot \boldsymbol{n}) \partial_{\boldsymbol{n}}\left(u_{\text {ext }}^{0}+u^{i n c}\right)+\frac{1}{2} \frac{1}{\rho} \mathcal{H}\left[\nabla_{\Gamma} \boldsymbol{\theta} \cdot \boldsymbol{n}\right] \cdot \nabla_{\Gamma}\left(u_{\text {ext }}^{0}+u^{i n c}\right) \\
& -\frac{1}{2} \frac{1}{\rho} \theta \cdot \nabla\left(\mathcal{H} \partial_{\boldsymbol{n}}\left(u_{e x t}^{0}+u^{i n c}\right)\right) \\
= & \dot{u}_{\text {ext }}^{2}+(\boldsymbol{\theta} \cdot \boldsymbol{n}) \partial_{\boldsymbol{n}} u_{\text {ext }}^{2}-\frac{1}{\rho} \partial_{\boldsymbol{n}} \dot{u}_{\text {ext }}^{1}+\frac{1}{\rho}\left[\nabla_{\Gamma} \boldsymbol{\theta} \cdot \boldsymbol{n}\right] \cdot \nabla_{\Gamma} u_{\text {ext }}^{1}-\frac{1}{\rho}(\boldsymbol{\theta} \cdot \boldsymbol{n})\left(\partial_{\boldsymbol{n}}^{2} u_{\text {ext }}^{1}\right) \\
& -\frac{1}{2} \frac{1}{\rho} \mathcal{H} \partial_{\boldsymbol{n}} \dot{u}_{\text {ext }}^{0}+\frac{1}{2} \frac{1}{\rho} \Delta_{\Gamma}(\boldsymbol{\theta} \cdot \boldsymbol{n}) \partial_{\boldsymbol{n}}\left(u_{\text {ext }}^{0}+u^{i n c}\right)+\frac{1}{2} \frac{1}{\rho} \mathcal{H}\left[\nabla_{\Gamma} \boldsymbol{\theta} \cdot \boldsymbol{n}\right] \cdot \nabla_{\Gamma}\left(u_{\text {ext }}^{0}+u^{i n c}\right) \\
& -\frac{1}{2} \frac{1}{\rho}(\theta \cdot \boldsymbol{n})\left(\partial_{\boldsymbol{n}} \mathcal{H}\right) \partial_{\boldsymbol{n}}\left(u_{\text {ext }}^{0}+u^{i n c}\right)-\frac{1}{2} \frac{1}{\rho}(\theta \cdot \boldsymbol{n}) \mathcal{H} \partial_{\boldsymbol{n}}^{2}\left(u_{\text {ext }}^{0}+u^{i n c}\right)
\end{aligned}
$$

We use 27] $\partial_{n}^{2}=\Delta-\Delta_{\Gamma}-\mathcal{H} \partial_{n}$ and $\partial_{n} \mathcal{H}=-$ Trace $\left[\mathcal{R}^{2}\right]=\left(\mathcal{H}^{2}-2 \mathcal{G}\right)$ to conclude

$$
\begin{aligned}
& \dot{u}_{\text {ext }}^{2}-\frac{1}{\rho} \partial_{\boldsymbol{n}} \dot{u}_{\text {ext }}^{1}-\frac{1}{2} \frac{1}{\rho} \mathcal{H} \partial_{\boldsymbol{n}} \dot{u}_{\text {ext }}^{0} \\
= & -(\boldsymbol{\theta} \cdot \boldsymbol{n})\left(\partial_{\boldsymbol{n}} u_{\text {ext }}^{2}+\mathcal{H} \frac{1}{\rho} \partial_{\boldsymbol{n}} u_{\text {ext }}^{1}\right)-\frac{1}{\rho}\left[\operatorname{div}_{\Gamma}(\boldsymbol{\theta} \cdot \boldsymbol{n}) \nabla_{\Gamma}+(\boldsymbol{\theta} \cdot \boldsymbol{n}) \kappa_{e}^{2} \mathrm{I}\right] u_{\text {ext }}^{1} \\
& -(\boldsymbol{\theta} \cdot \boldsymbol{n})\left(\mathcal{H}^{2}-\mathcal{G}\right) \frac{1}{\rho} \partial_{\boldsymbol{n}}\left(u_{\text {ext }}^{0}+u^{i n c}\right)-\frac{1}{2 \rho}\left[\Delta_{\Gamma}(\boldsymbol{\theta} \cdot \boldsymbol{n})\right] \partial_{\boldsymbol{n}}\left(u_{\text {ext }}^{0}+u^{i n c}\right) \\
& -\frac{1}{2 \rho} \mathcal{H}\left[\operatorname{div}_{\Gamma}(\boldsymbol{\theta} \cdot \boldsymbol{n}) \nabla_{\Gamma}+(\boldsymbol{\theta} \cdot \boldsymbol{n}) \kappa_{e}^{2} \mathrm{I}\right]\left(u_{e x t}^{0}+u^{i n c}\right) .
\end{aligned}
$$


Theorem 5.2. The boundary conditions characterising the first shape derivative of the approximate solution $v_{[N]}^{\varepsilon}$, for $N=0,1,2$, to the transmission problem with a Neumann condition on the interior boundary can be written as follows

$$
\begin{aligned}
& \partial_{\boldsymbol{n}} \dot{v}_{[N]}^{\varepsilon}+B^{\varepsilon, N} \dot{v}_{[N]}^{\varepsilon}=T_{1}^{\varepsilon, N}\left(v_{[N]}^{\varepsilon}+u^{i n c}\right)+T_{2}^{\varepsilon, N} \partial_{\boldsymbol{n}}\left(v_{[N]}^{\varepsilon}+u^{i n c}\right) \\
& T_{1}^{\varepsilon, 0}=\left[\operatorname{div}_{\Gamma}(\boldsymbol{\theta} \cdot \boldsymbol{n}) \nabla_{\Gamma}+(\boldsymbol{\theta} \cdot \boldsymbol{n}) \kappa_{e}^{2} \mathrm{I}\right] \quad \text { and } \quad T_{2}^{\varepsilon, 0}=0, \\
& T_{1}^{\varepsilon, 1}=\left[\operatorname{div}_{\Gamma}(\boldsymbol{\theta} \cdot \boldsymbol{n})(1+\rho \varepsilon(2 \mathcal{R}-\mathcal{H})) \nabla_{\Gamma}+(\boldsymbol{\theta} \cdot \boldsymbol{n}) \kappa_{e}^{2} \mathrm{I}\right]-(\boldsymbol{\theta} \cdot \boldsymbol{n}) \rho \varepsilon \mathcal{H} \kappa_{i}^{2} \mathrm{I} \text { and } T_{2}^{\varepsilon, 1}=-\rho \varepsilon\left[\Delta_{\Gamma}+\kappa_{i}^{2} \mathrm{I}\right]((\boldsymbol{\theta} \cdot \boldsymbol{n}) \mathrm{I}) \\
& \left.T_{1}^{\varepsilon, 2}=\left[\operatorname{div}_{\Gamma}(\boldsymbol{\theta} \cdot \boldsymbol{n})\left(1+\rho \varepsilon(2 \mathcal{R}-\mathcal{H})+\rho \varepsilon^{2}\left(2 \mathcal{R}^{2}-\mathcal{H R}\right]\right)\right) \nabla_{\Gamma}+(\boldsymbol{\theta} \cdot \boldsymbol{n}) \kappa_{e}^{2} \mathrm{I}\right]-(\boldsymbol{\theta} \cdot \boldsymbol{n}) \rho \varepsilon(\mathcal{H}-\varepsilon \mathcal{G}) \kappa_{i}^{2} \mathrm{I} \\
& +\frac{\rho}{2} \varepsilon^{2} \operatorname{div}_{\Gamma}\left(2 D_{\Gamma}^{2}(\boldsymbol{\theta} \cdot \boldsymbol{n})-\left[\Delta_{\Gamma}(\boldsymbol{\theta} \cdot \boldsymbol{n})\right] \mathrm{I}\right) \nabla_{\Gamma}-\frac{\rho}{2} \varepsilon^{2} \kappa_{i}^{2}\left[\Delta_{\Gamma}(\boldsymbol{\theta} \cdot \boldsymbol{n})\right] \mathrm{I}, \\
& T_{2}^{\varepsilon, 2}=-\rho \varepsilon\left[\operatorname{div}_{\Gamma}\left(1+\varepsilon\left(\mathcal{R}-\frac{1}{2} \mathcal{H}\right)\right) \nabla_{\Gamma}+\left(1-\frac{1}{2} \varepsilon \mathcal{H}\right) \kappa_{i}^{2} \mathrm{I}\right]((\boldsymbol{\theta} \cdot \boldsymbol{n}) \mathrm{I}) .
\end{aligned}
$$

Proof. The results for the order $N=0$ is well-known $22,31,13$,

$$
\left\{\begin{array}{cccc}
\Delta \dot{u}_{e x t}^{0}+\kappa_{e}^{2} \dot{u}_{e x t}^{0} & = & 0 & \text { in } \Omega_{e x t} \\
\partial_{\boldsymbol{n}} \dot{u}_{e x t}^{0} & = & \operatorname{div}_{\Gamma}(\boldsymbol{\theta} \cdot \boldsymbol{n}) \nabla_{\Gamma}\left(u_{e x t}^{0}+u^{i n c}\right)+\kappa_{e}^{2}(\boldsymbol{\theta} \cdot \boldsymbol{n})\left(u_{e x t}^{0}+u^{i n c}\right) & \text { on } \Gamma .
\end{array}\right.
$$

and the characterisation at the order $N=1$ is obtained in [15]. We have

$$
\Delta \dot{u}_{e x t}^{1}+\kappa_{e}^{2} \dot{u}_{e x t}^{1}=0, \text { in } \Omega_{e x t}
$$

with, on $\Gamma$, the boundary condition

$$
\begin{aligned}
\partial_{\boldsymbol{n}} \dot{u}_{\text {ext }}^{1}+\rho\left[\Delta_{\Gamma}+\kappa_{i}^{2} \mathrm{I}\right] \dot{u}_{e x t}^{0} & =\left[\operatorname{div}_{\Gamma}\left((\boldsymbol{\theta} \cdot \boldsymbol{n}) \nabla_{\Gamma}\right)+\kappa_{e}^{2}(\boldsymbol{\theta} \cdot \boldsymbol{n}) \mathrm{I}\right] u_{e x t}^{1} \\
& -\rho\left[\operatorname{div}_{\Gamma}(\boldsymbol{\theta} \cdot \boldsymbol{n})(\mathcal{H}-2 \mathcal{R}) \nabla_{\Gamma}+\kappa_{i}^{2}(\boldsymbol{\theta} \cdot \boldsymbol{n}) \mathcal{H} \mathrm{I}\right]\left(u_{e x t}^{0}+u^{i n c}\right) \\
& -\rho\left[\Delta_{\Gamma}\left((\boldsymbol{\theta} \cdot \boldsymbol{n}) \partial_{\boldsymbol{n}}\left(u_{e x t}^{0}+u^{i n c}\right)\right)+\kappa_{i}^{2}(\boldsymbol{\theta} \cdot \boldsymbol{n}) \partial_{\boldsymbol{n}}\left(u_{e x t}^{0}+u^{i n c}\right)\right] .
\end{aligned}
$$

Let us detail the computations for the order $N=2$ only. The shape derivative $\dot{u}_{\text {ext }}^{2}$ satisfies

$$
\Delta \dot{u}_{e x t}^{2}+\kappa_{e}^{2} \dot{u}_{e x t}^{2}=0, \text { in } \Omega_{e x t} .
$$

On the boundary $\Gamma$, some difficulties arise. We have for all $\boldsymbol{x} \in \Gamma$ and for all $\boldsymbol{\theta} \in \mathcal{O}$

$\partial_{\boldsymbol{n}_{\boldsymbol{\theta}}} u_{e x t}^{2, \boldsymbol{\theta}}(\boldsymbol{x}+\boldsymbol{\theta}(\boldsymbol{x}))+\rho\left[\Delta_{\Gamma_{\boldsymbol{\theta}}}+\kappa_{i}^{2} \mathrm{I}\right] u_{\text {ext }}^{1, \boldsymbol{\theta}}(\boldsymbol{x}+\boldsymbol{\theta}(\boldsymbol{x}))+\rho\left[\operatorname{div}_{\Gamma_{\boldsymbol{\theta}}}\left(\mathcal{R}_{\boldsymbol{\theta}}-\frac{1}{2} \mathcal{H}_{\theta} \mathrm{I}\right) \nabla_{\Gamma_{\boldsymbol{\theta}}}-\frac{1}{2} \mathcal{H}_{\boldsymbol{\theta}} \kappa_{i}^{2} \mathrm{I}\right]\left(u_{\text {ext }}^{0, \boldsymbol{\theta}}+u^{i n c}\right)(\boldsymbol{x}+\boldsymbol{\theta}(\boldsymbol{x}))=0$.

We focus on the shape derivative of the third boundary term. For any small real value $t$, let us denote

$$
v_{t}=\frac{\rho}{2} \operatorname{div}_{\Gamma_{t \boldsymbol{\theta}}}\left[\left(2 \mathcal{R}_{t \boldsymbol{\theta}}-\mathcal{H}_{t \theta} \mathrm{I}\right) \nabla_{\Gamma_{t \boldsymbol{\theta}}}\left(u_{e x t}^{0, t \boldsymbol{\theta}}+u^{i n c}\right)\right]
$$

and

$$
w_{t}=-\frac{\rho}{2} \kappa_{i}^{2} \mathcal{H}_{t \boldsymbol{\theta}}\left(u_{e x t}^{0, t \boldsymbol{\theta}}+u^{i n c}\right)
$$

Denote $z_{t}=u_{e x t}^{0, t \boldsymbol{\theta}}+u^{i n c}$ and $\dot{z}=\dot{u}_{\text {ext }}^{0}$. We take a test function $\phi \in \mathcal{D}(\Gamma)$ which is supposed to be the restriction on $\Gamma$ of a function $\Phi \in \mathcal{D}\left(\mathbb{R}^{3}\right)$ satisfying $\partial_{n} \Phi=0$. We are led to compute $\frac{d}{d t}\left[\int_{\Gamma_{t \boldsymbol{\theta}}}\left(v_{t}+\right.\right.$ $\left.\left.w_{t}\right) \phi d \sigma\right]_{\mid t=0}$.

$$
\begin{aligned}
\frac{d}{d t}\left[\int_{\Gamma_{t \boldsymbol{\theta}}} v_{t} \phi d \sigma\right]_{\mid t=0} & =-\frac{\rho}{2}\left[\frac{d}{d t}\left[\int_{\Gamma_{t \boldsymbol{\theta}}}\left[\left(2 \mathcal{R}_{t \boldsymbol{\theta}}-\mathcal{H}_{t \boldsymbol{\theta}} I\right) \nabla_{\Gamma_{t \boldsymbol{\theta}}} z_{t} . \nabla_{\Gamma_{t \boldsymbol{\theta}}} \phi\right] d \sigma\right]_{\mid t=0}\right. \\
& =-\frac{\rho}{2}[(I)+(I I)]
\end{aligned}
$$

where [28, Eq. (4.44), pp. 192]

$$
(I)=\int_{\Gamma} \frac{d}{d t}\left[\left(2 \mathcal{R}_{t \boldsymbol{\theta}}-\mathcal{H}_{t \boldsymbol{\theta}} I\right) \nabla_{\Gamma_{t \boldsymbol{\theta}}} z_{t}\right]_{\mid t=0} . \nabla_{\Gamma} \phi d \sigma
$$

and

$$
(I I)=\int_{\Gamma} \boldsymbol{\theta} \cdot \boldsymbol{n} \partial_{\boldsymbol{n}}\left[(2 \mathcal{R}-\mathcal{H} \mathrm{I}) \nabla_{\Gamma} z \cdot \nabla_{\Gamma} \phi\right] d \sigma+\int_{\Gamma} \boldsymbol{\theta} \cdot \boldsymbol{n} \mathcal{H}\left[(2 \mathcal{R}-\mathcal{H} \mathrm{I}) \cdot \nabla_{\Gamma} z \cdot \nabla_{\Gamma} \phi\right] d \sigma
$$


Concerning $(I)$, we get 16

$$
\begin{aligned}
(I)= & \left.\int_{\Gamma} \nabla_{\Gamma} \phi \cdot\left[-\left(2 D_{\Gamma}^{2}(\boldsymbol{\theta} \cdot \boldsymbol{n})\right)+\Delta_{\Gamma}(\boldsymbol{\theta} \cdot \boldsymbol{n}) \mathrm{I}\right) \nabla_{\Gamma} z\right] d \sigma \\
& +\int_{\Gamma} \nabla_{\Gamma} \phi \cdot\left[(2 \mathcal{R}-\mathcal{H} \mathrm{I})\left(\nabla_{\Gamma} \dot{z}+\partial_{\boldsymbol{n}} z \nabla_{\Gamma}(\boldsymbol{\theta} \cdot \boldsymbol{n})\right)\right] d \sigma
\end{aligned}
$$

Indeed, set $\mathcal{A}=(2 \mathcal{R}-\mathcal{H I})$ and $\mathcal{A}=\left(a_{i j}\right)_{1 \leq i, j \leq 3}$. We have [27, pp. 69]

$$
\left(\partial_{n} a_{i j}\right)_{1 \leq i, j \leq 3}=\operatorname{Trace}\left(\mathcal{R}^{2}\right) \mathrm{I}-2 \mathcal{R}^{2} .
$$

It comes that

$$
\begin{aligned}
\partial_{n}\left(\mathcal{A} \nabla_{\Gamma} z \cdot \nabla_{\Gamma} \phi\right)+ & \left.\mathcal{A H} \nabla_{\Gamma} z \cdot \nabla_{\Gamma} \phi\right)= \\
& \mathcal{A} \partial_{n}\left[\nabla_{\Gamma} z \cdot \nabla_{\Gamma} \phi\right]+\left[\operatorname{Trace}\left(\mathcal{R}^{2}\right) \mathrm{I}-2 \mathcal{R}^{2}+\mathcal{H}(2 \mathcal{R}-\mathcal{H I}) \cdot\right] \nabla_{\Gamma} z \cdot \nabla_{\Gamma} \phi \\
= & \mathcal{A} \nabla_{\Gamma} \partial_{n} z \cdot \nabla_{\Gamma} \phi+\left(\mathcal{H A}-2 \mathcal{R} \mathcal{A}\left[\nabla_{\Gamma} z\right) \cdot \nabla_{\Gamma} \phi\right]+\left[\operatorname{Trace}\left(\left(\mathcal{R}^{2}\right) \mathrm{I}-2 \mathcal{R}^{2}\right) \cdot\right] \nabla_{\Gamma} z \cdot \nabla_{\Gamma} \phi \\
= & (2 \mathcal{R}-\mathcal{H I}) \nabla_{\Gamma} \partial_{n} z \cdot \nabla_{\Gamma} \phi+\left[\left(\operatorname{Trace}\left(\mathcal{R}^{2}\right)-\mathcal{H}^{2}\right) \mathrm{I}-6 \mathcal{R}^{2}+4 \mathcal{H} \mathcal{R}\right] \nabla_{\Gamma} z \cdot \nabla_{\Gamma} \phi \\
= & (2 \mathcal{R}-\mathcal{H I}) \nabla_{\Gamma} \partial_{n} z \cdot \nabla_{\Gamma} \phi+\left[-2 \mathcal{G I}-6 \mathcal{R}^{2}+4 \mathcal{H R}\right] \nabla_{\Gamma} z \cdot \nabla_{\Gamma} \phi \\
= & (2 \mathcal{R}-\mathcal{H I}) \nabla_{\Gamma} \partial_{n} z \cdot \nabla_{\Gamma} \phi+\left[-4 \mathcal{R}^{2}+2 \mathcal{H R}\right] \nabla_{\Gamma} z \cdot \nabla_{\Gamma} \phi
\end{aligned}
$$

Finally, gathering all the terms we get

$$
\begin{aligned}
(I I) & =\int_{\Gamma} \phi \operatorname{div}_{\Gamma}\left[\boldsymbol{\theta} \cdot \boldsymbol{n}\left(4 \mathcal{R}^{2}-2 \mathcal{H R}\right) \nabla_{\Gamma} z\right] \\
& +\int_{\Gamma} \phi \operatorname{div}_{\Gamma}\left[(\boldsymbol{\theta} \cdot \boldsymbol{n})(\mathcal{H} I-2 \mathcal{R}) \nabla_{\Gamma}\left(\partial_{\boldsymbol{n}} z\right)\right]
\end{aligned}
$$

From $(I)+(I I)$ we obtain

$$
\begin{aligned}
\frac{d}{d t}\left[\int_{\Gamma_{t \boldsymbol{\theta}}} v_{t} \phi d \sigma\right]_{\mid t=0} & =-\frac{\rho}{2} \int_{\Gamma} \phi \operatorname{div}_{\Gamma}\left[\left(2 \mathrm{D}_{\Gamma}\left(\nabla_{\Gamma}(\boldsymbol{\theta} \cdot \boldsymbol{n})\right)-\Delta_{\Gamma}(\boldsymbol{\theta} \cdot \boldsymbol{n}) I\right) \nabla_{\Gamma} z\right] d \sigma \\
& -\frac{\rho}{2} \int_{\Gamma} \phi \operatorname{div}_{\Gamma}\left[(-2 \mathcal{R}+\mathcal{H} \mathrm{I}) \nabla_{\Gamma} \dot{z}\right] d \sigma \\
& -\rho \int_{\Gamma} \phi \operatorname{div}_{\Gamma}\left[\boldsymbol{\theta} \cdot \boldsymbol{n}\left(2 \mathcal{R}^{2}-\mathcal{H} \mathcal{R}\right) \nabla_{\Gamma} z\right] \\
& -\frac{\rho}{2} \int_{\Gamma} \phi \operatorname{div}_{\Gamma}\left[(\boldsymbol{\theta} \cdot \boldsymbol{n})(\mathcal{H} I-2 \mathcal{R}) \nabla_{\Gamma}\left(\partial_{\boldsymbol{n}} z\right)\right] \\
& -\frac{\rho}{2} \int_{\Gamma} \phi \cdot \operatorname{div}_{\Gamma}\left[(2 \mathcal{R}-\mathcal{H I})\left(\partial_{\boldsymbol{n}} z \nabla_{\Gamma}(\boldsymbol{\theta} \cdot \boldsymbol{n})\right)\right] d \sigma
\end{aligned}
$$

Following the same lines as before, we get the following

$$
\begin{aligned}
\frac{d}{d t}\left[\int_{\Gamma_{t \boldsymbol{\theta}}} w_{t} \phi d \sigma\right]_{\mid t=0} & =-\frac{\rho}{2} \int_{\Gamma} \mathcal{H} \kappa_{i}^{2} \dot{z} \phi d \sigma-\frac{\rho}{2} \kappa_{i}^{2} \int_{\Gamma}(\boldsymbol{\theta} \cdot \boldsymbol{n}) \mathcal{H}\left(\partial_{n} z+\mathcal{H} z\right) \phi d \sigma \\
& +\frac{\rho}{2} \kappa_{i}^{2}\left[\int_{\Gamma}\left(\Delta_{\Gamma}(\boldsymbol{\theta} \cdot \boldsymbol{n})+\boldsymbol{\theta} \cdot \boldsymbol{n}\left(\mathcal{H}^{2}-2 \mathcal{G}\right)\right) z \phi d \sigma\right]
\end{aligned}
$$

We finaly get on $\Gamma$

$$
\begin{aligned}
& \partial_{\boldsymbol{n}} \dot{u}_{\text {ext }}^{2}+\rho\left(\Delta_{\Gamma}+\kappa_{i}^{2}\right) \dot{u}_{\text {ext }}^{1}+\rho\left[\left(\operatorname{div}_{\Gamma}\left(\mathcal{R}-\frac{1}{2} \mathcal{H}\right) \nabla_{\Gamma}\right)-\frac{1}{2} \mathcal{H} \kappa_{i}^{2} \mathrm{I}\right] \dot{u}_{\text {ext }}^{0} \\
= & {\left[\operatorname{div}_{\Gamma} \boldsymbol{\theta} \cdot \boldsymbol{n} \nabla_{\Gamma}+\kappa_{e}^{2} \boldsymbol{\theta} \cdot \boldsymbol{n} \mathrm{I}\right] u_{e x t}^{2}-\rho\left[\operatorname{div}_{\Gamma} \boldsymbol{\theta} \cdot \boldsymbol{n}(\mathcal{H}-2 \mathcal{R}) \nabla_{\Gamma}+\kappa_{i}^{2} \boldsymbol{\theta} \cdot \boldsymbol{n} \mathcal{H} \mathrm{I}\right]\left(u_{e x t}^{1}+u^{i n c}\right) } \\
& -\rho\left[\Delta_{\Gamma}+\kappa_{i}^{2} \mathrm{I}\right](\boldsymbol{\theta} \cdot \boldsymbol{n}) \partial_{\boldsymbol{n}}\left(u_{e x t}^{1}+u^{i n c}\right)+\frac{\rho}{2} \operatorname{div}_{\Gamma}\left[\left(2 \mathrm{D}_{\Gamma}^{2}(\boldsymbol{\theta} \cdot \boldsymbol{n})-\left[\Delta_{\Gamma}(\boldsymbol{\theta} \cdot \boldsymbol{n})\right] \mathrm{I}\right) \nabla_{\Gamma}\left(u_{e x t}^{0}+u^{i n c}\right)\right] \\
& +\rho \operatorname{div}_{\Gamma}\left[\boldsymbol{\theta} \cdot \boldsymbol{n}\left(2 \mathcal{R}^{2}-\mathcal{H} \mathcal{R}\right) \nabla_{\Gamma}\left(u_{e x t}^{0}+u^{i n c}\right)\right]-\frac{\rho}{2} \kappa_{i}^{2}\left[\left(\Delta_{\Gamma}(\boldsymbol{\theta} \cdot \boldsymbol{n})-2 \boldsymbol{\theta} \cdot \boldsymbol{n} \mathcal{G} \mathrm{I}\right)\left(u_{e x t}^{0}+u^{i n c}\right)\right] \\
& +\frac{\rho}{2} \operatorname{div}_{\Gamma}\left[(\mathcal{H I}-2 \mathcal{R}) \nabla_{\Gamma}\left(\boldsymbol{\theta} \cdot \boldsymbol{n} \partial_{\boldsymbol{n}}\left(u_{e x t}^{0}+u^{i n c}\right)\right)\right]+\frac{\rho}{2} \kappa_{i}^{2} \boldsymbol{\theta} \cdot \boldsymbol{n} \mathcal{H} \partial_{n}\left(u_{e x t}^{0}+u^{i n c}\right)
\end{aligned}
$$




\section{Comparison of the two approaches}

In this section, we focus on the remainder $r_{[N]}^{\varepsilon}=\dot{v}_{[N]}^{\varepsilon}-w_{[N]}^{\varepsilon}$ for $N=0,1,2$. Our main result is the following. Theorem 6.1. Let $N=0,1,2$. There exists some constants $C_{\Gamma}, C_{R}$ and $C_{\infty}$ independant on $\varepsilon$ such that

$$
\begin{aligned}
\left\|r_{[N]}^{\varepsilon}\right\|_{H^{\frac{1}{2}}(\Gamma)} & \leq C_{\Gamma} \varepsilon^{N+1}, \\
\left\|r_{[N]}^{\varepsilon}\right\|_{H^{1}\left(\Omega_{e x t} \cap B_{R}\right)} & \leq C_{R} \varepsilon^{N+1}, \\
\left\|r_{\infty,[N]}^{\varepsilon}\right\|_{L^{2}\left(\mathbb{S}^{2}\right)} & \leq C_{\infty} \varepsilon^{N+1},
\end{aligned}
$$

where $R$ is sufficiently large such that $\Omega \subset B_{R}$.

It is sufficient to prove the results for $N=2$. It relies on the fact that the difference between the right-hand sides $\left(F_{[N]}^{\varepsilon, 1}-F_{|N|}^{\varepsilon, 2}\right)$ is up to $O\left(\varepsilon^{N+1}\right)$. Then, the theory presented in [12] allows us to deduce the above estimates. We needs the following two propositions.

Proposition 6.2. We have

$$
\begin{aligned}
& \frac{1}{2} \operatorname{div}_{\Gamma}\left[\left(2 D_{\Gamma}^{2}(\boldsymbol{\theta} \cdot \boldsymbol{n})-\Delta_{\Gamma}(\boldsymbol{\theta} \cdot \boldsymbol{n}) \mathrm{I}\right) \nabla_{\Gamma} u\right]-\frac{1}{2} \kappa_{i}^{2}\left[u \Delta_{\Gamma}(\boldsymbol{\theta} \cdot \boldsymbol{n})\right] \\
- & \frac{1}{2}\left(\Delta_{\Gamma}+\kappa_{i}^{2}\right)\left[\operatorname{div}_{\Gamma}(\boldsymbol{\theta} \cdot \boldsymbol{n}) \nabla_{\Gamma}+(\boldsymbol{\theta} \cdot \boldsymbol{n}) \kappa_{i}^{2}\right] u-\frac{1}{2}\left[\operatorname{div}_{\Gamma}(\boldsymbol{\theta} \cdot \boldsymbol{n}) \nabla_{\Gamma}+(\boldsymbol{\theta} \cdot \boldsymbol{n}) \kappa_{i}^{2}\right]\left[\Delta_{\Gamma}+\kappa_{i}^{2}\right] u \\
+ & {\left[\Delta_{\Gamma}+\kappa_{i}^{2}\right]\left((\boldsymbol{\theta} \cdot \boldsymbol{n})\left(\Delta_{\Gamma} u+\kappa_{i}^{2} u\right)\right)=0 . }
\end{aligned}
$$

Proof. It is straightforward to verify that the summation of the coefficients in $\kappa_{i}^{2}$ and $\kappa_{i}^{4}$ vanish. Then we have

$$
\begin{aligned}
& \frac{1}{2} \operatorname{div}_{\Gamma}\left[\left(2 D_{\Gamma}^{2}(\boldsymbol{\theta} \cdot \boldsymbol{n})-\Delta_{\Gamma}(\boldsymbol{\theta} \cdot \boldsymbol{n}) \mathrm{I}\right) \nabla_{\Gamma} u\right]-\frac{1}{2} \kappa_{i}^{2}\left[u \Delta_{\Gamma}(\boldsymbol{\theta} \cdot \boldsymbol{n})\right] \\
& \left.-\frac{1}{2}\left(\Delta_{\Gamma}+\kappa_{i}^{2}\right)\left[\operatorname{div}_{\Gamma}(\boldsymbol{\theta} \cdot \boldsymbol{n}) \nabla_{\Gamma}+(\boldsymbol{\theta} \cdot \boldsymbol{n}) \kappa_{i}^{2}\right] u-\frac{1}{2}\left[\operatorname{div}_{\Gamma}(\boldsymbol{\theta} \cdot \boldsymbol{n}) \nabla_{\Gamma}+(\boldsymbol{\theta} \cdot \boldsymbol{n}) \kappa_{i}^{2}\right]\left(\Delta_{\Gamma}+\kappa_{i}^{2}\right) u\right) \\
& +\left[\Delta_{\Gamma}+\kappa_{i}^{2}\right]\left((\boldsymbol{\theta} \cdot \boldsymbol{n})\left(\Delta_{\Gamma} u+\kappa_{i}^{2} u\right)\right) \\
= & \frac{1}{2} \operatorname{div}_{\Gamma}\left[\left(2 D_{\Gamma}^{2}(\boldsymbol{\theta} \cdot \boldsymbol{n})-\Delta_{\Gamma}(\boldsymbol{\theta} \cdot \boldsymbol{n}) \mathrm{I}\right) \nabla_{\Gamma} u\right]-\frac{1}{2} \Delta_{\Gamma}\left(\operatorname{div}_{\Gamma}(\boldsymbol{\theta} \cdot \boldsymbol{n}) \nabla_{\Gamma} u\right) \\
& -\frac{1}{2}\left[\operatorname{div}_{\Gamma}(\boldsymbol{\theta} \cdot \boldsymbol{n}) \nabla_{\Gamma}\right]\left(\Delta_{\Gamma} u\right)+\Delta_{\Gamma}\left((\boldsymbol{\theta} \cdot \boldsymbol{n}) \Delta_{\Gamma} u\right) \\
= & \frac{1}{2} \operatorname{div}_{\Gamma}\left[\left(2 D_{\Gamma}^{2}(\boldsymbol{\theta} \cdot \boldsymbol{n})\right) \nabla_{\Gamma} u\right]-\frac{1}{2} \nabla_{\Gamma}\left(\Delta_{\Gamma}(\boldsymbol{\theta} \cdot \boldsymbol{n})\right) \cdot \nabla_{\Gamma} u-\frac{1}{2}\left(\Delta_{\Gamma}(\boldsymbol{\theta} \cdot \boldsymbol{n})\right)\left(\Delta_{\Gamma} u\right) \\
& -\frac{1}{2} \Delta_{\Gamma}\left(\nabla_{\Gamma}(\boldsymbol{\theta} \cdot \boldsymbol{n}) \cdot \nabla_{\Gamma} u\right)-\frac{1}{2}\left(\Delta_{\Gamma}(\boldsymbol{\theta} \cdot \boldsymbol{n})\right)\left(\Delta_{\Gamma} u\right)-\nabla_{\Gamma}(\boldsymbol{\theta} \cdot \boldsymbol{n}) \cdot \nabla_{\Gamma}\left(\Delta_{\Gamma} u\right)-\frac{1}{2}(\boldsymbol{\theta} \cdot \boldsymbol{n})\left(\Delta_{\Gamma}^{2} u\right) \\
& -\frac{1}{2} \nabla_{\Gamma}(\boldsymbol{\theta} \cdot \boldsymbol{n}) \cdot \nabla_{\Gamma}\left(\Delta_{\Gamma} u\right)-\frac{1}{2}(\boldsymbol{\theta} \cdot \boldsymbol{n})\left(\Delta_{\Gamma}^{2} u\right)+\left(\Delta_{\Gamma}(\boldsymbol{\theta} \cdot \boldsymbol{n})\right)\left(\Delta_{\Gamma} u\right)+2 \nabla_{\Gamma}(\boldsymbol{\theta} \cdot \boldsymbol{n}) \cdot \nabla_{\Gamma} \Delta_{\Gamma} u+(\boldsymbol{\theta} \cdot \boldsymbol{n})\left(\Delta_{\Gamma}^{2} u\right) \\
= & \operatorname{div}_{\Gamma}\left[D_{\Gamma}^{2}(\boldsymbol{\theta} \cdot \boldsymbol{n}) \nabla_{\Gamma} u\right]-\frac{1}{2} \nabla_{\Gamma} u \cdot \nabla_{\Gamma}\left(\Delta_{\Gamma}(\boldsymbol{\theta} \cdot \boldsymbol{n})\right)+\frac{1}{2} \nabla_{\Gamma}(\boldsymbol{\theta} \cdot \boldsymbol{n}) \cdot \nabla_{\Gamma}\left(\Delta_{\Gamma} u\right)-\frac{1}{2} \Delta_{\Gamma}\left[\nabla_{\Gamma} u \cdot \nabla_{\Gamma}(\boldsymbol{\theta} \cdot \boldsymbol{n})\right]
\end{aligned}
$$

We end the proof once we use the following two relations

$$
\operatorname{div}_{\Gamma}\left[D_{\Gamma}^{2}(\boldsymbol{\theta} \cdot \boldsymbol{n}) \nabla_{\Gamma} u\right]=\nabla_{\Gamma}\left[\Delta_{\Gamma}(\boldsymbol{\theta} \cdot \boldsymbol{n})\right] \cdot \nabla_{\Gamma} u+D_{\Gamma}^{2}(\boldsymbol{\theta} \cdot \boldsymbol{n}): D_{\Gamma}^{2} u
$$

where for two $(3 \times 3)$ matrices $A$ and $B$ whose columns are denoted by $\left(a_{1}, a_{2}, a_{3}\right)$ and $\left(b_{1}, b_{2}, b_{3}\right)$, respectively, we set $A: B=a_{1} \cdot b_{1}+a_{2} \cdot b_{2}+a_{3} \cdot b_{3}$ and,

$$
\frac{1}{2} \Delta_{\Gamma}\left[\nabla_{\Gamma} u \cdot \nabla_{\Gamma}(\boldsymbol{\theta} \cdot \boldsymbol{n})\right]=\frac{1}{2} \nabla_{\Gamma}(\boldsymbol{\theta} \cdot \boldsymbol{n}) \cdot \nabla_{\Gamma}\left(\Delta_{\Gamma} u\right)+\frac{1}{2} \nabla_{\Gamma} u \cdot \nabla_{\Gamma}\left(\Delta_{\Gamma}(\boldsymbol{\theta} \cdot \boldsymbol{n})\right)+D_{\Gamma}^{2}(\boldsymbol{\theta} \cdot \boldsymbol{n}): D_{\Gamma}^{2} u .
$$

The second result concerns the GIBC satisfied by $r_{[N]}^{\varepsilon}$ on $\Gamma$.

Lemma 6.3. In the Neuman case, we have

$$
\partial_{\boldsymbol{n}} r_{[2]}^{\varepsilon}+\rho \varepsilon\left(\Delta_{\Gamma}+\kappa_{i}^{2}\right) r_{[2]}^{\varepsilon}+\varepsilon^{2} \rho\left[\left(\operatorname{div}_{\Gamma}\left(\mathcal{R}-\frac{1}{2} \mathcal{H}\right) \nabla_{\Gamma}\right)-\frac{1}{2} \mathcal{H} \kappa_{i}^{2}\right] r_{[2]}^{\varepsilon}=O\left(\varepsilon^{3}\right) \text { on } \Gamma
$$

where $O(x)$ stands for a generic distribution belonging to $H^{-\frac{3}{2}}(\Gamma)$.

Proof. We have on $\Gamma$

$$
\partial_{\boldsymbol{n}} r_{[2]}^{\varepsilon}+\rho \varepsilon\left(\operatorname{div}_{\Gamma} \nabla_{\Gamma}+\kappa_{i}^{2}\right) r_{[2]}^{\varepsilon}+\rho \varepsilon^{2}\left[\left(\operatorname{div}_{\Gamma}\left(\mathcal{R}-\frac{1}{2} \mathcal{H}\right) \nabla_{\Gamma}\right)-\frac{1}{2} \mathcal{H} \kappa_{i}^{2}\right] r_{[2]}^{\varepsilon}=F_{[2]}^{\varepsilon, 2}-F_{[2]}^{\varepsilon, 1}=R_{1}^{\varepsilon}+R_{2}^{\varepsilon}
$$


where

$$
\begin{aligned}
R_{1}^{\varepsilon}= & \varepsilon^{2} \frac{\rho}{2} \operatorname{div}_{\Gamma}\left[\left(2 D_{\Gamma}^{2}(\boldsymbol{\theta} \cdot \boldsymbol{n})-\Delta_{\Gamma}(\boldsymbol{\theta} \cdot \boldsymbol{n}) \mathrm{I}\right) \nabla_{\Gamma}\left(v_{[2]}^{\varepsilon}+u^{i n c}\right)\right]-\varepsilon^{2} \frac{\rho}{2} \kappa_{i}^{2}\left[\Delta_{\Gamma}(\boldsymbol{\theta} \cdot \boldsymbol{n})\left(v_{[2]}^{\varepsilon}+u^{i n c}\right)\right] \\
& -\frac{\rho}{2} \varepsilon^{2}\left(\Delta_{\Gamma}+\kappa_{i}^{2}\right)\left[\operatorname{div}_{\Gamma}(\boldsymbol{\theta} \cdot \boldsymbol{n}) \nabla_{\Gamma}+(\boldsymbol{\theta} \cdot \boldsymbol{n}) \kappa_{i}^{2}\right]\left(v_{[2]}^{\varepsilon}+u^{i n c}\right) \\
& -\frac{\rho}{2} \varepsilon^{2}\left[\operatorname{div}_{\Gamma}(\boldsymbol{\theta} \cdot \boldsymbol{n}) \nabla_{\Gamma}+(\boldsymbol{\theta} \cdot \boldsymbol{n}) \kappa_{i}^{2}\right]\left(\Delta_{\Gamma}+\kappa_{i}^{2}\right)\left(v_{[2]}^{\varepsilon}+u^{i n c}\right)
\end{aligned}
$$

and where

$$
\begin{aligned}
R_{2}^{\varepsilon}= & -\varepsilon^{2} \rho \operatorname{div}_{\Gamma}\left[(\boldsymbol{\theta} \cdot \boldsymbol{n})\left(\mathcal{R}-\frac{1}{2} \mathcal{H} I\right) \nabla_{\Gamma}-\frac{1}{2} \kappa_{i}^{2}(\boldsymbol{\theta} \cdot \boldsymbol{n}) \mathcal{H}\right] \partial_{\boldsymbol{n}}\left(v_{[2]}^{\varepsilon}+u^{i n c}\right) \\
& -\varepsilon \rho\left[\Delta_{\Gamma}+\kappa_{i}^{2}\right]\left((\boldsymbol{\theta} \cdot \boldsymbol{n}) \partial_{\boldsymbol{n}}\left(v_{[2]}^{\varepsilon}+u^{i n c}\right)\right) \\
& +\varepsilon(\rho-1)\left[\operatorname{div}_{\Gamma}\left(1+\varepsilon\left(\mathcal{R}-\frac{1}{2} \mathcal{H}\right)\right) \nabla_{\Gamma}+\left(1-\frac{1}{2} \varepsilon \mathcal{H}\right) \kappa_{i}^{2}\right]\left((\boldsymbol{\theta} \cdot \boldsymbol{n}) \partial_{\boldsymbol{n}}\left(v_{[2]}^{\varepsilon}+u^{i n c}\right)\right) \\
= & \left.-\varepsilon^{2} \rho \operatorname{div}_{\Gamma}\left[(\boldsymbol{\theta} \cdot \boldsymbol{n})\left(\mathcal{R}-\frac{1}{2} \mathcal{H} I\right) \nabla_{\Gamma}-\frac{1}{2} \kappa_{i}^{2}(\boldsymbol{\theta} \cdot \boldsymbol{n}) \mathcal{H}\right] \partial_{\boldsymbol{n}}\left(v_{[2]}^{\varepsilon}+u^{i n c}\right)\right) \\
& -\varepsilon \rho\left[\Delta_{\Gamma}+\kappa_{i}^{2}\right]\left((\boldsymbol{\theta} \cdot \boldsymbol{n}) \partial_{\boldsymbol{n}}\left(v_{[2]}^{\varepsilon}+u^{i n c}\right)\right)+\varepsilon(\rho-1)\left[\Delta_{\Gamma}+\kappa_{i}^{2}\right]\left((\boldsymbol{\theta} \cdot \boldsymbol{n}) \partial_{\boldsymbol{n}}\left(v_{[2]}^{\varepsilon}+u^{i n c}\right)\right) \\
& +\varepsilon^{2}(\rho-1)\left[\operatorname{div}_{\Gamma}\left(\mathcal{R}-\frac{1}{2} \mathcal{H}\right) \nabla_{\Gamma}-\frac{1}{2} \mathcal{H} \kappa_{i}^{2}\right]\left((\boldsymbol{\theta} \cdot \boldsymbol{n}) \partial_{\boldsymbol{n}}\left(v_{[2]}^{\varepsilon}+u^{i n c}\right)\right) .
\end{aligned}
$$

Thanks to the relation

$$
\partial_{\boldsymbol{n}}\left(v_{[2]}^{\varepsilon}+u^{i n c}\right)=-B^{\varepsilon, 2}\left(v_{[2]}^{\varepsilon}+u^{i n c}\right)
$$

where

$$
B^{\varepsilon, 2}=\varepsilon \rho\left[\Delta_{\Gamma}+\kappa_{i}^{2}\right]+\varepsilon^{2} \rho\left[\operatorname{div}_{\Gamma}\left(\mathcal{R}-\frac{1}{2} \mathcal{H}\right) \nabla_{\Gamma}-\frac{1}{2} \mathcal{H} \kappa_{i}^{2}\right]
$$

we can rewrite $R_{2}^{\varepsilon}$ as

$$
R_{2}^{\varepsilon}=\varepsilon^{2} \rho\left[\Delta_{\Gamma}+\kappa_{i}^{2}\right]\left((\boldsymbol{\theta} \cdot \boldsymbol{n})\left(\Delta_{\Gamma}+\kappa_{i}^{2}\right)\left(v_{[2]}^{\varepsilon}+u^{i n c}\right)\right)+O\left(\varepsilon^{3}\right)
$$

where $O(x)$ stands for a generic distribution belonging to $H^{-\frac{3}{2}}(\Gamma)$. Thanks to the Proposition 6.2 , we conclude

$$
\begin{aligned}
F_{[2]}^{\varepsilon, 2}-F_{[2]}^{\varepsilon, 1}= & \varepsilon^{2} \frac{\rho}{2} \operatorname{div}_{\Gamma}\left[\left(2 D_{\Gamma}^{2}(\boldsymbol{\theta} \cdot \boldsymbol{n})-\Delta_{\Gamma} \boldsymbol{\theta} \cdot \boldsymbol{n} I\right) \nabla_{\Gamma}\left(v_{[2]}^{\varepsilon}+u^{i n c}\right)\right]-\varepsilon^{2} \frac{\rho}{2} \kappa_{i}^{2}\left[\Delta_{\Gamma}(\boldsymbol{\theta} \cdot \boldsymbol{n})\left(v_{[2]}^{\varepsilon}+u^{i n c}\right)\right] \\
& -\frac{\rho}{2} \varepsilon^{2}\left(\Delta_{\Gamma}+\kappa_{i}^{2}\right)\left[\operatorname{div}_{\Gamma}(\boldsymbol{\theta} \cdot \boldsymbol{n}) \nabla_{\Gamma}+(\boldsymbol{\theta} \cdot \boldsymbol{n}) \kappa_{i}^{2}\right]\left(v_{[2]}^{\varepsilon}+u^{i n c}\right) \\
& -\frac{\rho}{2} \varepsilon^{2}\left[\operatorname{div}_{\Gamma}(\boldsymbol{\theta} \cdot \boldsymbol{n}) \nabla_{\Gamma}+(\boldsymbol{\theta} \cdot \boldsymbol{n}) \kappa_{i}^{2}\right]\left(\Delta_{\Gamma}+\kappa_{i}^{2}\right)\left(v_{[2]}^{\varepsilon}+u^{i n c}\right) \\
& +\varepsilon^{2} \rho\left[\Delta_{\Gamma}+\kappa_{i}^{2}\right]\left(\boldsymbol{\theta} \cdot \boldsymbol{n}\left(\Delta_{\Gamma}+\kappa_{i}^{2}\right)\left(v_{[2]}^{\varepsilon}+u^{i n c}\right)\right)+O\left(\varepsilon^{3}\right) \\
= & O\left(\varepsilon^{3}\right) .
\end{aligned}
$$

Proof of Theorem 6.1. We can easily verify that $r_{[N]}^{\varepsilon}$ solves the following boundary value problem:

$$
\left\{\begin{array}{cl}
\Delta r_{[N]}^{\varepsilon}+\kappa_{e}^{2} r_{[N]}^{\varepsilon} & =0 \text { in } \Omega_{e x t} \\
\lim _{R \rightarrow+\infty} \int_{\partial B_{R}}\left|\frac{\partial r_{[N]}^{\varepsilon}}{\partial r}-i k_{e} r_{[N]}^{\varepsilon}\right|^{2} d s & =0
\end{array}\right.
$$

with the GIBC on $\Gamma$

$$
\partial_{n} r_{[2]}^{\varepsilon}+\rho \varepsilon\left(\operatorname{div}_{\Gamma}\left[\left(I+\varepsilon\left(\mathcal{R}-\frac{1}{2} \mathcal{H} \mathrm{I}\right)\right) \nabla_{\Gamma} r_{[2]}^{\varepsilon}\right]\right)+\rho \kappa_{i}^{2} \varepsilon\left(1-\frac{\varepsilon}{2} \mathcal{H}\right) r_{[2]}^{\varepsilon} .=O\left(\varepsilon^{3}\right) \text { on } \Gamma
$$

We follows ideas of [32, Lemma 1]. We begin to introduce the Dirichlet to Neumann operator $\mathcal{A}_{\Gamma}$ : $H^{s+\frac{1}{2}}(\Gamma) \mapsto H^{s-\frac{1}{2}}(\Gamma), \quad s \in \mathbb{R}$ defined as follows : for $g \in H^{s+\frac{1}{2}}(\Gamma)$ we set $\mathcal{A}_{\Gamma}(g)=\partial_{\boldsymbol{n}} v$ the normal derivative of $v$ solution of

$$
\begin{aligned}
& \left\{\begin{array}{rlrl}
\Delta v+\kappa_{e}^{2} v & & =0, \text { in } \Omega_{\text {ext }} \\
v & & =g, \text { on } \Gamma \\
\lim _{R \rightarrow+\infty} \int_{\partial B_{R}}\left|\frac{\partial r_{[N]}^{\varepsilon}}{\partial r}-i k_{e} r_{[N]}^{\varepsilon}\right|^{2} d r & =0 .
\end{array}\right. \\
& \left\|\mathcal{A}_{\Gamma} u\right\|_{L^{2}(\Gamma)} \leq c_{3}\|u\|_{H^{1}}
\end{aligned}
$$

Let $\mathcal{Z}_{\Gamma}$ be the operator defined by

$$
\mathcal{Z}_{\Gamma} w=\rho \varepsilon\left(\operatorname{div}_{\Gamma}\left[\left(I+\varepsilon\left(\mathcal{R}-\frac{1}{2} \mathcal{H} \mathrm{I}\right)\right) \nabla_{\Gamma} w\right]\right)+\rho \kappa_{i}^{2} \varepsilon\left(1-\frac{\varepsilon}{2} \mathcal{H}\right) w
$$


Set

$$
\mathcal{B}_{\Gamma}=\mathcal{A}_{\Gamma}+\mathcal{Z}_{\Gamma} .
$$

We study first $\mathcal{B}_{\Gamma}^{\Lambda}=\mathcal{A}_{\Gamma}+\rho \varepsilon\left(\operatorname{div}_{\Gamma}\left[\left(I+\varepsilon\left(\mathcal{R}-\frac{1}{2} \mathcal{H} \mathrm{I}\right)\right) \nabla_{\Gamma} w\right]\right)-\Lambda u$ where $\Lambda$ is a positive constant chosen sufficiently large. Denote $E=\rho \varepsilon\left(1-\varepsilon \frac{H}{2}\right) I+\rho \varepsilon^{2} \mathcal{R}$. Supposing $\varepsilon>0$ sufficiently small such that the spectrum of the matrix $E$ belongs to $\left(c_{1}, c_{2}\right)$ for some $c_{1}, c_{2}>0$. We have for $u \in H^{1}(\Gamma)$

$$
\begin{aligned}
-\operatorname{Re}\left(\left\langle\mathcal{B}_{\Gamma}^{\Lambda} u, u\right\rangle\right) & =-\left\langle\mathcal{A}_{\Gamma} u, u\right\rangle+\left\langle\nabla_{\Gamma} u, E \nabla_{\Gamma} u\right\rangle+\Lambda\|u\|_{L^{2}(\Gamma)}^{2} \\
& \geq-\left\|\mathcal{A}_{\Gamma} u\right\|_{L^{2}(\Gamma)}\|u\|_{L^{2}(\Gamma)}+c_{1}\|u\|_{H^{1}(\Gamma)}^{2}+\left(c_{1}+\Lambda\right)\|u\|_{L^{2}}^{2} \\
& \geq-c_{3}\|u\|_{H^{1}(\Gamma)}\|u\|_{L^{2}(\Gamma)}+c_{1}\|u\|_{H^{1}(\Gamma)}^{2}+\left(c_{1}+\Lambda\right)\|u\|_{L^{2}}^{2} \\
& \geq-c_{3}\left(\frac{\delta}{2}\|u\|_{H^{1}(\Gamma)}^{2}+\frac{\|u\|_{L^{2}(\Gamma)}^{2}}{2 \delta}+c_{1}\|u\|_{H^{1}(\Gamma)}^{2}+\left(c_{1}+\Lambda\right)\|u\|_{L^{2}}^{2}\right. \\
& \left.\geq\left(c_{1}-\frac{c_{3}}{2 \delta}\right)\|u\|_{H^{1}(\Gamma)}^{2}+\left(c_{1}+\Lambda-\frac{c_{3}}{2 \delta}\right)\|u\|_{L^{2}(\Gamma)}^{2}\right)
\end{aligned}
$$

The last two inequalities are obtained thanks to the Young's inequality. Setting $\delta=\frac{c_{1}}{c_{3}}$ we then get

$$
-\operatorname{Re}\left(\left\langle\mathcal{B}_{\Gamma}^{\Lambda}\langle u, u\rangle\right) \geq \frac{c_{1}}{2}\|u\|_{H^{1}(\Gamma)}^{2}+\left(\Lambda+c_{1}-\frac{c_{3}^{2}}{2 c_{1}}\right)\|u\|_{L^{2}(\Gamma)}^{2} .\right.
$$

Hence coercivity is obtained whenever $\Lambda \geq \frac{c_{3}^{2}}{2 c_{1}}-c_{1}$. We have shown that $-\mathcal{B}_{\Gamma}=-\mathcal{B}_{\Gamma}^{\Lambda}+K_{\Gamma}$ where $-K_{\Gamma}=\Lambda u$ is compact. Then $\mathcal{B}_{\lambda}$ is a Fredholm operator of index zero. Furthermore, one can easily show that $\mathcal{B}_{\Gamma}$ is injective [12. The trick is classical : we combine the Reillich theorem and the analytic continuation principle. Then it is invertible. Since $\mathcal{B}_{\Gamma}$ is bounded from $H^{1}(\Gamma)$ to $H^{-1}(\Gamma)$, we deduce from the closed graph theorem, that if $-B_{\Gamma} u=h$ then there exists $C>0$ such that

$$
\|u\|_{H^{1}(\Gamma)} \leq C\|h\|_{H^{-1}(\Gamma)} .
$$

Doing the same analysis for $h \in H^{s}(\Gamma)$ when $s>-1$, we deduce that the problem has a unique solution $u \in H^{1}(\Gamma)$. Hence $-\mathcal{B}_{\Gamma}^{\Lambda} u+u=h+\mathcal{A}_{\Gamma} u+(1-\Lambda) u \in H^{\min (s, 0)}(\Gamma)$ and since $\Gamma$ is $C^{\infty}$ we get $u \in H^{s+2}(\Gamma)$ thanks to bootstrap procedure. We conclude from the closed graph theorem that there exists $C>0$ such that

$$
\|u\|_{H^{s+2}(\Gamma)} \leq C\|h\|_{H^{s}(\Gamma)} .
$$

The case $s<-1$ is treated by transposition. Indeed, for any $s \in \mathbb{R}$ the operator $\mathcal{B}_{\Gamma}$ continuously maps $H^{s+2}(\Gamma)$ to $H^{s}(\Gamma)$. Its $L^{2}$-adjoint is $\mathcal{B}_{\Gamma}: H^{-s}(\Gamma) \rightarrow H^{-s-2}(\Gamma)$, which is bijective as $-s-2>-1$ by previous case. The proof is then completed as in the previous case. If $s=-3 / 2$ we get the first estimate. We obtain the other estimates by the potential theory.

Similar bounds can be obtained in the Dirichlet case. Indeed, we can prove again that $\left(F_{[N \mid}^{\varepsilon, 1}-F_{\mid N]}^{\varepsilon, 2}\right)$ is up to $O\left(\varepsilon^{N+1}\right)$ in the following lemma.

Lemma 6.4. In the Dirichlet case, we have

$$
r_{[2]}^{\varepsilon}-\frac{1}{\rho} \varepsilon\left(1+\frac{\varepsilon}{2} \mathcal{H}\right) \partial_{n} r_{[2]}^{\varepsilon}=O\left(\varepsilon^{3}\right) \text { on } \Gamma
$$

where $O(x)$ stands for a generic distribution belonging to $H^{-\frac{1}{2}}(\Gamma)$.

Proof. We have on $\Gamma$

$$
r_{[2]}^{\varepsilon}-\frac{1}{\rho} \varepsilon\left(1+\frac{\varepsilon}{2} \mathcal{H}\right) \partial_{n} r_{[2]}^{\varepsilon}=F_{[2]}^{\varepsilon, 2}-F_{[2]}^{\varepsilon, 1}=R_{1}^{\varepsilon}+R_{2}^{\varepsilon},
$$

where

$$
\begin{gathered}
R_{1}^{\varepsilon}=\varepsilon\left(1+\frac{1}{2} \varepsilon \mathcal{H}\right)\left[\operatorname{div}_{\Gamma}(\boldsymbol{\theta} \cdot \boldsymbol{n}) \nabla_{\Gamma}+(\boldsymbol{\theta} \cdot \boldsymbol{n}) \kappa_{i}^{2}\right]\left(v_{[2]}^{\varepsilon}+u^{i n c}\right), \\
R_{2}^{\varepsilon}=-\frac{1}{2 \rho} \varepsilon^{2}\left[\Delta_{\Gamma}+\kappa_{i}^{2}\right]\left((\boldsymbol{\theta} \cdot \boldsymbol{n}) \partial_{\boldsymbol{n}}\left(v_{[2]}^{\varepsilon}+u^{i n c}\right)\right)-\frac{1}{2 \rho} \varepsilon^{2}(\boldsymbol{\theta} \cdot \boldsymbol{n})\left[\Delta_{\Gamma}+\kappa_{i}^{2}\right] \partial_{\boldsymbol{n}}\left(v_{[2]}^{\varepsilon}+u^{i n c}\right) \\
+\frac{1}{2 \rho} \varepsilon^{2}\left(\Delta_{\Gamma}(\boldsymbol{\theta} \cdot \boldsymbol{n})\right) \partial_{\boldsymbol{n}}\left(v_{[2]}^{\varepsilon}+u^{i n c}\right) .
\end{gathered}
$$

Thanks to the relation

$$
\left(v_{[2]}^{\varepsilon}+u^{i n c}\right)=-B^{\varepsilon, 2} \partial_{\boldsymbol{n}}\left(v_{[2]}^{\varepsilon}+u^{i n c}\right),
$$


where

we can rewrite $R_{1}^{\varepsilon}$ as

$$
B^{\varepsilon, 2}=-\frac{1}{\rho} \varepsilon \mathrm{I}-\frac{1}{1 \rho} \varepsilon^{2} \mathcal{H} \mathrm{I}
$$

$$
R_{1}^{\varepsilon}=\varepsilon^{2} \frac{1}{\rho}\left[\operatorname{div}_{\Gamma}(\boldsymbol{\theta} \cdot \boldsymbol{n}) \nabla_{\Gamma}+(\boldsymbol{\theta} \cdot \boldsymbol{n}) \kappa_{i}^{2}\right] \partial_{\boldsymbol{n}}\left(v_{[2]}^{\varepsilon}+u^{i n c}\right)+O\left(\varepsilon^{3}\right),
$$

where $O(x)$ stands for a generic distribution belonging to $H^{-\frac{1}{2}}(\Gamma)$. We also simplify the expression of $R_{2}^{\varepsilon}$ as

$$
R_{2}^{\varepsilon}=-\frac{1}{\rho} \varepsilon^{2} \nabla_{\Gamma}(\boldsymbol{\theta} \cdot \boldsymbol{n}) \cdot \nabla_{\Gamma} \partial_{\boldsymbol{n}}\left(v_{[2]}^{\varepsilon}+u^{i n c}\right)-\frac{1}{\rho} \varepsilon^{2}(\boldsymbol{\theta} \cdot \boldsymbol{n})\left[\Delta_{\Gamma}+\kappa_{i}^{2}\right] \partial_{\boldsymbol{n}}\left(v_{[2]}^{\varepsilon}+u^{i n c}\right) .
$$

We finally get $F_{[2]}^{\varepsilon, 2}-F_{[2]}^{\varepsilon, 1}=O\left(\varepsilon^{3}\right)$.

\section{Numerical experiments}

We discuss in this section the numerical accuracy of the GIBCs to approximate the solution to the original transmission problem and its shape derivatives. We evaluate the $L^{2}$ error of the far-field pattern associated to the exact and approximate fields. The far-field pattern are computed by solving the boundary integral equations given in the Appendix thanks to the high-order spectral algorithm presented in [18. The numerical scheme applies to boundaries which are globally parameterised by spherical coordinates and consists in transforming the integral equation on the surface $\Gamma$ to an integral equation on the unit sphere using a change of variable and then by expanding the integrand and looking for a scalar solution in terms of series of scalar spherical harmonics.

We denote by $\left(Y_{\ell}^{j}\right)_{0 \leq|j| \leq \ell}$ the orthonormal system of scalar spherical harmonics. In all our experiments we set $\kappa_{e}=\pi$ so that the object size is roughly $1 \lambda_{e}$ (where $\lambda_{e}=\frac{2 \pi}{\kappa_{e}}$ is the exterior wavelenght) and $\kappa_{i}=2 \kappa_{e}$, $\rho=1.3$. The incident plane wave is defined by $u^{i n c}(\boldsymbol{x})=e^{i \kappa_{e} \boldsymbol{x} \cdot \boldsymbol{d}}$, with $\boldsymbol{d}={ }^{\top}(1,0,0)$.

As a first test, we consider a spherical coating of an acoustic object whose boundary $\Gamma_{1}^{\varepsilon}$ is parameterised by the unit sphere $\mathbb{S}^{2}$ as follows and the visualisation is given in Figure 1

$$
\boldsymbol{x} \in \Gamma_{1}^{\varepsilon} \Leftrightarrow \exists \hat{\boldsymbol{x}} \in \mathbb{S}^{2}, \boldsymbol{x}=r_{1}(\hat{\boldsymbol{x}}) \hat{\boldsymbol{x}} \text { with } r_{1}=0.9 \sqrt{4 \pi} Y_{0}^{0}+0.1 Y_{10}^{5}+0.1 Y_{10}^{-5} .
$$

The width is then $\varepsilon h=1-r_{1}$. In Table 1 we indicate the $L^{2}$ relative error, denoted by $\operatorname{err}_{2}$ between the

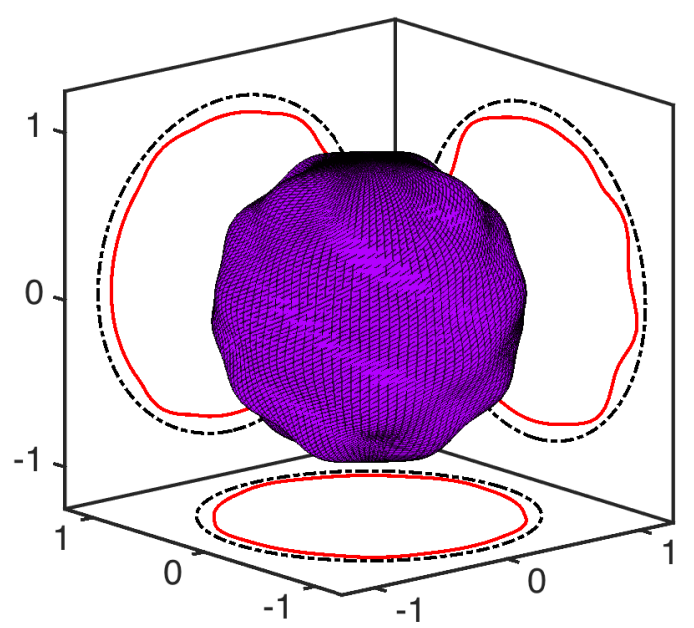

Figure 1: Interior boundary of the spherical coating with a variable thickness $\varepsilon h$ varying between $0.05 \lambda_{e}$ and $0.15 \lambda_{e}$.

far-field patterns of the exact exterior field $u_{e x t}^{\varepsilon}$ and the approximated field $v_{[N \mid}^{\varepsilon}$.

$$
\operatorname{err}_{2}^{\varepsilon}=\left(\frac{\int_{\mathbb{S}^{2}}\left|v_{\infty,[N]}^{\varepsilon}-u_{\infty}^{\varepsilon}\right|^{2} d s}{\int_{\mathbb{S}^{2}}\left|u_{\infty}^{\varepsilon}\right|^{2} d s}\right)^{\frac{1}{2}}
$$


In Figures 2 and 3 we compare the amplitude of the far-field patterns of the exact solution and the approximate solution on the cut $\phi=0^{\circ}$.

Table 1: Scattering of an incident plane wave by the spherical coating with either a Dirichlet or a Neumann boundary condition on the interior boundary : relative $L^{2}$-errors between the true scattered field and the approximate field.

\begin{tabular}{cc||c}
\hline \hline & Dirichlet & Neumann \\
\cline { 2 - 3 }$N$ & err $_{2}^{\varepsilon}$ & err $_{2}^{\varepsilon}$ \\
\hline 0 & $2.9132 \mathrm{E}-01$ & $1.1004 \mathrm{E}-00$ \\
1 & $5.0567 \mathrm{E}-02$ & $2.1414 \mathrm{E}-01$ \\
2 & $2.9561 \mathrm{E}-02$ & $1.3574 \mathrm{E}-01$ \\
3 & $7.5691 \mathrm{E}-03$ & $4.5420 \mathrm{E}-02$ \\
\hline
\end{tabular}
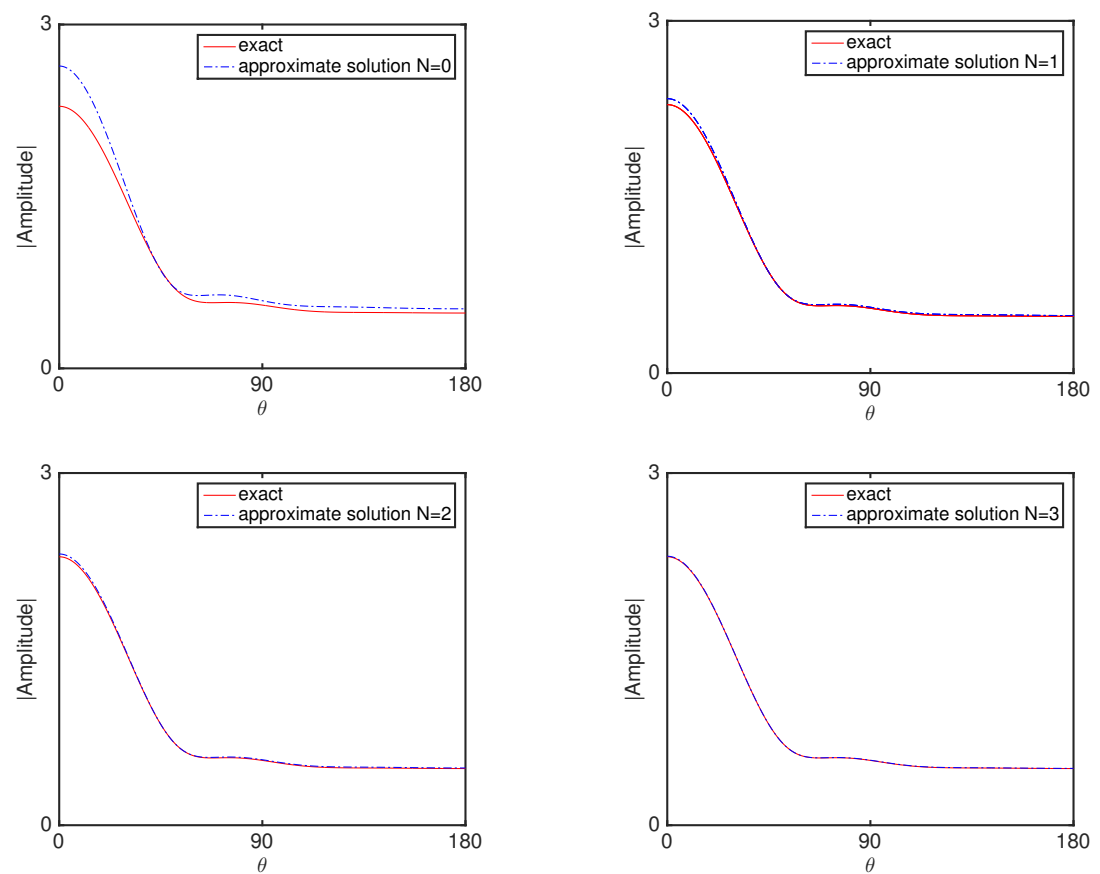

Figure 2: Amplitude of the farfield patterns $u_{\infty}^{\varepsilon}$ and $v_{\infty,[N]}^{\varepsilon}$ of the scattered field associated to the original transmission problems for the spherical coating and its approximate fields in the case of a Dirichlet interior boundary condition.

As a second task, we consider an axisymetrical coating with a constant thickness whose the exterior boundary $\Gamma_{2}$ is parameterised by the unit sphere as follows and the visualisation is given in Figure 4.

$$
\boldsymbol{x} \in \Gamma_{2} \Leftrightarrow \exists \hat{\boldsymbol{x}} \in \mathbb{S}^{2}, \boldsymbol{x}=r_{2}(\hat{\boldsymbol{x}}) \hat{\boldsymbol{x}} \text { with } r_{2}=\frac{5}{6} \sqrt{4 \pi} Y_{0}^{0}+\frac{1}{4} Y_{1}^{0}+\frac{1}{4} Y_{2}^{0} .
$$

The curvature operator $\mathcal{R}$, the mean curvature $\mathcal{H}$ and the gauss curvature $\mathcal{G}$ are computed analytically. The surface differential operators are computed using integration by parts, projection onto the spherical harmonics and the various formulas stated in 23 .

To attest the shape derivative formulas of $u_{e x t}^{\varepsilon}$ and its approximate field $v_{[N]}^{\varepsilon}$ given by Theorems 4.1, 5.1 and 5.2 , we compare them with the Gâteaux derivative typically defined by

$$
\dot{u}_{\infty}^{\varepsilon}=\lim _{t \rightarrow 0}\left(\dot{u}_{\infty}^{\varepsilon, t}=\frac{u_{\infty}^{\varepsilon, t \boldsymbol{\theta}}-u_{\infty}^{\varepsilon}}{t}\right) \quad \text { and } \quad \dot{v}_{\infty,[N]}^{\varepsilon}=\lim _{t \rightarrow 0}\left(\dot{v}_{\infty,[N]}^{\varepsilon, t}=\frac{v_{\infty,[N]}^{\varepsilon, t \boldsymbol{\theta}}-v_{\infty,[N]}^{\varepsilon}}{t}\right),
$$

for different values of $t$ and $N=2$. The direction $\boldsymbol{\theta}$ is described by the spherical coordinates of any point $\hat{\boldsymbol{x}} \in \mathbb{S}^{2}$ denoted by $(\psi, \phi) \in(0 ; \pi) \times(0 ; 2 \pi) \cup\{(0,0) ;(0, \pi)\}$ by

$$
\boldsymbol{\theta}(\hat{\boldsymbol{x}})=r(\hat{\boldsymbol{x}}) \hat{\boldsymbol{x}}, \quad r(\hat{\boldsymbol{x}})=\frac{3}{5} \cos \psi \sin \phi+\frac{2}{5} \cos 2 \psi \sin 3 \phi .
$$



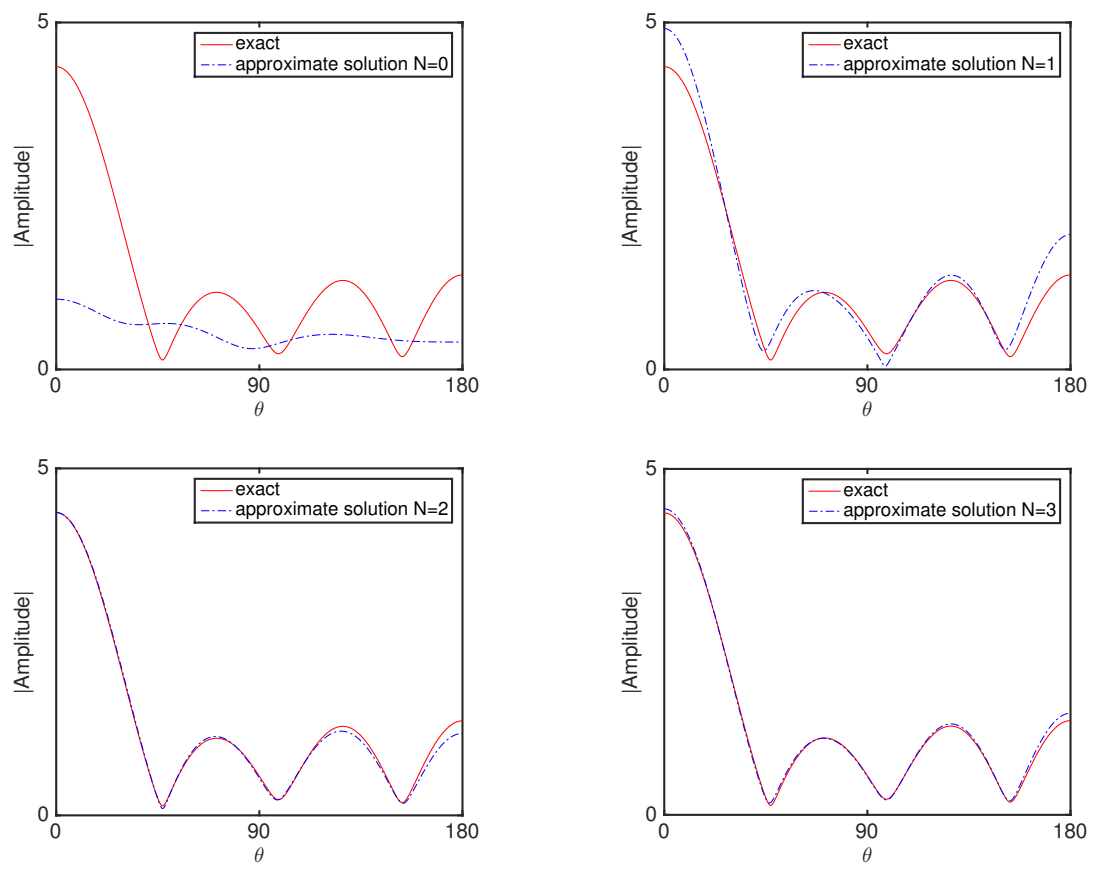

Figure 3: Amplitude of the farfield patterns $u_{\infty}^{\varepsilon}$ and $v_{\infty,[N]}^{\varepsilon}$ of the scattered field associated to the original transmission problems for the spherical coating and its approximate fields in the case of a Neumann interior boundary condition.

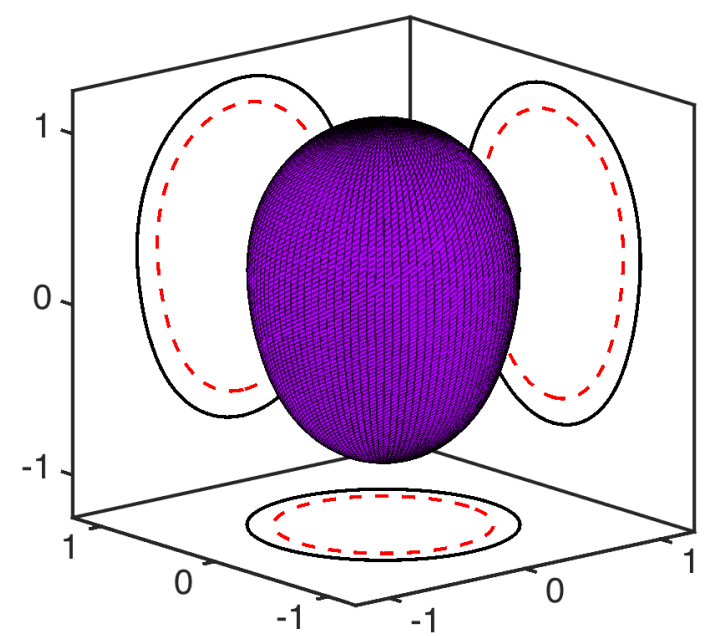

Figure 4: Exterior boundary of a coated object with a constant thickness $\varepsilon=0.15 \lambda_{e}$.

We set $\varepsilon=0.1 \lambda_{e}$. As expected, we observe linear convergence rate.

In Figure 5, we numerically verify the accuracy and effectiveness of the two approaches presented in Section 4 and 5 to compute of the shape derivatives. The curves represents the $L^{2}$ error between the shape derivative of the true far-field $\dot{u}_{\infty}^{\varepsilon}$ and the approximate shape derivatives $w_{\infty,[N]}^{\varepsilon}$ or $\dot{v}_{\infty,[N]}^{\varepsilon}$ for $N=1,2$ and various values on the thickness $\varepsilon$ in a $\log \log$ scale. In view of the error bound given in the introduction and the Theorem 6.1 taking the logarithm to both sides we obtain

$$
\log _{10}\left(\left\|u_{\infty}^{\varepsilon}-v_{\infty,[N]}^{\varepsilon}\right\|_{L^{2}}\right) \approx \log _{10}\left(c_{N}\right)+m_{N} \log _{10}(\varepsilon)
$$

where $c_{N}$ is a constant and $m_{N} \approx N+1$. The positive real values $m_{N}$ are the slope of the linear curves and 
Table 2: Numerical computation of the Fréchet derivative : comparison with the finite difference method

\begin{tabular}{ccc||cc}
\hline \hline & \multicolumn{2}{c||}{ Dirichlet } & \multicolumn{1}{c}{ Neumann } \\
\cline { 2 - 3 }$t$ & $\frac{\left\|\dot{u}_{\infty}^{\varepsilon, t}-\dot{u}_{\infty}^{\varepsilon}\right\|_{L^{2}}}{\left\|\dot{u}_{\infty}^{\varepsilon}\right\|_{L^{2}}}$ & $\frac{\left\|\dot{v}_{\infty,[2]}^{\varepsilon, t}-\dot{v}_{\infty,[2]}^{\varepsilon}\right\|_{L^{2}}}{\left\|\dot{v}_{\infty,[2]}^{\varepsilon}\right\|_{L^{2}}}$ & $\frac{\left\|\dot{u}_{\infty}^{\varepsilon}-\dot{u}_{\infty}^{\varepsilon}\right\|_{L^{2}}}{\left\|\dot{u}_{\infty}^{\varepsilon}\right\|_{L^{2}}}$ & $\frac{\left\|\dot{v}_{\infty,[2]}^{\varepsilon, t}-\dot{v}_{\infty,[2]}^{\varepsilon}\right\|_{L^{2}}}{\left\|\dot{v}_{\infty,[2]}^{\varepsilon}\right\|_{L^{2}}}$ \\
\hline $\mathrm{E}-01$ & $1.1892 \mathrm{E}-01$ & $1.2944 \mathrm{E}-01$ & $2.8351 \mathrm{E}-01$ & $1.1807 \mathrm{E}-01$ \\
$\mathrm{E}-02$ & $1.1899 \mathrm{E}-02$ & $1.2969 \mathrm{E}-02$ & $2.7977 \mathrm{E}-02$ & $1.1832 \mathrm{E}-02$ \\
$\mathrm{E}-03$ & $1.1899 \mathrm{E}-03$ & $1.2970 \mathrm{E}-03$ & $2.7975 \mathrm{E}-03$ & $1.1832 \mathrm{E}-03$ \\
\hline
\end{tabular}

are indicated on the Figure 5. We observe that similar speed of convergence of $v_{\infty,[N]}^{\varepsilon}$ to $u_{\infty}^{\varepsilon}$ and of $\dot{v}_{\infty,[N]}^{\varepsilon}$ to $\dot{u}_{\infty}^{\varepsilon}$ are obtained. The speed of convergence of $w_{\infty,[N]}^{\varepsilon}$ to $\dot{u}_{\infty}^{\varepsilon}$ is close to $N+1$ but slower than the one of $\dot{v}_{\infty,[N]}^{\varepsilon}$ to $\dot{u}_{\infty}^{\varepsilon}$.

\section{Conclusion and perspectives}

In this work we proposed a new way to construct GIBCs for elliptic problems in $\mathbb{R}^{3}$ extending previous work realised in $\mathbb{R}^{2}$ [33, 3]. The method allows the possibility to obtain, in future works, the high order GIBCs modelling thin coatings in electromagnetics and elastodynamics with variable thickness, generalising the already existing results [2, 5, 6, 10, 17, 19].

Using these results, we investigate the asymptotic behaviour of the shape derivatives of the solution to thin layer transmission problems. We present two different way to approach the shape derivatives. On one hand, we construct the GIBCs associated to the thin layer transmission problem characterising the shape derivative of the solution. On the other hand, we compute the shape derivatives of the approximate solution. We show that the two approaches are equivalent in the sense that the error estimates is up to $O\left(\varepsilon^{N+1}\right)$, where $N$ is the order of truncation in the asymptotic expansion of the exact shape derivative. We explain the results by the fact that the first shape derivative depends only on the normal deformation of the exterior boundary. A general observation is that for $N \geq 2$, the first approach is simpler than computing the shape derivatives of the GIBCs.

\section{Appendix: boundary integral equation methods}

In this appendix we present the boundary integral equation methods used to solve both the thin layer transmission problem and the boundary value problems with GIBCs. We follow the procedure described in 34] for the transmission problem and in [18 for impedance-like problems. For more details on the potential theory in acoustics we refer to [13.

For $a=i, e$, let $G\left(\kappa_{a}, \boldsymbol{z}\right)=\frac{e^{i \kappa_{a}|\boldsymbol{z}|}}{4 \pi|\boldsymbol{z}|}$ be the fundamental solution of the Helmholtz equation $\Delta u+\kappa_{a}^{2} u=0$.

We denote by $\mathcal{S}_{\Gamma}^{a}$ and $\mathcal{D}_{\Gamma}^{a}$ the single layer and double layer potential operators related to the boundary $\Gamma$. They are defined by

$$
\mathcal{S}_{\Gamma}^{a} \varphi(\boldsymbol{x})=\int_{\Gamma} G\left(\kappa_{a}, \boldsymbol{x}-\boldsymbol{y}\right) \varphi(\boldsymbol{y}) d \sigma(\boldsymbol{y}) \text { and } \mathcal{D}_{\Gamma}^{a} \psi(\boldsymbol{x})=\int_{\Gamma} \partial_{\boldsymbol{n}}^{\boldsymbol{y}} G\left(\kappa_{a}, \boldsymbol{x}-\boldsymbol{y}\right) \psi(\boldsymbol{y}) d \sigma(\boldsymbol{y}) .
$$

The functions $\varphi$ and $\psi$ are some distributions in the Sobolev spaces of fractional order $H^{\frac{1}{2}}(\Gamma)$ and $H^{-\frac{1}{2}}(\Gamma)$, respectively. We define in the same way the potential operators related to the boundary $\Gamma^{\varepsilon}$. The transmission problem 1.1 can be reduced in several different ways to a system of uniquely solvable boundary integral equations. We present two different approaches. The indirect approach is based on the layer ansatz

$$
\begin{gathered}
u_{\text {ext }}^{\varepsilon}=\rho \mathcal{D}_{\Gamma}^{e} \psi-\mathcal{S}_{\Gamma^{e}}^{e}, \quad \text { in } \Omega_{e x t}, \\
u_{i n t}^{\varepsilon}=\left(\mathcal{D}_{\Gamma}^{i} \psi-\mathcal{S}_{\Gamma^{i}}^{i} \varphi\right)+\left(\mathcal{D}_{\Gamma^{\varepsilon}}^{i} \xi^{\varepsilon}+i \eta \mathcal{S}_{\Gamma^{\varepsilon}}^{i} \xi^{\varepsilon}\right), \quad \text { in } \Omega_{i n t}^{\varepsilon},
\end{gathered}
$$

where $\eta$ is a given positive constant and $\xi^{\varepsilon} \in H^{\frac{1}{2}}\left(\Gamma^{\varepsilon}\right)$. Using the transmission conditions on $\Gamma$ and the boundary condition on $\Gamma^{\varepsilon}$, the thin-layer transmission problem can be reduced to a uniquely solvable system of boundary integral equations of unknowns $\varphi, \psi$ and $\xi^{\varepsilon}$. For any distribution $\varphi \in H^{-\frac{1}{2}}(\Gamma)$, the potential 


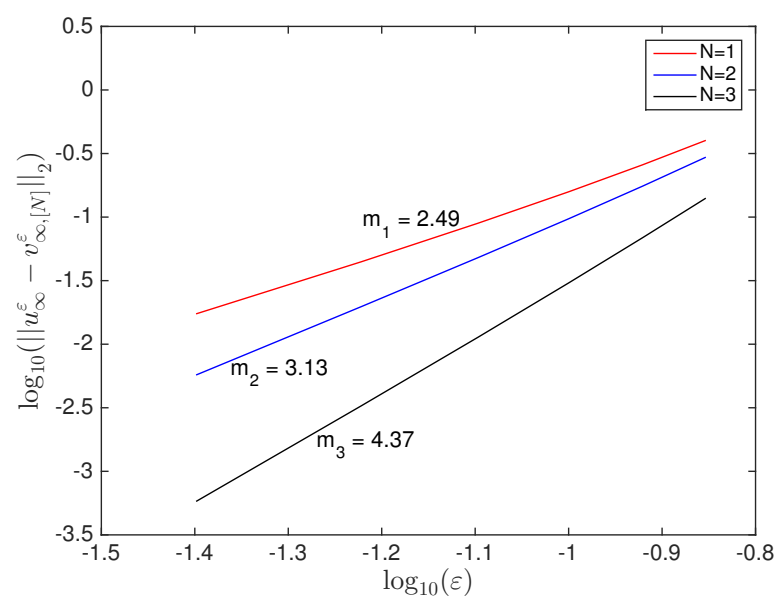

(a)

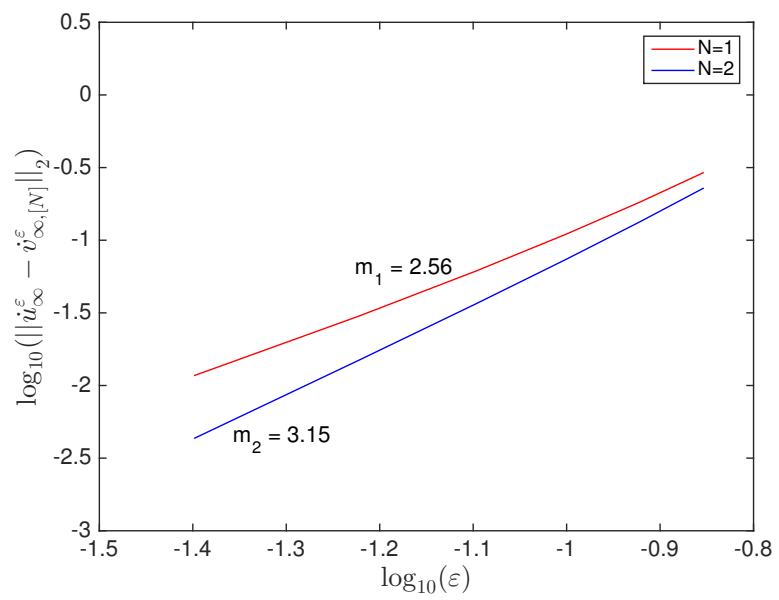

(c)

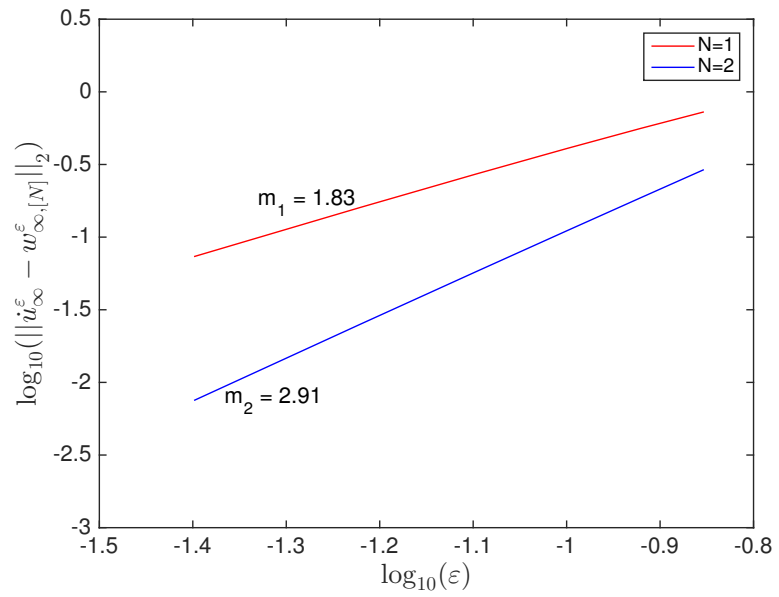

(e)

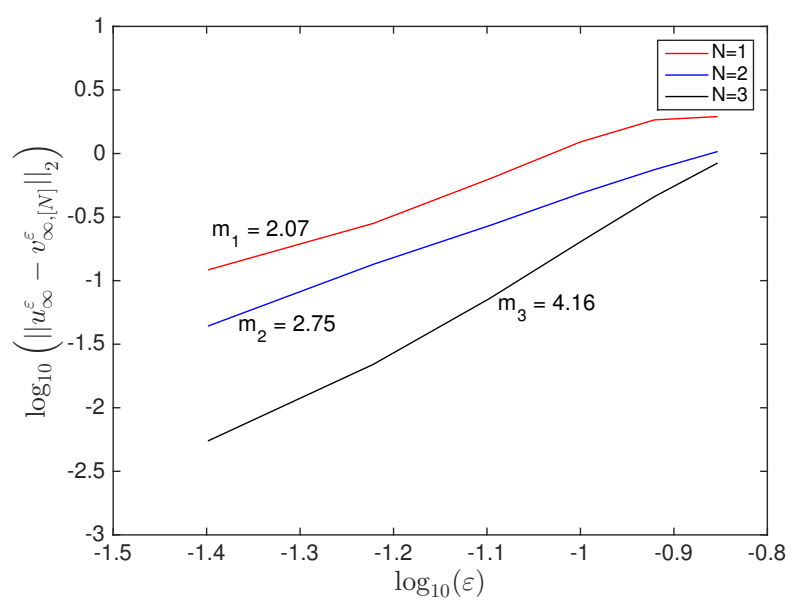

(b)

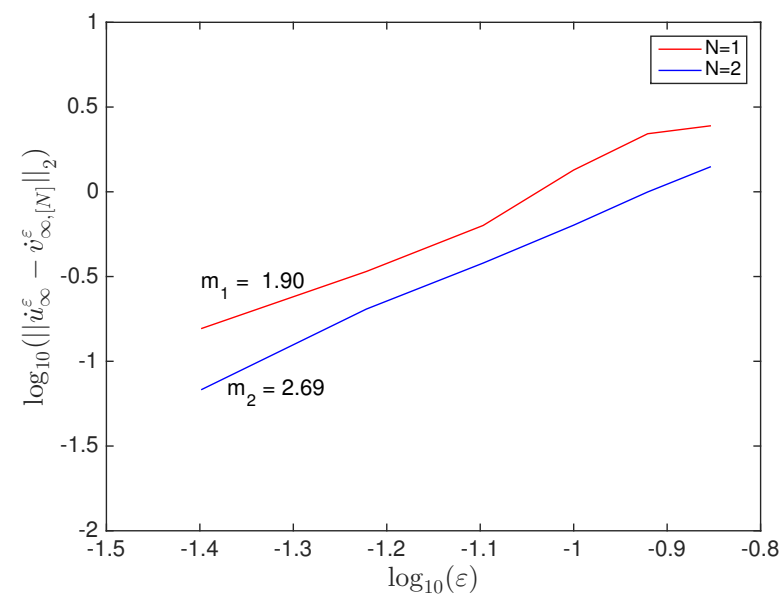

(d)

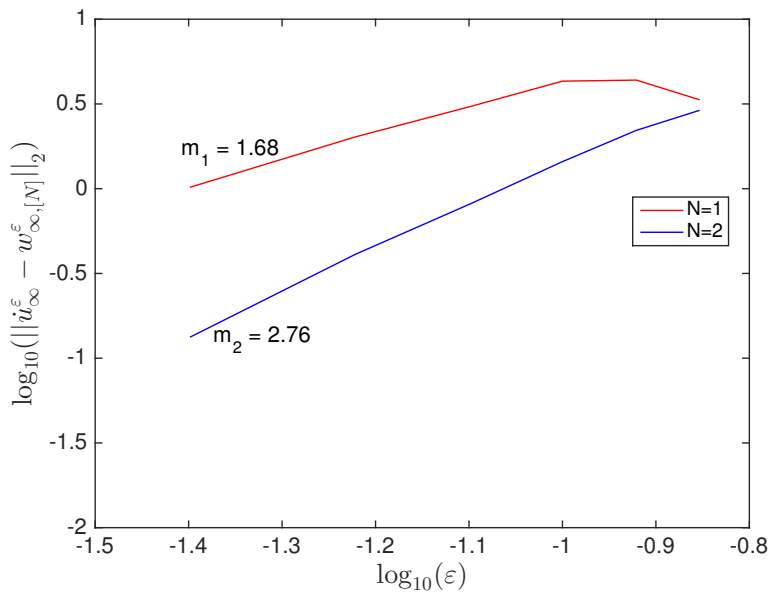

(f)

Figure 5: Numerical convergence, when $\varepsilon \rightarrow 0$, of the approximate far-field $v_{\infty,[N]}^{\varepsilon}$ to $u_{\infty}^{\varepsilon}$ and of the approximate shape derivatives $w_{\infty,[N]}^{\varepsilon}$ or $\dot{v}_{\infty,[N]}^{\varepsilon}$ to $\dot{u}_{\infty}^{\varepsilon}$ in the Dirichlet (left) and Neumann (right) cases.

$\mathcal{S}_{\Gamma}^{a} \varphi$ is analytical in any subdomain of $\mathbb{R}^{3} \backslash \Gamma$ and is continuous accross $\Gamma$. We have

$$
\left(\mathcal{S}_{\Gamma}^{a} \varphi\right)_{\mid \Gamma}=S_{\Gamma}^{a} \varphi \quad \text { and } \quad \lim _{s \rightarrow 0} \boldsymbol{n}(\boldsymbol{x}) \cdot\left(\nabla \mathcal{S}_{\Gamma}^{a} \varphi\right)(\boldsymbol{x} \pm s \boldsymbol{n}(\boldsymbol{x}))=\left(\mp \frac{1}{2} \mathrm{I}+D_{\Gamma}^{\prime a}\right) \varphi(\boldsymbol{x})
$$


where $\mathrm{I}$ is the identity operator and the involved boundary integral operators are defined for $\boldsymbol{x} \in \Gamma$ by

$$
\begin{aligned}
S_{\Gamma}^{a} \varphi(\boldsymbol{x}) & =\int_{\Gamma} G\left(\kappa_{a}, \boldsymbol{x}-\boldsymbol{y}\right) \varphi(\boldsymbol{y}) d \sigma(\boldsymbol{y}), \\
D_{\Gamma}^{\prime a} \varphi(\boldsymbol{x}) & =\int_{\Gamma} \partial_{\boldsymbol{n}}^{x} G\left(\kappa_{a}, \boldsymbol{x}-\boldsymbol{y}\right) \varphi(\boldsymbol{y}) d \sigma(\boldsymbol{y}) .
\end{aligned}
$$

For any distribution $\psi \in H^{\frac{1}{2}}(\Gamma)$, the potential $\mathcal{D}_{\Gamma}^{a} \varphi$ is analytical in any subdomain of $\mathbb{R}^{3} \backslash \Gamma$ and is discontinuous accross $\Gamma$. We have

$$
\lim _{s \rightarrow 0}\left(\mathcal{D}_{\Gamma}^{a} \psi\right)(\boldsymbol{x} \pm \operatorname{sn}(\boldsymbol{x}))=\left( \pm \frac{1}{2} \mathrm{I}+D_{\Gamma}^{a}\right) \psi(\boldsymbol{x}) \quad \text { and } \quad \partial_{\boldsymbol{n}} \mathcal{D}_{\Gamma}^{a} \psi=N_{\Gamma}^{a} \psi
$$

where the involved boundary integral operators are defined for $\boldsymbol{x} \in \Gamma$ by

$$
\begin{aligned}
D_{\Gamma}^{a} \psi(\boldsymbol{x}) & =\int_{\Gamma} \partial_{\boldsymbol{n}}^{\boldsymbol{y}} G\left(\kappa_{a}, \boldsymbol{x}-\boldsymbol{y}\right) \psi(\boldsymbol{y}) d \sigma(\boldsymbol{y}) \\
N_{\Gamma}^{a} \psi(\boldsymbol{x}) & =\int_{\Gamma} \partial_{\boldsymbol{n}}^{\boldsymbol{x}} \partial_{\boldsymbol{n}}^{\boldsymbol{y}} G\left(\kappa_{a}, \boldsymbol{x}-\boldsymbol{y}\right) \psi(\boldsymbol{y}) d \sigma(\boldsymbol{y}) .
\end{aligned}
$$

The operator $S_{\Gamma}^{a}$ is bounded from $\boldsymbol{H}^{-\frac{1}{2}}(\Gamma)$ to $\boldsymbol{H}^{\frac{1}{2}}(\Gamma)$ and compact from $\boldsymbol{H}^{-\frac{1}{2}}(\Gamma)$ to itself. The operators $D_{\Gamma}^{a}: \boldsymbol{H}^{\frac{1}{2}}(\Gamma) \rightarrow \boldsymbol{H}^{\frac{1}{2}}(\Gamma)$ and $D_{\Gamma}^{\prime a}: \boldsymbol{H}^{-\frac{1}{2}}(\Gamma) \rightarrow \boldsymbol{H}^{-\frac{1}{2}}(\Gamma)$ are compact and the operator $N_{\Gamma}^{a}: \boldsymbol{H}^{\frac{1}{2}}(\Gamma) \rightarrow$ $\boldsymbol{H}^{-\frac{1}{2}}(\Gamma)$ is bounded and has a hypersingular kernel. Using these results, we obtain the following system of second kind Fredholm boundary integral equations when a Dirichlet boundary condition is fulfilled on $\Gamma^{\varepsilon}$

$$
\left[\left(\begin{array}{ccc}
\frac{(1+\rho)}{2} \mathrm{I} & 0 & 0 \\
0 & \frac{(1+\rho)}{2} \mathrm{I} & 0 \\
0 & 0 & \frac{1}{2} \mathrm{I}
\end{array}\right)+\left(\begin{array}{ccc}
\rho D_{\Gamma}^{e}-D_{\Gamma}^{i} & S_{\Gamma}^{i}-S_{\Gamma}^{e} & -\left(\left(\mathcal{D}_{\Gamma^{\varepsilon}}^{i}\right)_{\mid \Gamma}+i \eta\left(\mathcal{S}_{\Gamma^{\varepsilon}}^{i}\right)_{\mid \Gamma}\right) \\
\rho\left(N_{\Gamma}^{e}-N_{\Gamma}^{i}\right) & \rho D_{\Gamma}^{\prime i}-D_{\Gamma}^{\prime e} & -\rho\left(\left(\partial_{\boldsymbol{n}} \mathcal{D}_{\Gamma^{\varepsilon}}^{i}\right)_{\mid \Gamma}+i \eta\left(\partial_{\boldsymbol{n}} \mathcal{S}_{\Gamma^{\varepsilon}}^{i}\right)_{\mid \Gamma}\right) \\
\left(\mathcal{D}_{\Gamma}^{i}\right)_{\mid \Gamma^{\varepsilon}} & -\left(\mathcal{S}_{\Gamma^{i}}^{i}\right)_{\Gamma^{\varepsilon}} & D_{\Gamma^{\varepsilon}}^{i}+i \eta S_{\Gamma^{\varepsilon}}^{i}
\end{array}\right)\right]\left(\begin{array}{c}
\psi \\
\varphi \\
\xi^{\varepsilon}
\end{array}\right)=\left(\begin{array}{l}
-f_{\text {ext }} \\
-g_{\text {ext }} \\
-f_{\text {int }}^{\varepsilon}
\end{array}\right) .
$$

The associated integral equation operator is invertible from $H^{\frac{1}{2}}(\Gamma) \times H^{-\frac{1}{2}}(\Gamma) \times H^{\frac{1}{2}}\left(\Gamma^{\varepsilon}\right)$ to itself. When a Neumann boundary condition is fulfilled on $\Gamma^{\varepsilon}$, we obtain the following system of boundary integral equations

$$
\left[\left(\begin{array}{ccc}
\frac{(1+\rho)}{2} \mathrm{I} & 0 & 0 \\
0 & \frac{(1+\rho)}{2} \mathrm{I} & 0 \\
0 & 0 & N_{\Gamma^{\varepsilon}}^{i}
\end{array}\right)+\left(\begin{array}{ccc}
\rho D_{\Gamma}^{e}-D_{\Gamma}^{i} & S_{\Gamma}^{i}-S_{\Gamma}^{e} & -\left(\left(\mathcal{D}_{\Gamma^{\varepsilon}}^{i}\right)_{\mid \Gamma}+i \eta\left(\mathcal{S}_{\Gamma^{\varepsilon}}^{i}\right)_{\mid \Gamma}\right) \\
\rho\left(N_{e}-N_{i}\right) & \rho D_{\Gamma}^{\prime i}-D_{\Gamma}^{\prime e} & -\rho\left(\left(\partial_{\boldsymbol{n}} \mathcal{D}_{\Gamma^{\varepsilon}}^{i}\right)_{\mid \Gamma}+i \eta\left(\partial_{\boldsymbol{n}} \mathcal{S}_{\Gamma^{\varepsilon}}^{i}\right)_{\mid \Gamma}\right) \\
\left(\partial_{\boldsymbol{n}_{\varepsilon}} \mathcal{D}_{\Gamma}^{i}\right)_{\mid \Gamma^{\varepsilon}} & -\left(\partial_{\boldsymbol{n}_{\varepsilon}} \mathcal{S}_{\Gamma}^{i}\right)_{\mid \Gamma^{\varepsilon}} & i \eta D_{\Gamma^{\varepsilon}}^{\prime i}-\frac{i \eta}{2} \mathrm{I}
\end{array}\right)\right]\left(\begin{array}{c}
\psi \\
\varphi \\
\xi^{\varepsilon}
\end{array}\right)=\left(\begin{array}{c}
-f_{\text {ext }} \\
-g_{\text {ext }} \\
-g_{\text {int }}^{\varepsilon}
\end{array}\right) .
$$

Using regularization technique for the third equation, we can prove that the associated integral equation operator is invertible from $H^{\frac{1}{2}}(\Gamma) \times H^{-\frac{1}{2}}(\Gamma) \times H^{\frac{1}{2}}\left(\Gamma^{\varepsilon}\right)$ to $H^{\frac{1}{2}}(\Gamma) \times H^{-\frac{1}{2}}(\Gamma) \times H^{-\frac{1}{2}}\left(\Gamma^{\varepsilon}\right)$. The farfield pattern associated to the exterior field is then given by

$$
u_{\infty}^{\varepsilon}=\rho \mathcal{D}_{\Gamma}^{\infty} \psi-\mathcal{S}_{\Gamma}^{\infty} \varphi, \quad \text { on } \mathbb{S}^{2},
$$

with

$$
\begin{gathered}
\mathcal{D}_{\Gamma}^{\infty} \psi(\hat{\boldsymbol{x}})=-\frac{i \kappa_{e}}{4 \pi} \int_{\Gamma}(\hat{\boldsymbol{x}} \cdot \boldsymbol{n}(\boldsymbol{y})) e^{-i \kappa_{e} \hat{\boldsymbol{x}} \cdot \boldsymbol{y}} \psi(\boldsymbol{y}) d s(\boldsymbol{y}), \\
\mathcal{S}_{\Gamma}^{\infty} \varphi(\hat{\boldsymbol{x}})=\frac{1}{4 \pi} \int_{\Gamma} e^{-i \kappa_{e} \hat{\boldsymbol{x}} \cdot \boldsymbol{y}} \varphi(\boldsymbol{y}) d s(\boldsymbol{y}) .
\end{gathered}
$$

The direct approached is used when $f_{\text {ext }}$ and $g_{\text {ext }}$ are the boundary data of the time-harmonic incident plane wave $u^{i n c}$ and $f_{i n t}^{\varepsilon}=0=g_{i n t}^{\varepsilon}$. Then we have the following integral representation

$$
\begin{gathered}
u_{\text {ext }}^{\varepsilon}=\mathcal{D}_{\Gamma}^{e}\left(u_{\text {ext }}^{\varepsilon}+u^{i n c}\right)-\mathcal{S}_{\Gamma}^{e} \partial_{\boldsymbol{n}}\left(u_{\text {ext }}^{\varepsilon}+u^{i n c}\right), \quad \text { in } \Omega_{\text {ext }}, \\
u_{i n t}^{\varepsilon}=\left(-\mathcal{D}_{\Gamma}^{i}\left(u_{\text {ext }}^{\varepsilon}+u^{i n c}\right)+\rho \mathcal{S}_{\Gamma}^{i} \partial_{\boldsymbol{n}}\left(u_{\text {ext }}^{\varepsilon}+u^{i n c}\right)\right)+\left(\mathcal{D}_{\Gamma^{\varepsilon}}^{i} u_{i n t}^{\varepsilon}-\mathcal{S}_{\Gamma^{\varepsilon}}^{i} \partial_{\boldsymbol{n}} u_{i n t}^{\varepsilon}\right), \quad \text { in } \Omega_{\text {int }}^{\varepsilon},
\end{gathered}
$$

The transmission conditions and the Dirichlet condition $u_{i n t}^{\varepsilon}=0$ yield

$\left[\left(\begin{array}{l}\frac{(1+\rho)}{2} \mathrm{I} \\ 0 \\ 0\end{array}\right.\right.$

$\left.\begin{array}{cc}0 & 0 \\ \frac{(1+\rho)}{2} \mathrm{I} & 0 \\ 0 & \frac{1}{2} \mathrm{I}\end{array}\right)+\left(\begin{array}{cc}\rho D_{\Gamma}^{i}-D_{\Gamma}^{e} & S_{\Gamma}^{e}-S_{\Gamma}^{i} \\ \rho\left(N_{\Gamma}^{i}-N_{\Gamma}^{e}\right) & \rho D_{\Gamma}^{\prime e}-D_{\Gamma}^{\prime i} \\ -\left(\left(\mathcal{D}_{\Gamma}^{i}\right)_{\mid \Gamma^{\varepsilon}}+i \eta \partial_{\boldsymbol{n}^{\varepsilon}}\left(\mathcal{D}_{\Gamma}^{i}\right)_{\mid \Gamma^{\varepsilon}}\right) & \frac{1}{\rho}\left(\left(\mathcal{D}_{\Gamma}^{i}\right)_{\mid \Gamma^{\varepsilon}}+i \eta \partial_{\boldsymbol{n}^{\varepsilon}}\left(\mathcal{D}_{\Gamma}^{i}\right)_{\mid \Gamma^{\varepsilon}}\right)\end{array}\right.$ $\left.\left.\begin{array}{c}-\rho\left(\mathcal{S}_{\Gamma^{\varepsilon}}^{i}\right)_{\mid \Gamma} \\ -\rho\left(\partial_{\boldsymbol{n}} \mathcal{S}_{\Gamma^{\varepsilon}}^{i}\right)_{\mid \Gamma} \\ D_{\Gamma^{\varepsilon}}^{\prime i}+i \eta S_{\Gamma^{\varepsilon}}^{i}\end{array}\right)\right]\left(\begin{array}{c}\left(u_{\text {ext }}^{\varepsilon}+u^{i n c}\right) \\ \partial_{\boldsymbol{n}}\left(u_{\text {ext }}^{\varepsilon}+u^{i n c}\right) \\ u_{\text {int }}^{\varepsilon}\end{array}\right)=\left(\begin{array}{c}u^{i n c} \\ \rho \partial_{\boldsymbol{n}} u^{i n c} \\ 0\end{array}\right)$ 
The transmission conditions and the Neumann condition $\partial_{\boldsymbol{n}} u_{i n t}^{\varepsilon}=0$ yield

$$
\left[\left(\begin{array}{ccc}
\frac{(1+\rho)}{2} \mathrm{I} & 0 & 0 \\
0 & \frac{(1+\rho)}{2} \mathrm{I} & 0 \\
0 & 0 & N_{\Gamma^{\varepsilon}}^{i}
\end{array}\right)+\left(\begin{array}{ccc}
\rho D_{\Gamma}^{i}-D_{\Gamma}^{e} & S_{\Gamma}^{e}-S_{\Gamma}^{i} & \rho\left(\mathcal{D}_{\Gamma^{\varepsilon}}^{i}\right)_{\mid \Gamma} \\
\rho\left(N_{i}-N_{e}\right) & \rho D_{\Gamma}^{\prime e}-D_{\Gamma}^{\prime i} & \rho\left(\partial_{\boldsymbol{n}} \mathcal{D}_{\Gamma^{\varepsilon}}^{i}\right)_{\mid \Gamma} \\
-\left(\left(\mathcal{D}_{\Gamma}^{i}\right)_{\mid \Gamma^{\varepsilon}}+i \eta \partial_{\boldsymbol{n}^{\varepsilon}}\left(\mathcal{D}_{\Gamma}^{i}\right)_{\mid \Gamma^{\varepsilon}}\right) & \frac{1}{\rho}\left(\left(\mathcal{D}_{\Gamma}^{i}\right)_{\mid \Gamma^{\varepsilon}}+i \eta \partial_{\boldsymbol{n}^{\varepsilon}}\left(\mathcal{D}_{\Gamma}^{i}\right)_{\mid \Gamma^{\varepsilon}}\right) & i \eta D_{\Gamma^{\varepsilon}}^{i}-\frac{i \eta}{2} \mathrm{I}
\end{array}\right)\right]\left(\begin{array}{c}
\left(u_{\text {ext }}^{\varepsilon}+u^{i n c}\right) \\
\partial \boldsymbol{n}\left(u_{e x t}^{\varepsilon}+u^{i n c}\right) \\
\partial_{\boldsymbol{n}^{\varepsilon}} u_{i n t}^{\varepsilon}
\end{array}\right)=\left(\begin{array}{c}
u^{i n c} \\
\rho \partial_{\boldsymbol{n}} u^{i n c} \\
0
\end{array}\right) .
$$

The farfield pattern associated to the exterior field is then given by

$$
u_{\infty}^{\varepsilon}=\mathcal{D}_{\Gamma}^{\infty}\left(u_{e x t}^{\varepsilon}+u^{i n c}\right)-\mathcal{S}_{\Gamma}^{\infty} \partial_{\boldsymbol{n}}\left(u_{\text {ext }}^{\varepsilon}+u^{i n c}\right), \quad \text { on } \mathbb{S}^{2},
$$

The direct method is used to compute the solution to the forward problem while the indirect one is required to compute the shape derivatives of the solution. The direct method has the advantage to provide the boundary data which are needed to compute the boundary data of the shape derivatives (see Theorem 4.1).

The exterior problems (3.1) can be solved, for $N=1,2,3$, using boundary integral equation methods. Here again we consider two approaches. The indirect one is based on the layer ansatz

$$
v_{[N]}^{\varepsilon}=\mathcal{S}_{\Gamma}^{e} \varphi \quad \text { in } \Omega_{e x t}
$$

and can be used for solving the exterior problems 3.1 when the boundary condition is written in the form $C\left(\varepsilon, \partial_{\boldsymbol{n}}\left(v_{[N]}^{\varepsilon}\right),\left(v_{[N]}^{\varepsilon}\right)\right)=F$. The exterior problem can be reduced to the following boundary integral equation for the unknown $\varphi$

$$
C\left(\varepsilon,-\frac{1}{2} \mathrm{I}+D_{\Gamma}^{\prime e}, S_{\Gamma}^{e}\right) \varphi=-C\left(\varepsilon, \partial_{n} u^{i n c}, u^{i n c}\right) .
$$

The farfield pattern associated to the exterior field is then given by $v_{\infty,[N]}^{\varepsilon}=\mathcal{S}_{\Gamma}^{\infty} \varphi$

The direct one is based on the integral representation formula of the exterior wave

$$
v_{[N]}^{\varepsilon}=\mathcal{D}_{\Gamma}^{e}\left(v_{[N]}^{\varepsilon}+u^{i n c}\right)-\mathcal{S}_{\Gamma}^{e} \partial_{\boldsymbol{n}}\left(v_{[N]}^{\varepsilon}+u^{i n c}\right), \quad \text { in } \Omega_{e x t},
$$

In the case of a Dirichlet boundary condition, the GIBCs given in Theorem 3.1 yield the boundary integral equation

$$
\left[\left(\frac{1}{2} \mathrm{I}-D_{\Gamma}^{e}\right)\left(-B^{\varepsilon, N}\right)-S_{\Gamma}^{e}\right] \partial_{n}\left(v_{[N]}^{\varepsilon}+u^{i n c}\right)=u^{i n c} .
$$

We obtain the other boundary data by computing $\left(v_{[N]}^{\varepsilon}+u^{i n c}\right)=-B^{\varepsilon, N} \partial_{n}\left(v_{[N]}^{\varepsilon}+u^{i n c}\right)$. In the case of a Neumann boundary condition, the GIBCs given in Theorem 3.1 yield the boundary integral equation

$$
\left[\left(\frac{1}{2} \mathrm{I}-D_{\Gamma}^{e}\right)-S_{\Gamma}^{e}\left(-B^{\varepsilon, N}\right)\right]\left(v_{[N]}^{\varepsilon}+u^{i n c}\right)=u^{i n c} .
$$

We obtain the other boundary data by computing $\partial_{\boldsymbol{n}}\left(v_{[N]}^{\varepsilon}+u^{i n c}\right)=-B^{\varepsilon, N}\left(v_{[N]}^{\varepsilon}+u^{i n c}\right)$. The farfield pattern associated to the exterior field is then given by A.6.

Here again, the direct method is used to compute the solution to the forward problem while the indirect one is required to compute the shape derivatives of the solution. The direct method has the advantage to provide the boundary data which are needed to compute the boundary data of the shape derivatives. The integral formulation may suffer from irregular frequencies, however we consider wavenumbers out of the discrete set of eigenvalues.

\section{Acknowledgment}

The authors gratefully acknowledge Prof. Marc Dambrine from Université de Pau et des Pays de l'Adour, Laboratory IPRA-LMAP, for suggesting the subject of this paper.

\section{References}

[1] L. Afraites, M. Dambrine, And D. Kateb, On second order shape optimization methods for electrical impedance tomography, SIAM J. Control Optim., 47 (2008), pp. 1556-1590.

[2] H. Ammari And J.-C. NÉDÉLEC, Generalized impedance boundary conditions for the Maxwell equations as singular perturbations problems, Comm. Partial Differential Equations, 24 (1999), pp. 821-849.

[3] B. Aslanyürek, H. Haddar, And H. ŞAhintürk, Generalized impedance boundary conditions for thin dielectric coatings with variable thickness, Wave Motion, 48 (2011), pp. 681-700. 
[4] A. Bendali and K. Lemrabet, The effect of a thin coating on the scattering of a time-harmonic wave for the Helmholtz equation, SIAM J. Appl. Math., 56 (1996), pp. 1664-1693.

[5] - Asymptotic analysis of the scattering of a time-harmonic electromagnetic wave by a perfectly conducting metal coated with a thin dielectric shell, Asymptot. Anal., 57 (2008), pp. 199-227.

[6] M. Bonnet, A. Burel, M. Duruflé, And P. Joly, Effective transmission conditions for thin-layer transmission problems in elastodynamics. The case of a planar layer model, ESAIM: Mathematical Modelling and Numerical Analysis, 50 (2016), pp. 43-75.

[7] L. Bourgeois, N. Chaulet, and H. Haddar, Stable reconstruction of generalized impedance boundary conditions, Inverse Problems, 27 (2011), pp. 095002, 26.

[8] — On simultaneous identification of the shape and generalized impedance boundary condition in obstacle scattering, SIAM J. Sci. Comput., 34 (2012), pp. A1824-A1848.

[9] F. CAKONI AND R. KREss, Integral equation methods for the inverse obstacle problem with generalized impedance boundary condition, Inverse Problems, 29 (2013), pp. 015005, 19.

[10] G. Caloz, M. Costabel, M. Dauge, and G. Vial, Asymptotic expansion of the solution of an interface problem in a polygonal domain with thin layer, Asymptot. Anal., 50 (2006), pp. 121-173.

[11] F. Caubet, M. Dambrine, And D. Kateb, Shape optimization methods for the inverse obstacle problem with generalized impedance boundary conditions, Inverse Problems, 29 (2013), pp. 115011, 26.

[12] N. Chaulet, Modèles d'impédance généralisée en diffraction inverse, PhD thesis, École Polytechnique, 2012.

[13] David Colton and Rainer Kress, Inverse acoustic and electromagnetic scattering theory, vol. 93 of Applied Mathematical Sciences, Springer, New York, third ed., 2013.

[14] M. Costabel And F. Le Louër, Shape derivatives of boundary integral operators in electromagnetic scattering. Part I: Shape differentiability of pseudo-homogeneous boundary integral operators, Integral Equations and Operator Theory, 72 (2012), pp. 509-535.

[15] M. Dambrine, D. Kateb, and J. Lamboley, An extremal eigenvalue problem for the WentzellLaplace operator, Annales de l'Institut Henri Poincaré (C) Non Linear Analysis, (2015), p. electronic.

[16] F. Desaint And J.-P. ZolÉsio, Manifold derivative in the laplace-beltrami equation, Journal of Functional Analysis, 151 (1997), pp. $234-269$.

[17] M. Duruflé, H. Haddar, And P. Joly, High Order Generalized Impedance Boundary Conditions in Electromagnetic Scattering Problems, Comptes Rendus de l'Academie des Sciences de Paris Serie Physique, 7 (2006), pp. 533-542.

[18] M. Ganesh and I. G. Graham, A high-order algorithm for obstacle scattering in three dimensions, J. Comput. Phys., 198 (2004), pp. 211-242.

[19] H. Haddar And P. Joly, Stability of thin layer approximation of electromagnetic waves scattering by linear and nonlinear coatings, J. Comput. Appl. Math., 143 (2002), pp. 201-236.

[20] F. Heтtlich, Fréchet derivatives in inverse obstacle scattering, Inverse Problems, 11 (1995), pp. 371382.

[21] _ Erratum: "Frechet derivatives in inverse obstacle scattering" [Inverse Problems 11 (1995), no. 2, 371-382; MR1324650 (95k:35217)], Inverse Problems, 14 (1998), pp. 209-210.

[22] _ Iterative Methods in Inverse Obstacle Scattering: Regularization Theory of Linear and Nonlinear Exponentially Ill-Posed Problems, PhD thesis, University of Linz, 1999.

[23] Thorsten Hohage and Frédérique Le Louër, A spectrally accurate method for the dielectric obstacle scattering problem and applications to the inverse problem, preprint, (2013). http://num.math.uni-goettingen.de/preprints/files/2013-20.pdf.

[24] T. Hohage and C. Schormann, A Newton-type method for a transmission problem in inverse scattering, Inverse Problems, 14 (1998), pp. 1207-1227.

[25] R. Kress, F. Yaman, A. Yapar, and I. Akduman, Inverse scattering for an impedance cylinder buried in a dielectric cylinder, Inverse Probl. Sci. Eng., 17 (2009), pp. 473-488.

[26] K. Lemrabet, Le problème de Ventcel pour le système de l'élasticité dans un domaine de $\mathbf{R}^{3}, \mathrm{C} . \mathbf{R}$. Acad. Sci. Paris Sér. I Math., 304 (1987), pp. 151-154.

[27] J.-C. NÉdÉLEc, Acoustic and electromagnetic equations, vol. 144 of Applied Mathematical Sciences, Springer-Verlag, New York, 2001. Integral representations for harmonic problems. 
[28] M. Pierre and A. Henrot, Variation et Optimisation de Formes, une Analyse géométrique, Springer, 2005.

[29] V. PÉRon, Equivalent boundary conditions for an elasto-acoustic problem set in a domain with a thin layer, ESAIM Math. Model. Numer. Anal., 48 (2014), pp. 1431-1449.

[30] R. Potthast, Fréchet differentiability of boundary integral operators in inverse acoustic scattering, Inverse Problems, 10 (1994), pp. 431-447.

[31] _ Fréchet differentiability of the solution to the acoustic Neumann scattering problem with respect to the domain, J. Inverse Ill-Posed Probl., 4 (1996), pp. 67-84.

[32] J. L. VÁzQuez and E. Vitillaro, On the Laplace equation with dynamical boundary conditions of reactive-diffusive type, J. Math. Anal. Appl., 354 (2009), pp. 674-688.

[33] G. VIAL, Analyse multi-échelle et conditions aux limites approchées pour un problème avec couche mince dans un domaine à coin, $\mathrm{PhD}$ thesis, Université de Rennes 1, 2003.

[34] F. YAMAN, Location and shape reconstructions of sound-soft obstacles buried in penetrable cylinders, Inverse Problems, 25 (2009), pp. 065005, 17. 